\title{
RiqueZa y DistribuCión de la familia SOlanaCEAe en el estado de Aguascalientes, México
}

\author{
José Carlos Sierra-Muñoz', María Elena Siqueiros-Delgado, Ernesto Flores-AnCira, \\ Onésimo Moreno-Rico y José Luis Arredondo-Figueroa \\ Herbario HUAA, Departamento de Biología, Centro de Ciencias Básicas, Universidad Autónoma de Aguascalientes, \\ Aguascalientes, Aguascalientes, México \\ 1Autor para la correspondencia: jcsierra@correo.uaa.mx
}

\begin{abstract}
Resumen: Solanaceae es una de las familias económicamente más importantes a nivel mundial y México es reconocido como uno de sus centros de diversificación. Sin embargo, los trabajos taxonómicos y los reportes de diversidad generados en distintos estados de la República no son suficientes para tener una visión general acerca del número de especies y su distribución en el territorio nacional. El objetivo del presente estudio fue contribuir al conocimiento de la familia Solanaceae en México, dando a conocer la riqueza y distribución de las especies que se desarrollan en el estado de Aguascalientes. Los datos se obtuvieron de colectas y del análisis de 862 ejemplares de herbario. La familia Solanaceae en Aguascalientes está representada por 55 especies (16 nuevos registros) agrupadas en 12 géneros. Los géneros mejor representados son Solanum (23 especies) y Physalis (14), seguidos por Nicotiana y Datura (4), Bouchetia y Lycianthes (2), y Cestrum, Chamaesaracha, Jaltomata, Nicandra, Nierembergia y Petunia están representados por una especie cada uno. Solanaceae presenta una amplia distribución en el estado, desde los 1,600 a los 3,000 m de altitud. Está presente en todas las comunidades vegetales, destacan el matorral semiárido (32 especies), matorral subtropical (30), bosque de encino (28), la vegetación hidrófila (21) y el pastizal desértico (20). Gran cantidad de especies se desarrollan como malezas, por lo que es común encontrarlas en áreas con diversos grados de disturbio. Solanaceae representa la quinta familia más rica en especies del estado de Aguascalientes.
\end{abstract}

Palabras clave: distribución, flora, matorral semiárido, México, riqueza.

\begin{abstract}
Solanaceae is one of the most economically important families worldwide. Mexico is recognized as a center of diversification. However, so far we do not have an overview on the current state of the family in the country. The aim of this study was to contribute to the knowledge of the Solanaceae in Mexico, by providing data on the richness and distribution of the species growing in the state of Aguascalientes. Derived of the study of 862 herbarium specimens and collections of Solanaceae from Aguascalientes, 55 species ( 16 new records) in 12 genera were recorded. The largest genera are Solanum ( 23 species) and Physalis (14), followed by Nicotiana and Datura (4), Bouchetia and Lycianthes (2), and Cestrum, Chamaesaracha, Jaltomata, Nicandra, Nierembergia, and Petunia with one species each. Solanaceae is widely distributed in the state from 1,600 to 3,000 m altitude. It is present in all plant communities, highlighting the semiarid scrub (32 species), subtropical scrub (30), oak forest (28), hydrophilic vegetation (21) and desert grassland (20). Many species grow as weeds, so they are commonly found in perturbed areas. Solanaceae represents the fifth family with more richness of species in the state of Aguascalientes.
\end{abstract}

Key words: distribution, flora, semiarid scrub, Mexico, richness.

$\mathbf{L}^{2}$ a familia Solanaceae se encuentra asignada al orden Solanales, junto con Convolvulaceae, Hydroleaceae, Montiniaceae y Sphenocleaceae (Eckart, 2008). Contiene aproximadamente 96 géneros y 2,300 especies (CuevasArias et al., 2008). Presenta distribución cosmopolita, con mayor frecuencia en regiones tropicales, subtropicales y templadas, en un intervalo altitudinal de 0 a 4,000 m. Tiene gran variedad de formas vegetativas y reproductivas, por lo que tiene la capacidad de colonizar distintos tipos de hábitats. Se caracteriza por presentar flores hermafroditas y actinomorfas, en general pentámeras y con gran variación en tamaño; ovario súpero, esencialmente bilocular y fruto generalmente en forma de una baya o cápsula. También es notable por producir, en la mayoría de sus órganos, alca- 
loides tóxicos como la nicotina, la atropina y la solanaína; además de ciertos esteroides conocidos como glicoalcaloides, usados como precursores de hormonas (Nee, 1986). Asimismo, incluye especies de gran importancia: alimenticia (papa, jitomate, tomate, berenjena y chile); industrial (tabaco); ornamental (petunias, floripondio, copa de oro y huele de noche) y medicinal (toloache y belladona).

De acuerdo a Cuevas-Arias et al. (2008), los géneros con mayor número de especies son: Solanum $(1,000)$, Lycianthes (200), Cestrum (175), Nicotiana (95), Physalis (80) y Lycium (75). Aunque la mayoría de los especialistas en la familia opinan que su centro de origen se ubica en América del Sur (Long, 2001), algunos de sus géneros se desarrollaron ampliamente en otros países, de manera que actualmente se mencionan varios centros de diversificación, entre ellos Australia, los Himalaya y México.

Debido a su ubicación geográfica, compleja historia geológica y combinación de condiciones climáticas, México es el tercer país con mayor diversidad biológica del mundo (Toledo y Ordóñez, 1993) y su riqueza florística se estima en alrededor de 26,500 especies (Encina-Domínguez et al., 2007). Sin embargo, a pesar de su importancia biológica, económica y cultural, se conoce poco la diversidad y distribución de varias familias que habitan en el territorio nacional; entre ellas Solanaceae, debido probablemente a la complejidad morfológica del grupo, al número de especies que contiene y a la diversidad de hábitats que coloniza. Aunado a que los trabajos taxonómicos y los reportes de diversidad que se han generado en diversos estados de la República no son suficientes para tener una visión general en torno a la riqueza y distribución que presenta la familia Solanaceae en México.

En el estado de Aguascalientes se han realizado diversos estudios florísticos (de la Cerda, 1982, 1996, 1999a, b, 2004, 2011; García, 1998, 2004, 2005; González-Adame, 2004; Siqueiros, 1996, 1999; Siqueiros y González, 2006; Siqueiros et al., 2011), los cuales ubican a la familia Solanaceae en el cuarto lugar en cuanto al número de especies, después de Asteraceae, Poaceae y Fabaceae. Sin embargo, hasta la fecha no se ha llevado a cabo un estudio exhaustivo de la familia Solanaceae en el estado de Aguascalientes.

Por ello, el objetivo del presente estudio fue contribuir al conocimiento de la familia Solanaceae en México, dando a conocer la riqueza y distribución de las especies que se desarrollan en el estado de Aguascalientes.

\section{Materiales y métodos}

Región de estudio. El estado de Aguascalientes se localiza en el centro de México (Figura 1), en la región geográfica del Altiplano Mexicano. Comprende una superficie aproximada de $5,471 \mathrm{~km}^{2}$ y se ubica entre los $21^{\circ} 38^{\prime} 03^{\prime \prime}$ y $22^{\circ}$ 27 ' $06^{\prime}$ " de latitud norte y entre los $101^{\circ} 53^{\prime} 09^{\prime}$ ' y los $103^{\circ}$ 00' 51 " de longitud oeste. Limita al norte, este y oeste con el estado de Zacatecas y al sur con el estado de Jalisco (de la Cerda, 2011). La división política consta de once municipios: Aguascalientes, Asientos, Calvillo, Cosío, El Llano, Jesús María, Pabellón de Arteaga, Rincón de Romos, San Francisco de los Romo, San José de Gracia y Tepezalá (Figura 1). Está ubicado entre tres provincias geológicas: Sierra Madre Occidental, Mesa Central y Faja Volcánica Transmexicana, con elevaciones que van de los 1,540 a los 3,050 m. Las rocas que predominan en la entidad son las ígneas extrusivas ácidas: riolitas y tobas. Le siguen en importancia las rocas sedimentarias de origen continental: areniscas y conglomerados del Terciario. En el territorio existen trece de los 25 tipos de suelos reconocidos a nivel mundial. Los más importantes por su extensión son los Feozems, Litosoles, Planosoles y Xerosoles, que en conjunto abarcan casi el $80 \%$ de la superficie estatal (CONABIO, 2008). Queda comprendido dentro de dos regiones hidrológicas: Lerma-Chapala-Santiago, que abarca la mayor parte del estado, y El Salado, constituido por una serie de cuencas cerradas de diferentes dimensiones (de la Cerda, 2011). En general el clima puede considerarse dentro del grupo de los semisecos (BS), con varios subtipos: semiseco templado $(\mathrm{BS} 1 \mathrm{Kw}(\mathrm{w}))$ y semiseco semicálido (BS-1hw), con una temperatura media anual que fluctúa entre 18 y $20^{\circ} \mathrm{C}$, y una precipitación media de $526 \mathrm{~mm}$, con un régimen de lluvias en verano (Franco et al., 2012).

La vegetación en el estado de Aguascalientes presenta aproximadamente un $38 \%$ de condición primaria y el restante $62 \%$ se considera en condición secundaria. De acuerdo a Rodríguez-Ávalos (2014), en el estado se pueden encontrar diez tipos de vegetación con diferentes grados de perturbación (Figura 2). (1) Bosque de encino: constituye el tipo de bosque más extendido en el estado, se localiza principalmente en laderas bajas, al pie de las sierras, y se ubica entre los 1,800 y 2,500 m de altitud. (2) Bosque de coníferas: se caracteriza por la presencia de pinos (Pinus spp.), ciprés

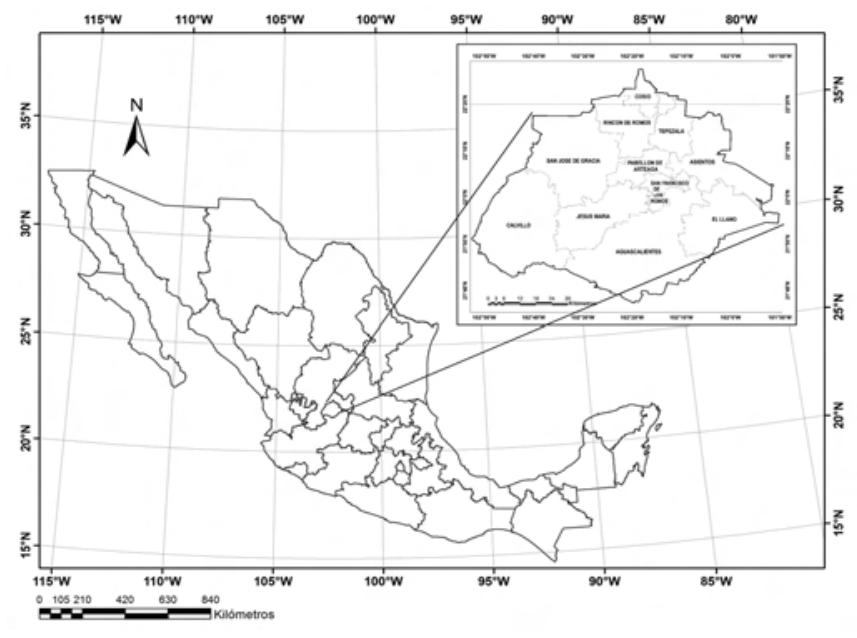

Figura 1. Ubicación geográfica y división política del estado de Aguascalientes.

Botanical Sciences 93 (1): 97-117, 2015 


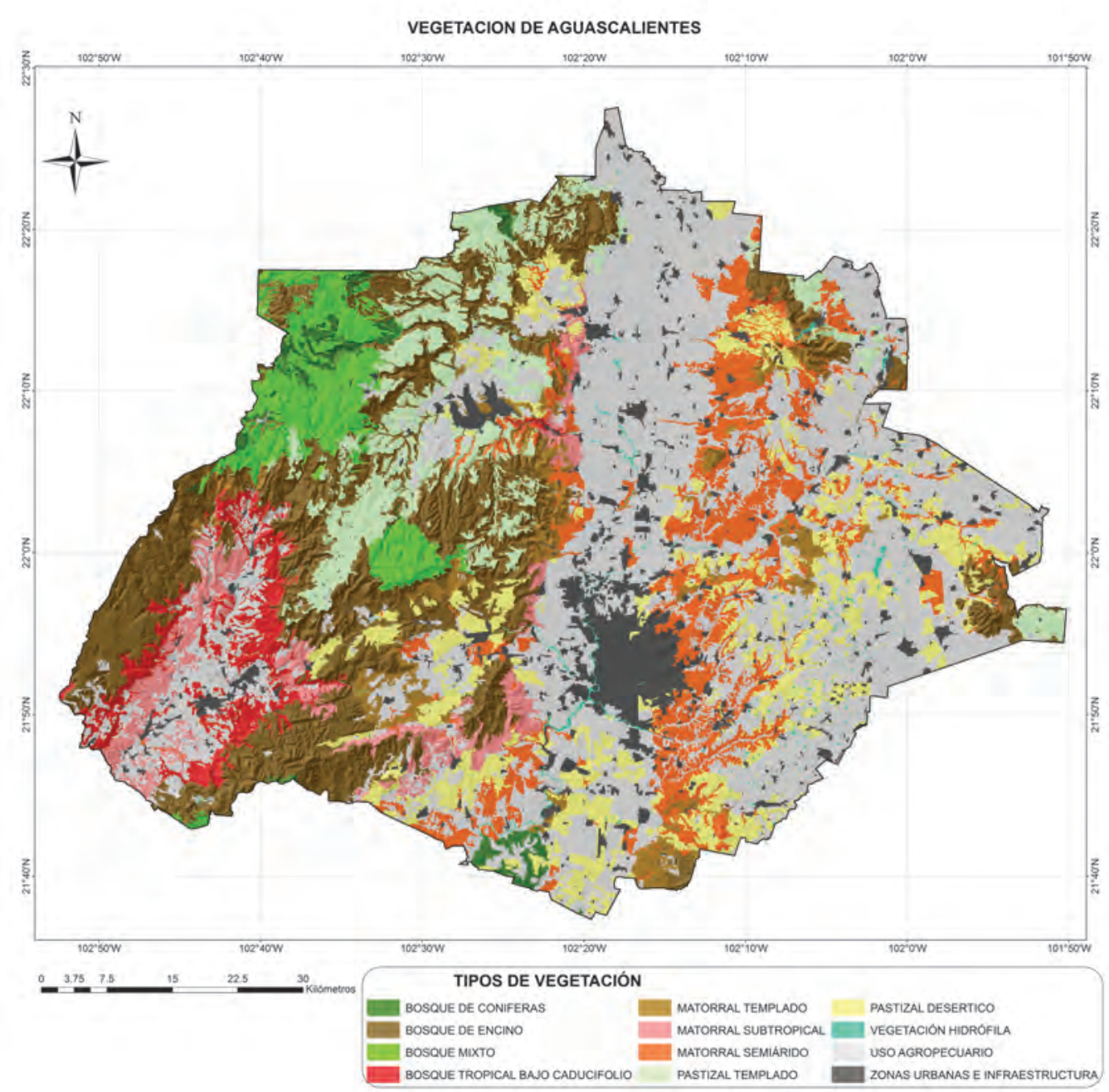

Figura 2. Tipos de vegetación presentes en el estado de Aguascalientes.

(Cupressus lusitanica), o más frecuentemente por juníperos (Juniperus spp.); se localiza en las partes montañosas más elevadas del estado de los 2,500 a los 3,000 m. (3) Bosque mixto: formado por diferentes tipos de asociaciones vegetales (principalmente encinos y coníferas), ocupan casi cualquier parte de la zona serrana y se sitúan en altitudes de los 2,400 a los 2,800 m. (4) Matorral templado: caracterizado por la presencia de chaparrales de manzanita (Arctostaphylos pungens) en las mesetas o laderas pronunciadas de la sierra, a más de 2,500 m de altitud, o matorrales calcícolas de Juniperus coahuilensis en las planicies semiáridas, entre $\operatorname{los} 2,100$ y los 2,400 m. (5) Pastizal templado: localizado en las mesetas y los piedemonte de la zona montañosa del estado, entre los 2,200 y 2,800 m de altitud, muchos de ellos producto de alteraciones antrópicas del bosque para pastoreo. (6) Bosque tropical bajo caducifolio: se localiza en las áreas más conservadas de vegetación subtropical, principalmente en lomeríos, barrancas y laderas escarpadas que se ubican de los 1,600 a los 1,900 m de altitud. (7) Matorral subtropical: prolifera sobre suelos degradados con pendientes ligeras a pronunciadas, generalmente son comunidades secundarias producto de la degradación del bosque tropical, en altitudes que van de los 1,600 a los 2,200 m. (8) Matorral semiárido: se distribuye en la meseta central y porción noroeste del Estado, donde originalmente se establecían pastizales abiertos asociados a mezquitales y nopaleras. Desde el punto de vista fisonómico son comunidades muy variadas y diversas que incluyen matorrales inermes, micrófilos y espinosos crasicaules, en altitudes de los 1,900 a los 2,300 m. (9) Pastizal desértico: está localizado principalmente en el altiplano central de estado, se desarrolla en llanuras y mesetas de toda la región y se considera una de las comunidades más vulnerables por su ubicación y fácil acceso. (10) Vegetación hidrófila: se encuentra asociada a los cuerpos de agua naturales, artificiales, estacionales y permanentes de todo el estado, de todas las comunidades vegetales es la que ocupa menor superficie y la más susceptible al deterioro por las actividades humanas.

Material biológico. Los datos se obtuvieron de 862 ejemplares, de los cuales 235 pertenecen a la colección del herbario 


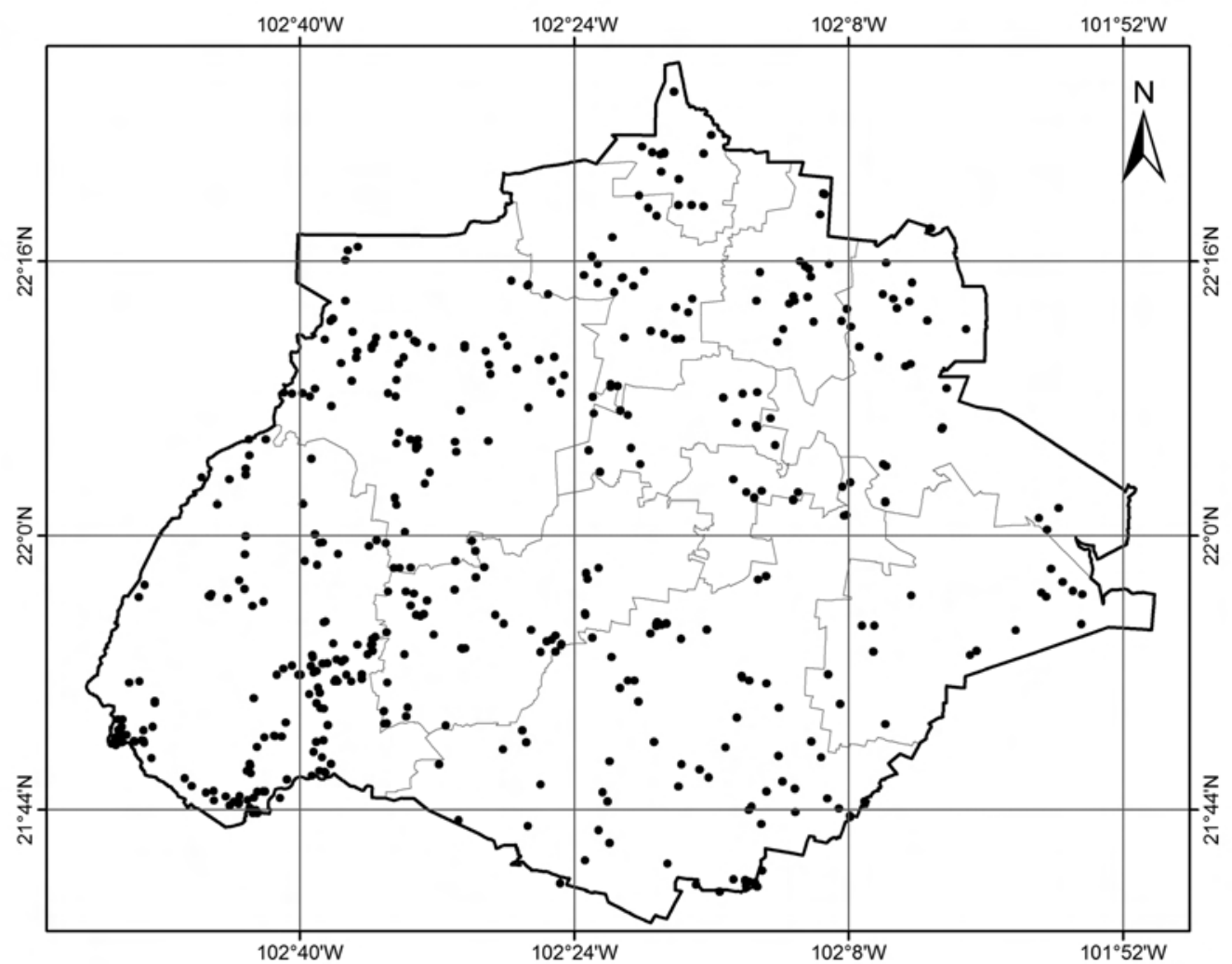

Figura 3. Ubicación de los sitios de colecta de Solanaceae en el estado de Aguascalientes.

HUAA, 38 a las colecciones de los herbarios: ENCB, GUADA, IBUG, IEB, INEGI y MEXU; y 589 números fueron colectados en 312 localidades del estado de Aguascalientes durante el periodo de junio de 2011 a noviembre de 2013 (Figura 3). La determinación de los ejemplares se llevó a cabo principalmente utilizando las claves de identificación de Bates (2009), Correll (1962), Correll y Johnston (1979), Cuevas-Arias et al. (2008), D’Arcy (1973, 1991), Dean (2004), Gentry y Standley (1974), Knapp (2002), Nee (1986, 1993), Rodríguez y Vargas (1994, 2001), Roe (1972), Calderón de Rzedowski y Rzedowski (2005), Shreve y Wiggins (1964), Spooner et al. (2004), Standley (1924), Stevens et al. (2001), Tepe y Bohs (2011), Vargas y Rodríguez (1993), Vargas et al. (2003), Waterfall (1967), Whalen (1979) y Whalen et al. (1981). Los ejemplares colectados fueron depositados en el herbario HUAA y se enviaron ejemplares de intercambio a distintos herbarios de la región.

\section{Resultados}

En Aguascalientes, la familia Solanaceae está representada por 12 géneros (Cuadro 1) y 55 especies, de las cuales 16 resultaron ser nuevos registros para el estado (Cuadro 2), debido a que no se tenía registro de su presencia en el herbario HUAA, en el resto de los herbarios visitados y en bibliografía consultada. Los géneros Solanum y Physalis son los más diversos al poseer el $69 \%$ de las especies. El matorral semiárido fue el tipo de vegetación con mayor riqueza, alberga 32 especies (Figura 4), seguido del matorral subtropical con 30, bosque de encino con 28 y la vegetación hidrófila con 21. Calvillo es el municipio con mayor riqueza, en él se registraron 46 especies de la familia (Figura 5), seguido de Aguascalientes con 29 y San José de Gracia con 22. La familia Solanaceae ocupa el quinto lugar en diversidad de especies en el estado, a pesar de que algunas presentan una distribución restringida.

Cuadro 1. Géneros y especies de Solanaceae en Aguascalientes.

\begin{tabular}{lc}
\hline Género & Número de especies \\
\hline Solanum L. & 23 \\
Physalis L. & 14 \\
Datura L. & 4 \\
Nicotiana L. & 4 \\
Bouchetia Dunal & 2 \\
Lycianthes (Dunal) Hassl. & 2 \\
Cestrum L. & 1 \\
Chamaesaracha (A.Gray) Benth. & 1 \\
Jaltomata Schltdl. & 1 \\
Nicandra Adans. & 1 \\
Nierembergia Ruíz y Pavón & 1 \\
Petunia Juss. & 1 \\
Total & 55
\end{tabular}


Cuadro 2. Especies de Solanaceae en Aguascalientes, distribución y tipos de vegetación en el que habitan. 1: Aguascalientes, 2: Asientos, 3: Calvillo, 4: Cosío, 5: El Llano, 6: Jesús María, 7: Pabellón de Arteaga, 8: Rincón de Romos, 9: San Francisco de Los Romo, 10: San José de Gracia, 11: Tepezalá. BC: bosque de coníferas, BM: bosque mixto, BQ: bosque de encino, BTC: bosque tropical bajo caducifolio, MS: matorral subtropical, MSA: matorral semiárido, MT: matorral templado, PD: pastizal desértico, PT: pastizal templado, VD: vegetación de disturbio, VH: vegetación hidrófila. *Nuevos registros.

\begin{tabular}{|c|c|c|}
\hline Especie & Distribución (municipio) & Tipo de vegetación \\
\hline Bouchetia arniatera B.L.Rob. & $3,6,7,10,11$ & $\mathrm{BM}, \mathrm{BQ}, \mathrm{MSA}, \mathrm{MT}, \mathrm{PD}, \mathrm{PT}$ \\
\hline *Bouchetia erecta Dunal & 1 & PD \\
\hline Cestrum tomentosum L. f. & 3 & BTC, MS, MSA, VH \\
\hline Chamaesaracha coronopus (Dunal) A. ray & $2,4,7,8,9,11$ & MSA, PD, VD \\
\hline Datura ceratocaula Ortega & $1,2,3,5,6,9,10,11$ & MSA, VD, VH \\
\hline Datura inoxia Mill. & $1,3,4,6,8,9,10$ & BTC, MS, MSA, PD,VD, VH \\
\hline Datura quercifolia Kunth & $1,2,3,4,7,8,9$ & $\mathrm{BQ}, \mathrm{BTC}, \mathrm{MS}, \mathrm{MSA}, \mathrm{PD}, \mathrm{PT}, \mathrm{VD}$ \\
\hline Datura stramonium L. & $1,2,3,4,6,10$ & $\mathrm{BM}, \mathrm{BQ}, \mathrm{BTC}, \mathrm{MS}, \mathrm{MSA}, \mathrm{PT}, \mathrm{VD}, \mathrm{VH}$ \\
\hline Jaltomata procumbens (Cav.) J.L.Gentry & $1,3,4,6,10,11$ & $\mathrm{BC}, \mathrm{BM}, \mathrm{BQ}, \mathrm{BTC}, \mathrm{MS}, \mathrm{MSA}, \mathrm{PD}, \mathrm{PT}, \mathrm{VD}, \mathrm{VH}$ \\
\hline Lycianthes dejecta (Fernald) Bitter & $3,5,9$ & $\mathrm{BQ}, \mathrm{MS}, \mathrm{MSA}, \mathrm{PD}, \mathrm{PT}$ \\
\hline Lycianthes moziniana (Dunal) Bitter var. moziniana & 3,10 & $\mathrm{BC}, \mathrm{BM}, \mathrm{BQ}, \mathrm{PT}$ \\
\hline Nicandra physalodes (L.) Gaertn. & 3 & $B Q, V D$ \\
\hline Nicotiana g/auca Graham & $1,2,3,4,5,6,7,8,9,10,11$ & $\mathrm{BQ}, \mathrm{BTC}, \mathrm{MS}, \mathrm{MSA}, \mathrm{PD}, \mathrm{PT}, \mathrm{VD}, \mathrm{VH}$ \\
\hline Nicotiana obtusifolia M.Martens \& Galeotti & $1,2,3,4,5,7,8,9,10,11$ & $\mathrm{BQ}, \mathrm{MS}, \mathrm{MSA}, \mathrm{MT}, \mathrm{PD}, \mathrm{PT}, \mathrm{VD}$ \\
\hline Nicotiana plumbaginifolia Viv. & 1,3 & $\mathrm{VH}, \mathrm{VD}$ \\
\hline Nicotiana tabacum L. & 3 & $\mathrm{BQ}, \mathrm{MS}$ \\
\hline Nierembergia angustifolia Kunth & $1,2,4,6,8,10$ & $\mathrm{VH}$ \\
\hline Petunia parviflora Juss. & $1,2,3,4,6,8,10,11$ & $\mathrm{VH}$ \\
\hline Physalis angulata $\mathrm{L}$. & $1,3,6$ & $\mathrm{BQ}, \mathrm{BTC}, \mathrm{MS}, \mathrm{MSA}, \mathrm{PD}$ \\
\hline Physalis chenopodifolia Lam. & $1,2,3,5,6,10$ & $\mathrm{BM}, \mathrm{BQ}, \mathrm{MS}, \mathrm{MSA}, \mathrm{PD}, \mathrm{PT}$ \\
\hline Physalis cinerascens (Dunal) Hitchc.var. cinerascens & $1,3,7$ & VD \\
\hline Physalis g/utinosa Schltdl. & $2,3,4,5,8,10,11$ & $\mathrm{BM}, \mathrm{BQ}, \mathrm{BTC}, \mathrm{MSA}, \mathrm{PD}, \mathrm{PT}$ \\
\hline Physalis hastatula Waterf. & 5 & MSA, PD \\
\hline Physalis lagascae Roem. \& Schult. & 3 & MS, VH \\
\hline Physalis nicandroides Schltdl. & $1,3,6,8,10$ & $\mathrm{BM}, \mathrm{BQ}, \mathrm{MS}, \mathrm{MSA}, \mathrm{PD}, \mathrm{VD}, \mathrm{VH}$ \\
\hline Physalis orizabae Dunal & $1,2,3,6,9,10$ & $\mathrm{BC}, \mathrm{BM}, \mathrm{BQ}, \mathrm{MSA}, \mathrm{PD}, \mathrm{VD}, \mathrm{VH}$ \\
\hline Physalis patula Mill. & $1,2,3,5,6,7,8,9$ & MS, MSA, PD, VD, VH \\
\hline Physalis philadelphica Lam. & 3,6 & $\mathrm{BQ}, \mathrm{MS}, \mathrm{MSA}, \mathrm{VD}$ \\
\hline *Physalis pruinosa $\mathrm{L}$. & 3 & BTC, MS, MSA \\
\hline *Physalis pubescens $\mathrm{L}$. & 1,3 & $\mathrm{BQ}, \mathrm{MSA}, \mathrm{PD}$ \\
\hline Physalis solanaceus (Schltdl.) Axelius & $1,3,6,7$ & $\mathrm{BQ}, \mathrm{BTC}, \mathrm{MS}, \mathrm{MSA}, \mathrm{PD}, \mathrm{VD}$ \\
\hline *Physalis virginiana Mill. & 7,11 & MSA \\
\hline *Solanum americanum Mill. & $1,3,4,5,6,7,8,10,11$ & $\mathrm{BQ}, \mathrm{BTC}, \mathrm{MSA}, \mathrm{PD}, \mathrm{PT}, \mathrm{VD}, \mathrm{VH}$ \\
\hline Solanum cardiophyllum Lindl. & 1 & PD \\
\hline Solanum corymbosum Jacq. & 11 & MSA, PD \\
\hline * Solanum dasyadenium Bitter & $1,3,4,7,10,11$ & $\mathrm{BM}, \mathrm{BQ}, \mathrm{MS}, \mathrm{MSA}, \mathrm{VH}$ \\
\hline *Solanum deflexum Greenm. & 3 & BTC, MS \\
\hline Solanum demissum Lindl. & 10 & $\mathrm{BQ}$ \\
\hline *Solanum dulcamaroides Dunal & 3 & MS \\
\hline Solanum ehrenbergii (Bitter) Rydb. & $1,3,11$ & $\mathrm{BQ}, \mathrm{BTC}, \mathrm{PD}, \mathrm{VD}, \mathrm{VH}$ \\
\hline Solanum elaeagnifolium Cav. & $1,2,3,4,5,6,7,8,9,10,11$ & $\mathrm{BQ}, \mathrm{MS}, \mathrm{MSA}, \mathrm{PD}, \mathrm{PT}, \mathrm{VD}, \mathrm{VH}$ \\
\hline *Solanum erianthum D.Don & 3 & MS \\
\hline *Solanum ferrugineum Jacq. & 3 & BTC, MS \\
\hline *Solanum grayi Rose var. grandiflorum Whalen & 3 & $\mathrm{BQ}, \mathrm{MS}$ \\
\hline *Solanum heterodoxum Dunal & $1,2,4$ & MSA \\
\hline *Solanum houstonii Martyn & 3 & BQ \\
\hline *Solanum lycopersicum L. & 3 & $\mathrm{VD}, \mathrm{VH}$ \\
\hline Solanum nigrescens M.Martens \& Galeotti & $1,3,5,6,8,10$ & $\mathrm{BC}, \mathrm{BM}, \mathrm{BQ}, \mathrm{MS}, \mathrm{MSA}, \mathrm{VD}, \mathrm{VH}$ \\
\hline *Solanum polyadenium Greenm. & 3 & MS \\
\hline *Solanum pseudocapsicum L. & 3 & $B Q, M S$ \\
\hline Solanum rostratum Dunal & $1,2,3,4,5,6,7,8,9,10,11$ & $\mathrm{BM}, \mathrm{BQ}, \mathrm{BTC}, \mathrm{MS}, \mathrm{MSA}, \mathrm{PD}, \mathrm{PT}, \mathrm{VD}, \mathrm{VH}$ \\
\hline Solanum stenophyllidium Bitter & $1,3,4,6,7,8,10$ & $\mathrm{BQ}, \mathrm{BTC}, \mathrm{MS}, \mathrm{MSA}, \mathrm{PD}, \mathrm{PT}, \mathrm{VD}, \mathrm{VH}$ \\
\hline Solanum stoloniferum Schltdl. & $1,3,5,10,11$ & $\mathrm{BC}, \mathrm{BM}, \mathrm{BQ}, \mathrm{MSA}, \mathrm{PT}$ \\
\hline Solanum trifidum Correll & 3 & $\mathrm{BQ}$ \\
\hline Solanum umbellatum Mill. & 3 & $\mathrm{BQ}, \mathrm{BTC}, \mathrm{MS}$ \\
\hline
\end{tabular}




\section{Discusión}

La familia Solanaceae se encuentra ampliamente distribuida en el estado de Aguascalientes, se le puede encontrar desde los 1,600 m, en el municipio de Calvillo, hasta los 3,000 metros de altitud, en el municipio de San José de Gracia. Está presente en todas las comunidades vegetales que se desarrollan en el Estado, tanto en áreas conservadas como con diferentes grados de disturbio.

Muchas de las especies de la familia se desarrollan como malezas ruderales y arvenses, de tal manera, que es muy frecuente encontrarlas en las zonas con disturbio en todos los tipos de vegetación presentes en el estado, así como en zonas urbanas. Entre las más comunes están Datura inoxia, D. quercifolia, D. stramonium, Nicotiana glauca, N. obtusifolia, Physalis angulata, P. chenopodifolia, P. cinerascens var. cinerascens, $P$. nicandroides, $P$. patula, $P$. philadelphica, $P$. solanaceus, Solanum americanum, S. elaeagnifolium, $S$. nigrescens y $S$. rostratum. Otras especies menos frecuentes, no siempre consideradas como malezas, pero que también se pueden encontrar en zonas degradadas son: Lyciantes dejecta, L. moziniana var. moziniana, Nicandra physalodes, Nicotiana plumbaginifolia, N. tabacum, Physalis hastatula, P. lagascae, $P$. orizabae, $P$. pruinosa, $P$. pubescens, $P$. virginiana, Solanum cardiophyllum, S. ehrenbergii, S. erianthum, S. grayi var. grandiflorum, S. heterodoxum, S. houstonii, S. lycopersicum, S. nigrescens, S. stenophyllidium, S. stoloniferum y $S$. umbellatum.

Es posible encontrar también representantes de la familia en áreas relativamente conservadas de bosque de encino, entre las que se pueden mencionar Bouchetia arniatera, que prefiere áreas abiertas, Lycianthes moziniana var. moziniana en áreas con cierto grado de disturbio, Physalis glutinosa en áreas con pendientes rocosas, así como Solanum stenophyllidium y S. stoloniferum, que son las más comunes y más ampliamente distribuidas. Las especies $S$. demissum y $S$. trifidum fueron colectadas únicamente en el bosque de encino, en las áreas mejor conservadas y de mayor altitud (2,989 y $2,500 \mathrm{~m}$, respectivamente). Ciertas especies prefieren zonas de ecotonía de bosque de encino con bosque tropical bajo caducifolio y con matorral subtropical, como son L. dejecta, S. grayi var. grandiflorum, $S$. houstonii, S. pseudocapsicum y $S$. umbellatum. En las zonas de bosque mixto, se pueden localizar especies como Jaltomata procumbens en las cercanías de arroyos; $P$. orizabae, $S$. dasyadenium y $S$. nigrescens en zonas húmedas donde se acumula la materia orgánica. El bosque de encino representa uno de los tipos de vegetación más importantes en el estado y más de la mitad de su superficie se encuentra con un alto grado de perturbación (Rodríguez-Ávalos, 2014).

Por otro lado, hay ciertas especies que prefieren ambientes subtropicales. En el estado de Aguascalientes, estas áreas están representadas por el bosque tropical bajo caducifolio y el matorral subtropical. Ambos tipos de vegetación se encuentran estrechamente relacionadas a condiciones de mayor temperatura, donde se pueden encontrar especies como Cestrum tomentosum, que puede alcanzar hasta más de $10 \mathrm{~m}$ de altura; Jaltomata procumbens, que crece entre las rocas, Solanum ferrugineum en zonas sombreadas donde puede llegar a formar poblaciones amplias y $S$. umbellatum que prefiere sustratos arenosos. El matorral subtropical presenta mayor riqueza de especies de Solanaceae que el bosque tropical bajo caducifolio, debido a que el matorral se desarrolla en respuesta a la degradación del bosque, lo que genera el ambiente propicio

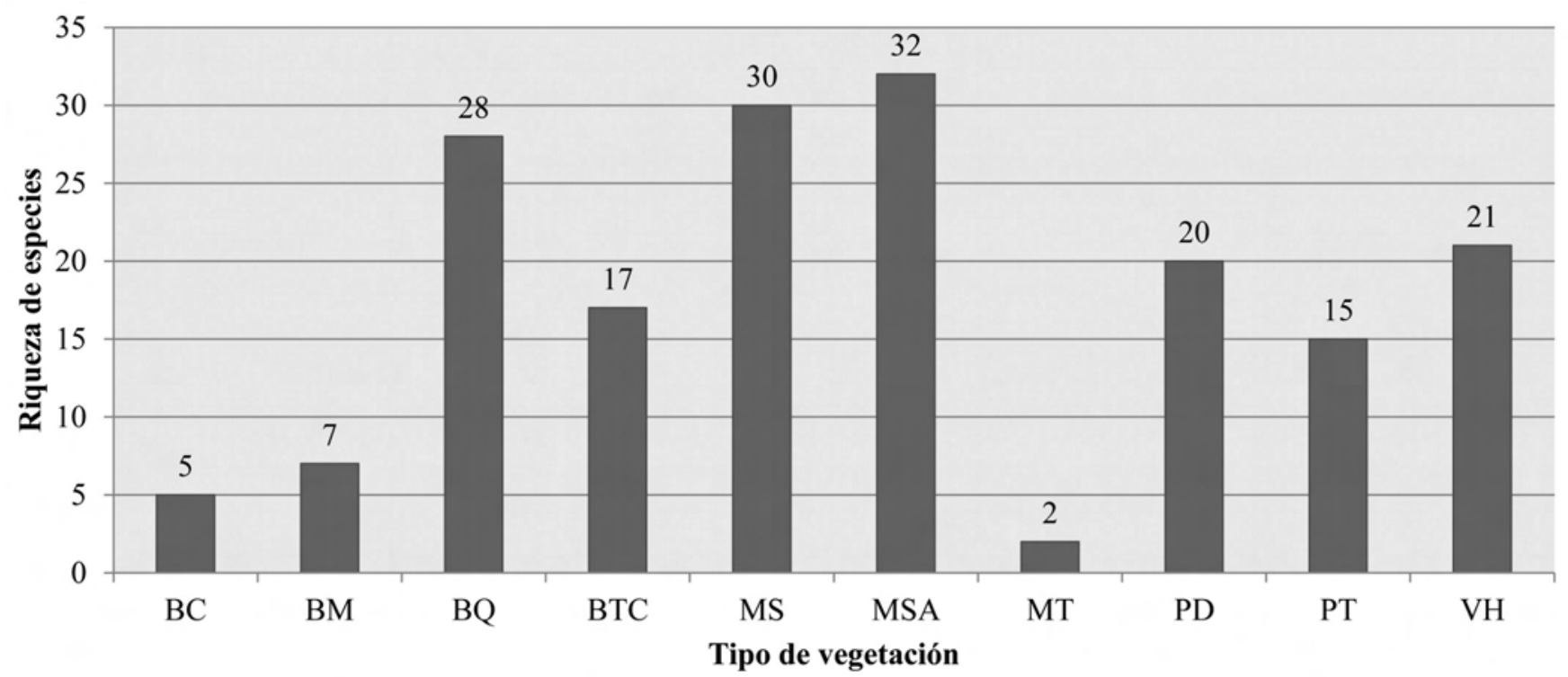

Figura 4. Riqueza de especies de Solanaceae por tipo de vegetación del estado de Aguascalientes. BC: bosque de coníferas, BM: bosque mixto, BQ: bosque de encino, BTC: bosque tropical bajo caducifolio, MS: matorral subtropical, MSA: matorral semiárido, MT: matorral templado, PD: pastizal desértico, PT: pastizal templado, VH: vegetación hidrófila. 


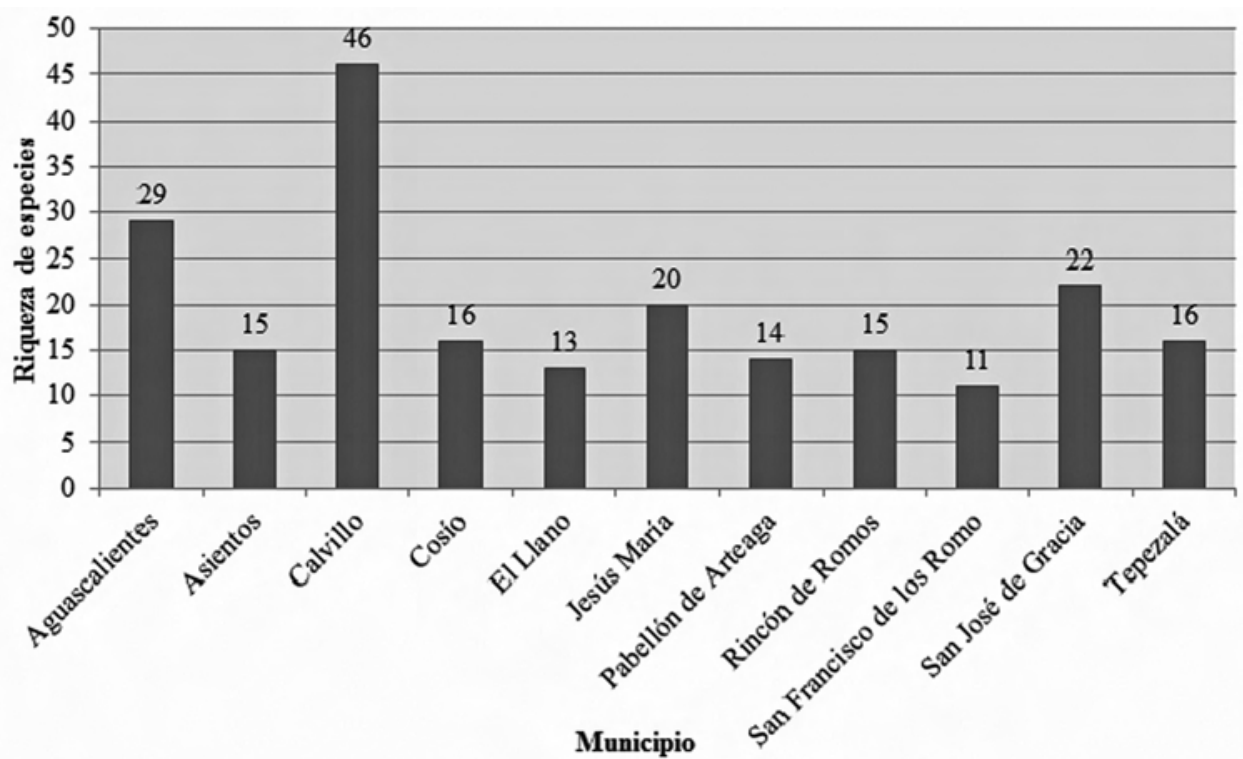

Figura 5. Riqueza de especies de Solanaceae por municipios del estado de Aguascalientes.

para el establecimiento de otras especies poco frecuentes en estas áreas, como Lycianthes dejecta, Physalis lagascae, $P$. pruinosa, S. dasyadenium, S. dulcamaroides, S. erianthum, $S$. grayi var. grandiflorum, $S$. nigrescens, $S$. polyadenium, $S$. pseudocapsicum y S. stenophyllidium.

La familia Solanaceae encuentra su mayor representación en la vegetación xerófila del estado, particularmente en los matorrales semiáridos y pastizales desérticos. Aunque en Aguascalientes se presentan diferentes tipos de matorral, la mayoría de ellos son generalmente producto de la degradación de la vegetación original; o bien, sus comunidades originales se encuentran en un grado de perturbación considerable (Rodríguez-Ávalos, 2014). Las zonas más accesibles, y que son utilizadas principalmente para el pastoreo extensivo, son las que muestran los mayores grados de perturbación y con las poblaciones de Solanaceae más amplias. En estas áreas es posible encontrar especies como Chamaesaracha coronopus en zonas secas con sustratos rocosos, Jaltomata procumbens en las áreas húmedas cercanas a corrientes de agua estacionales, Physalis hastatula y Solanum stenophyllidium en zonas con un estrato arbustivo más cerrado y protegidas por la vegetación; de manera particular, S. corymbosum únicamente se encontró en zonas ruderales del matorral desértico micrófilo, que se ubica en la parte noreste del estado y que es continuación del desierto chihuahuense.

Algunas especies como Datura ceratocaula, Nierembergia angustifolia y Petunia parviflora, se localizan únicamente en zonas con humedad en el sustrato, como son orillas de arroyos y bordos temporales o permanentes, márgenes de presas, así como áreas inundables. Otras especies que también están relacionadas a los cuerpos de agua son Cestrum tomentosum, Jaltomata procumbens, Nicotiana plumbaginifolia, Solanum americanum, S. ferrugineum, $S$. pseudocapsicum y S. umbellatum.
La gran mayoría de las especies de la familia Solanaceae no tiene problemas de supervivencia en el estado y presentan una distribución muy amplia, de tal manera que se registraron en todos o casi todos los municipios. Sin embargo, existen casos particulares de especies con distribución más restringida en el estado, como son Cestrum tomentosum, Nicandra physalodes, Nicotiana tabacum, Physalis lagascae, P. pruinosa, Solanum deflexum, S. dulcamaroides, S. erianthum, S. ferrugineum, S. grayi var. grandiflorum, S. houstonii, S. lycopersicum, S. polyadenium, S. pseudocapsicum, S. trifidum y S. umbellatum, que se colectaron únicamente en el municipio de Calvillo; Bouchetia erecta y $S$. cardiophyllum en Aguascalientes; $P$. hastula en El Llano; S. demissum en San José de Gracia; y $S$. corymbosum en Tepezalá. A $S$. dulcamaroides se le asignó el estatus de "rara" en el Estado, debido a que está representada por un único ejemplar depositado en el herbario HUAA y colectado en matorral subtropical en 1981; a pesar de que se realizaron muestreos en la misma localidad, áreas circundantes y diversas localidades con este tipo de vegetación, no se ubicó nuevamente, debido probablemente al grado de perturbación actual del área donde se colectó por primera vez.

Algunas especies de la familia Solanaceae se aprovechan de manera local con diversos fines, tal es el caso de los frutos de Jaltomata procumbens, Physalis philadelphica, Solanum lycopersicum, y los tubérculos de S. cardiophyllum, S. ehrenbergii y $S$. stenophyllidium, que son utilizados con fines alimenticios. Por otro lado, diversas partes de Datura quercifolia, D. inoxia, D. stramonium, Lycianthes dejecta, $L$. moziniana var. moziniana, $P$. angulata, $P$. cinerascens var. cinerascens, $S$. elaeagnifolium, $S$. nigrescens y $S$. rostratum, son usadas con fines medicinales (Barba et al., 2003; García, 2014). Asimismo, especies como D. inoxia y D. stramonium 
se toleran en jardines particulares con fines ornamentales, debido a su abundante follaje y llamativa floración.

\section{Agradecimientos}

Se agradece al CONACYT por la beca otorgada (CVU 444488), al programa "Maestría en Ciencias Agronómicas y Veterinarias" de la Universidad Autónoma de Aguascalientes, a Gerardo García Regalado por las sugerencias hechas al manuscrito, a Alberto Rodríguez Ávalos por el apoyo en la elaboración de los mapas y al personal del herbario HUAA. Asimismo, se agradece a los revisores del manuscrito por sus atinadas recomendaciones para el mejoramiento del mismo.

\section{Literatura citada}

Barba Á.M.D., Croce H.D.M. y de la Cerda L.M. 2003. Plantas Útiles de la Región Semiárida de Aguascalientes. Universidad Autónoma de Aguascalientes, Aguascalientes.

Bates S.T., Farruggia F, Gilbert E., Gutierrez R., Jenke D., Makings E., Manton E., Newton D. y Landrum L.R. 2009. Solanaceae potato family. Part two: Key to the genera and Solanum L. Canotia 5:1-16.

Calderón de Rzedowski G. y Rzedowski J. 2005. Flora Fanerogámica del Valle de México. 2a . ed. Instituto de Ecología, A.C., Comisión Nacional para el Conocimiento y Uso de la Biodiversidad, Pátzcuaro.

CONABIO. Comisión Nacional para el Conocimiento y Uso de la Biodiversidad. 2008. La Biodiversidad en Aguascalientes: Estudio de estado. Comisión Nacional para el Conocimiento y Uso de la Biodiversidad, Instituto del Medio Ambiente del estado de Aguascalientes, Universidad Autónoma de Aguascalientes. México, D.F.

Correll D.S. 1962. The potato and its wild relatives. Botanical Studies Vol. 4. Contributions of the Texas Research Foundation, Dallas.

Correll D.S. y Johnston M.C. 1979. Manual of the Vascular Plants of Texas. The University of Texas, Austin.

Cuevas-Arias C.T., Vargas O. y Rodríguez A. 2008. Solanaceae diversity in the state of Jalisco, Mexico. Revista Mexicana de Biodiversidad 79:67-79.

D'Arcy W.G. 1973. Solanaceae. Flora of Panama. Part IX. Annals of the Missouri Botanical Garden 60:573-780.

D'Arcy W.G. 1991. The Solanaceae since 1976, with a review of its biogeography. En: Hawkes J.G., Lester R.N., Nee M. y EstradaR. N. Eds. Solanaceae III: Taxonomy, Chemistry and Evolution, pp. 75-137, The Royal Botanical Gardens, Kew, Londres.

Dean E.A. 2004. A taxonomic revision of Lycianthes series Meizonodontae (Solanaceae). Botanical Journal of the Linnean Society 145:385-424.

de la Cerda L.M. 1982. Estudio Taxonómico Ecológico de la Flora y Fauna del Estado de Aguascalientes. Universidad Autónoma de Aguascalientes, Aguascalientes.

de la Cerda L.M. 1996. Las Gramíneas de Aguascalientes. Universidad Autónoma de Aguascalientes, Aguascalientes.

de la Cerda L.M. 1999a. Cactáceas de Aguascalientes. $2^{\mathrm{a}}$ ed. Universidad Autónoma de Aguascalientes, Aguascalientes. de la Cerda L.M. 1999b. Encinos de Aguascalientes. $2^{a}$ ed. Universidad Autónoma de Aguascalientes, Aguascalientes.

de la Cerda L.M. 2004. Contribución al conocimiento de la flora del estado de Aguascalientes: Familias Agavaceae, Alliaceae, Amaryllidaceae, Anthericaceae, Asphodelaceae, Calochortaceae, Hyacinthaceae, Hypoxidaceae, Melanthiaceae y Nolinaceae. Scientiae Naturae 6:19-106.

de la Cerda M. 2011. Familia Euphorbiaceae en el Estado de Aguascalientes, México. Universidad Autónoma de Aguascalientes, Aguascalientes.

Eckart E. 2008. Solanaceae and Convolvulaceae: Secundary metabolites. Springer-Verlag, Berlín.

Encina-Domínguez J.A., Zarate-Lupercio A., Valdéz-Reyna J. y Villareal-Quintanilla J.A. 2007. Caracterización ecológica y diversidad de los bosques de encino de la sierra de Zapalinamé. Coahuila, México. Boletín de la Sociedad Botánica de México 81:51-63.

Franco O.V.H., Siqueiros D.M.E. y Hernández A.E.G. 2012. Flora Apícola del Estado de Aguascalientes. Universidad Autónoma de Aguascalientes, Aguascalientes.

García R.G. 1998. La familia Loranthaceae (injertos) del estado de Aguascalientes, México. Polibotánica 7:1-14.

García R.G. 2004. Contribución al conocimiento de la flora del estado de Aguascalientes: Familias Clethraceae, Geraniaceae, Rafflesiaceae y Saururaceae. Scientiae Naturae 6:107-130.

García R.G. 2005. Asteraceae: Las Compuestas de Aguascalientes. Universidad Autónoma de Aguascalientes, Aguascalientes.

García R.G. 2014. Las Plantas Medicinales de Aguascalientes. Universidad Autónoma de Aguascalientes, Aguascalientes.

Gentry J.L.Jr. y Standley P.C. 1974. Solanaceae. Flora of Guatemala Part X. Fieldiana: Botany 24:1-151.

González-Adame G. 2004. Contribución al conocimiento de la flora del estado de Aguascalientes: Familias Loasaceae, Loganiaceae y Caprifoliaceae. Scientiae Naturae 6:131-153.

Knapp S. 2002. Solanum section Geminata (Solanaceae). Flora Neotropica 84:1-404.

Long T.J. 2001. Una semblanza de las Solanaceae. Etnobiología 1:18-24.

Nee M. 1986. Solanaceae I. Flora de Veracruz 49:1-191.

Nee M. 1993. Solanaceae II. Flora de Veracruz 72:1-158.

Rodríguez C.A. y Vargas P.O. 1994. Las especies de papa silvestre (Solanum sección Petota Dumortier) en Jalisco. Boletín del Instituto de Botánica 2:1-68.

Rodríguez C.A. y Vargas P.O. 2001. Nuevos registros de Solanum L. (Solanaceae) para el Bajío y regiones adyacentes. Acta Botanica Mexicana 56:1-8.

Rodríguez-Ávalos J.A. 2014. Análisis espacial de la vegetación de Aguascalientes: Distribución geográfica y descripción de las comunidades vegetales naturales de Aguascalientes. Tesis Doctoral, Centro de Ciencias Básicas, Universidad Autónoma de Aguascalientes, Aguascalientes. 295 pp.

Roe K.E. 1972. A revision of Solanum section Brevantherum (Solanaceae). Brittonia 24:239-278.

Shreve F. y Wiggins I.L. 1964. Vegetation and Flora of the Sonoran Desert. Vol. 2. Stanford University Press, Palo Alto.

Siqueiros D.M.E. 1996. Leguminosas de Aguascalientes. Universidad Autónoma de Aguascalientes, Aguascalientes.

Siqueiros D.M.E. 1999. Coníferas de Aguascalientes. $2^{\text {a }}$ ed. Universidad Autónoma de Aguascalientes, Aguascalientes.

Siqueiros D.M.E. y González A.G. 2006. Helechos y Plantas afi- 
nes de Aguascalientes. Universidad Autónoma de Aguascalientes, Aguascalientes.

Siqueiros D.M.E., García R.G., Macías F.C. y Rosales C.O. 2011. Malvales del Estado de Aguascalientes: Bombacaceae, Cistaceae, Malvaceae, Sterculiaceae y Tiliaceae. Universidad Autónoma de Aguascalientes, Aguascalientes.

Spooner D.M., van den Berg R.G., Rodríguez A., Bamberg J., Hijmans R.J. y Lara C.S.I. 2004. Wild potatoes (Solanum section Petota; Solanaceae) of North and Central America. Systematic Botany Monographs 68:1-209.

Standley P.C. 1924. Trees and Shrubs of Mexico (PassifloraceaeScrophulariaceae). Contributions from the United States National Herbarium 23:849-1312.

Stevens W.D., Ulloa C., Pool A. y Montiel O.M. 2001. Flora de Nicaragua. Monographs in Systematic Botany from the Missouri Botanical Garden 85:1-2666.

Tepe E.J. y Bohs L. 2011. A revision of Solanum section Herpysti- chum. Systematic Botany 36:1068-1087.

Toledo V.M. y Ordóñez M.J. 1993. The biodiversity scenario of Mexico: a review of terrestrial habitats. En: Ramamoorthy T.P., Bye R., Lot A. y Fa J. Eds. Biological Diversity of Mexico: Origins and Distribution, pp. 757-777, Oxford University Press, Nueva York.

Vargas O. y Rodríguez A. 1993. La sección Solanum del género Solanum en Jalisco. Boletín del Instituto de Botánica 1:423440.

Vargas P.O., Martínez M. y Dávila A.P. 2003. La familia Solanaceae en Jalisco. El género Physalis. Flora de Jalisco 16:1-127.

Waterfall U.T. 1967. Physalis in Mexico, Central America and the West Indies. Rhodora 69:319-329.

Whalen M.D. 1979. Taxonomy of Solanum section Androceras. Gentes Herbarum 11:359-426.

Whalen M.D., Costich D.E, y Heiser C.B. 1981. Taxonomy of Solanum section Lasiocarpa. Gentes Herbarum 12:41-129.

Recibido: 12 de febrero de 2014

Aceptado: 7 de junio de 2014 
José CARlos Sierra-Muñoz et AL.

Apéndice I. Ejemplares examinados.

Bouchetia arniatera. Mpio. Calvillo: 6 km al S de la presa El Capulín, 2149'01”N, 102³4'55”W, G. García 2731 (HUAA); 1 km

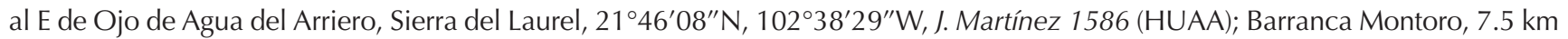
al NW de Milpillas, 21 $59^{\prime} 44^{\prime \prime} \mathrm{N}, 102^{\circ} 35^{\prime} 31^{\prime \prime} \mathrm{W}$, Sierra-Muñoz 644 (HUAA); La Ciénega, $8.5 \mathrm{~km}$ al NE de El terrero del Refugio,

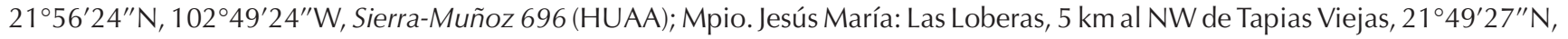

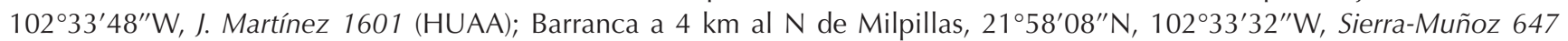
(HUAA); Mpio. Pabellón de Arteaga: $1 \mathrm{~km}$ al N de Santa Cruz, $21^{\circ} 07^{\prime} 32^{\prime \prime} \mathrm{N}, 102^{\circ} 15^{\prime} 43^{\prime \prime} \mathrm{W}, \mathrm{MES} 2876$ (HUAA); Mpio. San José

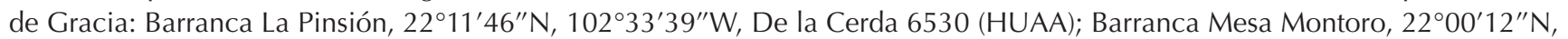

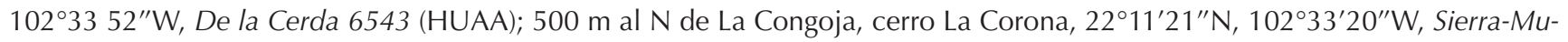

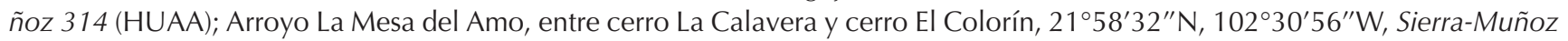
340 (HUAA); 3 km al SO de la Estación Biológica "Agua Zarca", 2205'20"N, 102³4'22"W, Sierra-Muñoz 363 (HUAA); Rancho Sierra Hermosa, 6 km al NW de La Congoja, 22 ${ }^{\circ} 10^{\prime} 44^{\prime \prime} \mathrm{N}, 102^{\circ} 36^{\prime} 40^{\prime \prime} \mathrm{W}$, Sierra-Muñoz 693 (HUAA); Mpio. Tepezalá: El Ranchito, sur de Tepezalá, $22^{\circ} 12^{\prime} 28^{\prime \prime} \mathrm{N}, 102^{\circ} 10^{\prime} 02^{\prime \prime} \mathrm{W}$, De la Cerda 7126 (HUAA). Bouchetia erecta. Mpio. Aguascalientes: Ladera NE del cerro Los Gallos, Sierra-Muñoz 808 (HUAA). Cestrum tomentosum. Mpio. Calvillo: El tepozán, 2156.472” N, 10245.316”W,

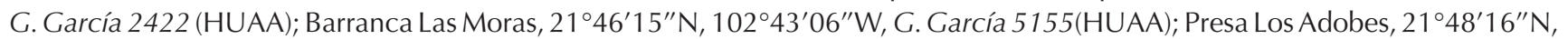

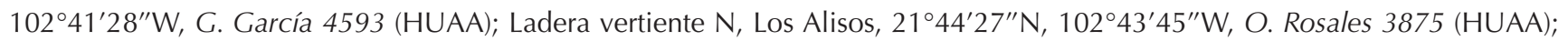
Orilla de arroyo Malpaso, 21 $51^{\prime} 52^{\prime \prime} \mathrm{N}, 102^{\circ} 41^{\prime} 20^{\prime \prime} \mathrm{W}$, De la Cerda 6019 (HUAA); Los Alisos, ladera exposición Norte,

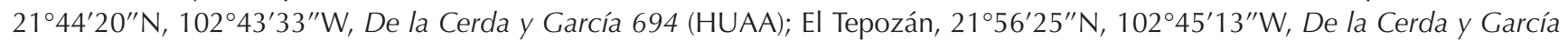

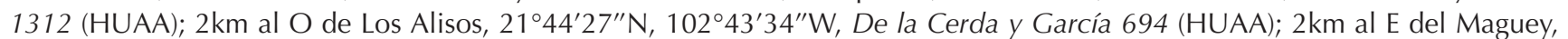

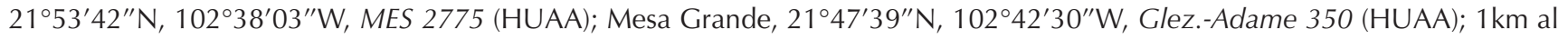

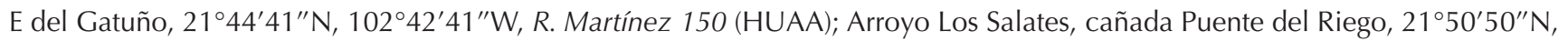
$102^{\circ} 38^{\prime} 50^{\prime \prime} \mathrm{W}$, Sierra-Muñoz 565 (HUAA); Parte media de la barranca La Botita, 4km al SE de Malpaso, 21⒋ $49^{\prime} 54^{\prime \prime} \mathrm{N}, 102^{\circ} 38^{\prime} 36^{\prime \prime} \mathrm{W}$, Sierra-Muñoz 593 (HUAA); Arroyo Malpaso, J. Martínez 1363 (INEGI); El Tepozán, De la Cerda-García 1312 (IEB). Chamaesara-

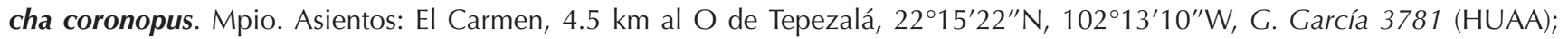
Mpio. Cosío: Ladera media, al E de Cerro Mesa Verde, $22^{\circ} 20^{\prime} 48^{\prime \prime} \mathrm{N}, 102^{\circ} 17^{\prime} 54^{\prime \prime} \mathrm{W}$, De la Cerda 7641 (HUAA); Ladera exposición E del cerro Mesillas, cerca de El Salitrillo, I. García 2156 (INEGI); Mpio. Pabellón de Arteaga: Faldas del cerro del Chiquihuite, $22^{\circ} 06^{\prime} 15^{\prime \prime} \mathrm{N}, 102^{\circ} 13^{\prime} 21^{\prime \prime} \mathrm{W}$, Rosales \& Croce 207 (HUAA); Extremo Norte del rancho Ojo Zarco, carr. Ags.-Asientos, 22 ${ }^{\circ} 06^{\prime} 34^{\prime \prime} \mathrm{N}$,

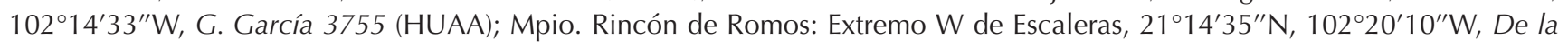
Cerda 5482 (HUAA); Extremo NW de la Estancia de Mosqueira, 22 $08^{\prime} 42^{\prime \prime} \mathrm{N}, 102^{\circ} 21^{\prime} 28^{\prime \prime} \mathrm{W}$, Sierra-Muñoz 423 (HUAA). Datura ceratocaula. Mpio. Aguascalientes: Buena Vista de Peñuelas, $21^{\circ} 45^{\prime} 52^{\prime \prime} \mathrm{N}, 102^{\circ} 16^{\prime} 09^{\prime \prime} \mathrm{W}$, F. Rodríguez 21 (HUAA); Mpio. Calvi-

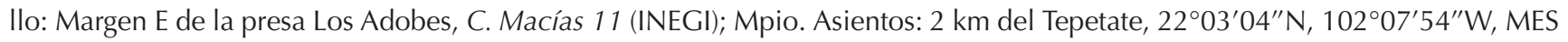

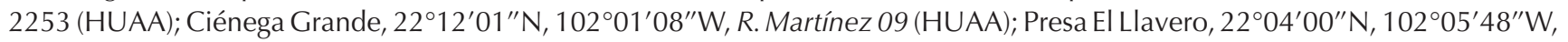
Sierra-Muñoz 436 (HUAA); Mpio. Jesús María: Extremo NW de Milpillas de Abajo, 2156.43’ N, 102³3.50’ W, G. García 5493 (HUAA); Mpio. San José de Gracia: 11 km al NE de San Antonio de Los Ríos, 22¹0.24’ N, 102²6.05’, G. García 5315 (HUAA); Mpio. Tepezalá: NW de San Rafael de Ocampo, 22 $2^{\circ} 3^{\prime} 54^{\prime \prime} \mathrm{N}, 102^{\circ} 10^{\prime} 23^{\prime \prime}$ W, MES 4242 (HUAA). Datura inoxia. Mpio. Aguasca-

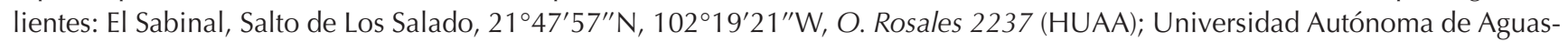

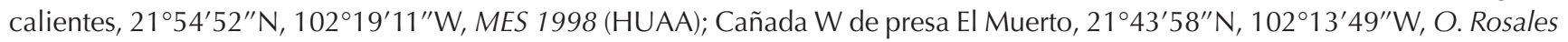

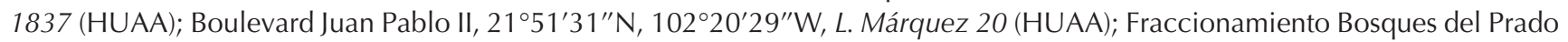

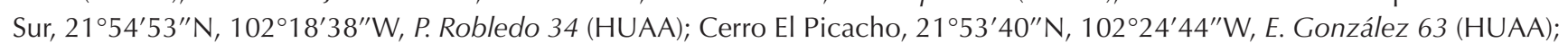

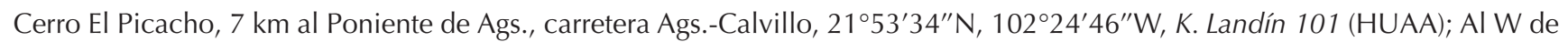

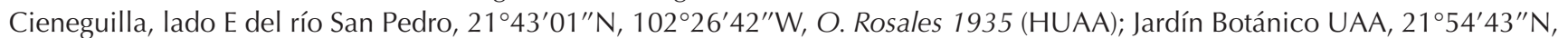

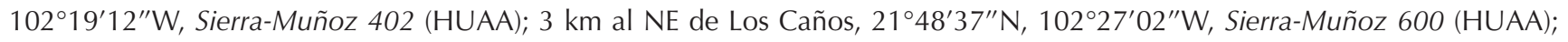

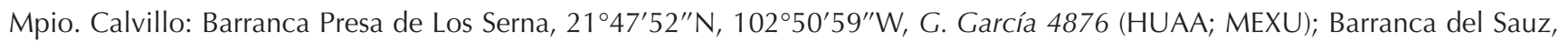

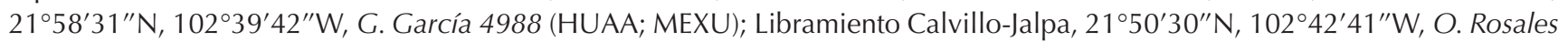

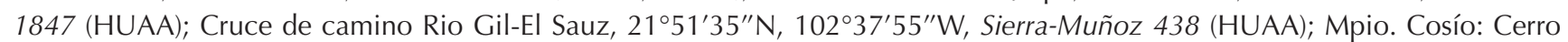
Mesa Verde al W del poblado La Punta, $22^{\circ} 19^{\prime} 13^{\prime \prime} \mathrm{N}, 102^{\circ} 16^{\prime} 28^{\prime \prime} \mathrm{W}$, O. Rosales 2036 (HUAA); Tanque La Punta, lado E del poblado La Punta, $22^{\circ} 19^{\prime} 16^{\prime \prime} \mathrm{N}, 102^{\circ} 17^{\prime} 09^{\prime \prime} \mathrm{W}$, O. Rosales 2060 (HUAA); Mpio. Jesús María: Cerro La Presa, presa el Garabato, $22^{\circ} 04^{\prime} 768^{\prime \prime} \mathrm{N}, 102^{\circ} 20^{\prime} 848^{\prime \prime} \mathrm{W}$, De la Cerda 6237 (HUAA); Presa Abelardo Rodríguez, 21 ${ }^{\circ} 54^{\prime} 29^{\prime \prime} \mathrm{N}, 102^{\circ} 26^{\prime} 31^{\prime \prime} \mathrm{W}$, A. Weeke 82

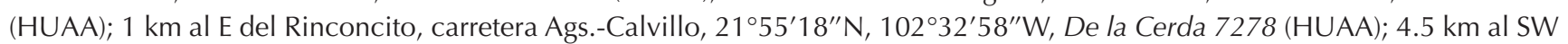
de Jesús María, $21^{\circ} 57^{\prime} 47^{\prime \prime} \mathrm{N}, 102^{\circ} 23^{\prime} 18^{\prime \prime}$ W, Sierra-Muñoz 548 (HUAA). Datura quercifolia. Mpio. Aguascalientes: Residencial

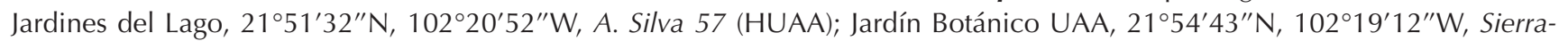
Muñoz 388 (HUAA); Mpio. Asientos: Bordo al Extremo SW de Plutarco Elías Calles, 22 ${ }^{\circ} 12^{\prime} 32^{\prime \prime} \mathrm{N}, 102^{\circ} 03^{\prime} 25^{\prime \prime} \mathrm{W}, 1.2^{\prime}$ Ortiz 01 
Solanaceae en Aguascalientes, México

Apéndice I. Continuación.

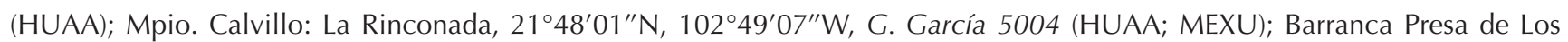

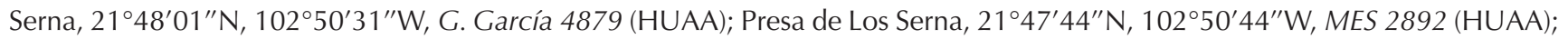

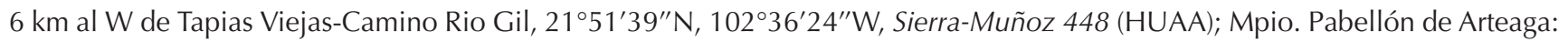

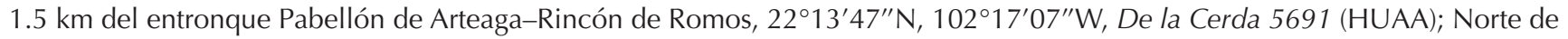
Ojo Zarco carretera a Asientos, 22 $2^{\circ} 8^{\prime} 16^{\prime \prime} \mathrm{N}, 102^{\circ} 14^{\prime} 10^{\prime \prime} \mathrm{W}$, De la Cerda 5491 (HUAA); Mpio. Rincón de Romos: $3 \mathrm{~km}$ al W de Boquillas, $22^{\circ} 16^{\prime} 18^{\prime \prime} \mathrm{N}, 102^{\circ} 22^{\prime} 58^{\prime \prime} \mathrm{W}$, De la Cerda 6321 (HUAA). Datura stramonium. Mpio. Aguascalientes: Jardín Botánico UAA, $21^{\circ} 54^{\prime} 43^{\prime \prime} \mathrm{N}, 102^{\circ} 19^{\prime} 12^{\prime \prime}$ W, Sierra-Muñoz 387 (HUAA); Nuevo jardín botánico UAA, G. García 5301 (IEB); Mpio. Calvillo:

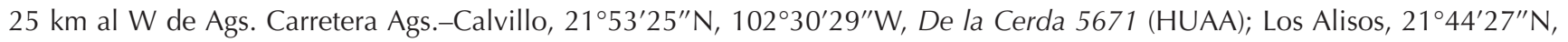

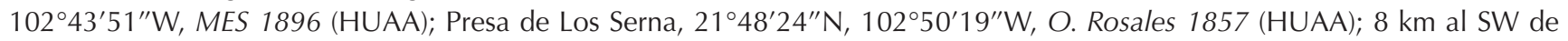
Tapias Viejas, $21^{\circ} 51^{\prime} 52^{\prime \prime} \mathrm{N}, 102^{\circ} 37^{\prime} 16^{\prime \prime} \mathrm{W}$, Sierra-Muñoz 462 (HUAA); 2 km al E del Sauz, barranca Cebolletas, 21 ${ }^{\circ} 53^{\prime} 39^{\prime \prime} \mathrm{N}$,

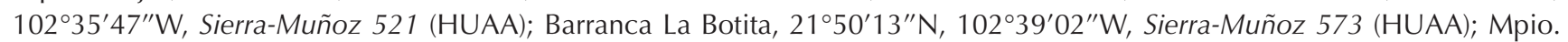
Cosío: $2 \mathrm{~km}$ al SW de Cosío, $22^{\circ} 21^{\prime} 13^{\prime \prime} \mathrm{N}, 102^{\circ} 48^{\prime} 56^{\prime \prime} \mathrm{W}, \mathrm{H}$. Araiza 260a (HUAA); Mpio. Jesús María: Presa Los Arquitos, $21^{\circ} 55^{\prime} 23^{\prime \prime} \mathrm{N}, 102^{\circ} 23^{\prime} 20^{\prime \prime} \mathrm{W}, \mathrm{O}$. Rosales 2231 (HUAA); Mpio. San José de Gracia: Cañada Mesa Montoro, 2200’12" N,

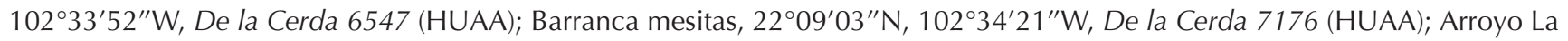
Mesa del Amo entre cerro de La Calavera y cerro El Colorín, 21 $58^{\prime} 32^{\prime \prime} \mathrm{N}, 102^{\circ} 30^{\prime} 56^{\prime \prime} \mathrm{W}$, Sierra-Muñoz 355 (HUAA); $11.7 \mathrm{~km}$ al SW de Rincón de Romos, $22^{\circ} 08^{\prime} 49^{\prime \prime} \mathrm{N}, 102^{\circ} 21^{\prime} 52^{\prime \prime} \mathrm{W}$, Sierra-Muñoz 557 (HUAA); 3 km al NW de la cortina de la presa El Jocoqui, $22^{\circ} 08^{\prime} 04^{\prime \prime} \mathrm{N}, 102^{\circ} 22^{\prime} 55^{\prime \prime} \mathrm{W}$, Sierra-Muñoz 562 (HUAA). Jaltomata procumbens. Mpio. Aguascalientes: Cerro de Los Gallos,

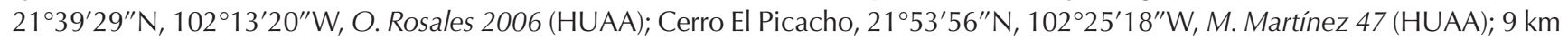
al SSE del cruce carretera 45 a San Bartolo, $21^{\circ} 43^{\prime} 50^{\prime \prime} \mathrm{N}, 102^{\circ} 11^{\prime} 06^{\prime \prime} \mathrm{W}$, Sierra-Muñoz 483 (HUAA); Ladera N del cerro Los GaIlos, $21^{\circ} 39^{\prime} 44^{\prime \prime} \mathrm{N}, 102^{\circ} 13^{\prime} 27^{\prime \prime} \mathrm{W}$, Sierra-Muñoz 802 (HUAA); Mpio. Calvillo: El Guarache, $15 \mathrm{~km}$ al NE del Terrero del Refugio,

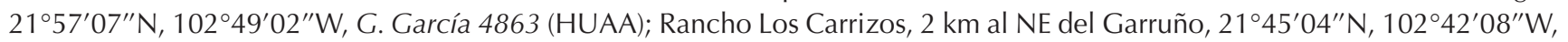

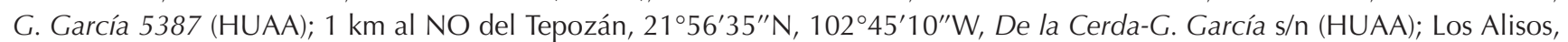

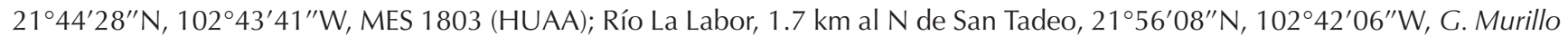

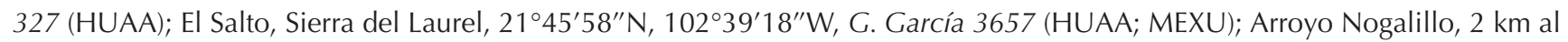

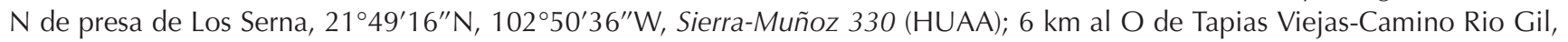

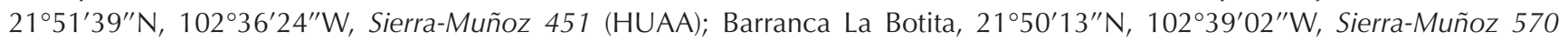
(HUAA); 600 m al SSE de El Sauz de La Labor, 21 $59^{\prime} 35^{\prime \prime} \mathrm{N}, 102^{\circ} 38^{\prime} 52^{\prime \prime} \mathrm{W}$, Sierra-Muñoz 837 (HUAA); El Salto, Sierra del Laurel,

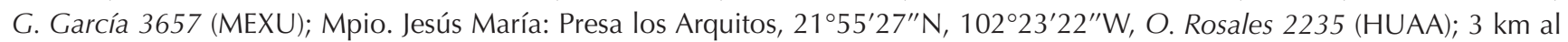

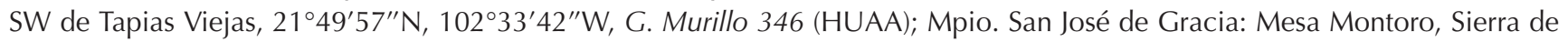

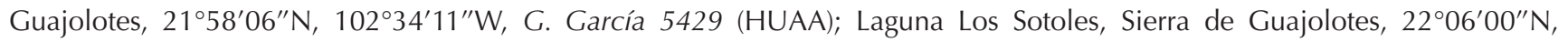

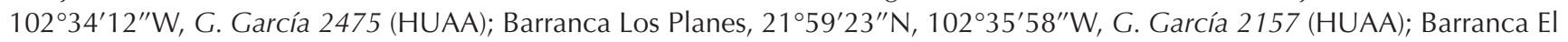

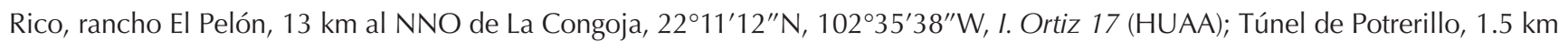
al E de Boca de Túnel de Potrerillo, 22 $2^{\circ} 4^{\prime} 04^{\prime \prime} \mathrm{N}, 102^{\circ} 25^{\prime} 31^{\prime \prime} \mathrm{W}$, Sierra-Muñoz 735 (HUAA); Ciénega de Gallardo, 22 $08^{\prime} 17^{\prime \prime} \mathrm{N}$, $102^{\circ} 34^{\prime} 51^{\prime \prime} \mathrm{W}$, Sierra-Muñoz 820 (HUAA); 1.7 km al NE de Ciénega de Gallardo, 22 $08^{\prime} 32^{\prime \prime} \mathrm{N}, 102^{\circ} 39^{\prime} 07^{\prime \prime} \mathrm{W}$, Sierra-Muñoz 828 (HUAA); Laguna de los Sotoles, Sierra de Guajolotes, G. García 2475 (IEB); Mpio. Tepezalá: NW de cerro Mesillas, 22¹9’57"N,

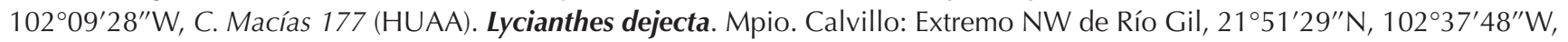
G. García 4274 (HUAA); 5 km al E del Sauz, 21 $53^{\prime} 04^{\prime \prime} \mathrm{N}, 102^{\circ} 33^{\prime} 54^{\prime \prime}$ W, G. García 4620 (HUAA); Ladera Norte del cerro de La Iguana, $21^{\circ} 54^{\prime} 58^{\prime \prime} \mathrm{N}, 102^{\circ} 38^{\prime} 29^{\prime \prime} \mathrm{W}$, J. Martínez 1757 (HUAA); $500 \mathrm{~m}$ al N de Barranca Obscura, 21 $48^{\prime} 02^{\prime \prime} \mathrm{N}, 102^{\circ} 38^{\prime} 38^{\prime \prime} \mathrm{W}$,

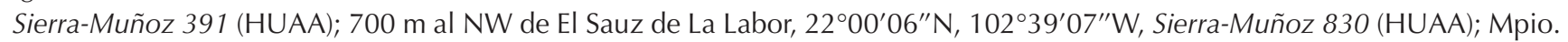

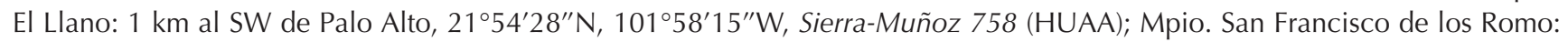
$2.3 \mathrm{~km}$ al E de Borrotes, $22^{\circ} 02^{\prime} 29^{\prime \prime} \mathrm{N}, 102^{\circ} 10^{\prime} 57^{\prime \prime} \mathrm{W}$, Sierra-Muñoz 797 (HUAA). Lycianthes moziniana var moziniana. Mpio.

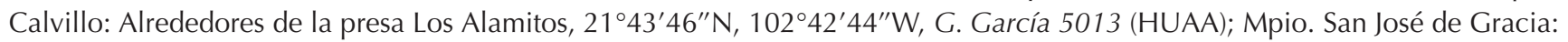
Barranca Revientacuartas, $14 \mathrm{~km}$ al SW de La Congoja, 22 $09^{\prime} 01^{\prime \prime} \mathrm{N}, 102^{\circ} 36^{\prime} 59^{\prime \prime} \mathrm{W}$, G. García 3931 (HUAA); Barranca Juan

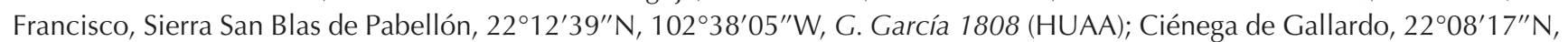
102 39'51"W, Sierra-Muñoz 821 (HUAA); 1 km al NW de La Congoja, 22¹0'23”N, 102³3'56”W, Sierra-Muñoz 892 (HUAA). Nicandra physalodes. Mpio. Calvillo: Barranca de Mesa Grande, G. García 5345 (HUAA); 2 km al E del Sauz, Barranca Cebolletas, Sierra-Muñoz 511 (HUAA). Nicotiana glauca. Mpio. Aguascalientes: Rancho "Los Cocuyos" km 7 carr. Aguascalientes - Ca-

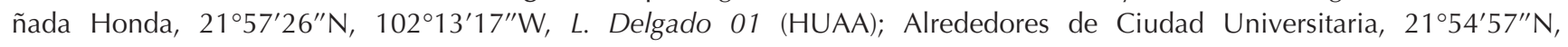
$102^{\circ} 19^{\prime} 10^{\prime \prime} \mathrm{W}$, Croce M. s/n (HUAA); $9 \mathrm{~km}$ al SE del cruce carretera 45 a San Bartolo, $21^{\circ} 45^{\prime} 04^{\prime \prime} \mathrm{N}, 102^{\circ} 12^{\prime} 47^{\prime \prime} \mathrm{W}$, Sierra-Muñoz 473 (HUAA); $9 \mathrm{~km}$ al SSE del cruce carretera 45 a San Bartolo, 21 $43^{\prime} 50^{\prime \prime} \mathrm{N}, 102^{\circ} 11^{\prime} 06^{\prime \prime} \mathrm{W}$, Sierra-Muñoz 480 (HUAA); 3 km al NE de Los Caños, $21^{\circ} 48^{\prime} 37^{\prime \prime} \mathrm{N}, 102^{\circ} 27^{\prime} 02^{\prime \prime} \mathrm{W}$, Sierra-Muñoz 599 (HUAA); Alrededores de la presa El Niagara, 2146 $49^{\prime \prime} \mathrm{N}$, 
José CARlos Sierra-MuÑoz ET AL.

Apéndice I. Continuación.

$102^{\circ} 21^{\prime} 56^{\prime \prime} \mathrm{W}$, Sierra-Muñoz 611 (HUAA); Mpio. Asientos: $3 \mathrm{~km}$ al N de El Llavero, $22^{\circ} 04^{\prime} 08^{\prime \prime} \mathrm{N}, 102^{\circ} 05^{\prime} 59^{\prime \prime} \mathrm{W}$, Sierra-Muñoz $^{\circ}$ 430 (HUAA); Bordo al extremo SW de Plutarco Elías Calles, 22 ${ }^{\circ} 12^{\prime} 32^{\prime \prime} \mathrm{N}, 102^{\circ} 03^{\prime} 25^{\prime \prime} \mathrm{W}, \mathrm{l}$. Ortiz 05 (HUAA); 2 km al SE de Asientos, $22^{\circ} 13^{\prime} 38^{\prime \prime} \mathrm{N}, 102^{\circ} 04^{\prime} 28^{\prime \prime} \mathrm{W}$, Sierra-Muñoz 649 (HUAA); $2 \mathrm{~km}$ al S de Asientos, 22 ${ }^{\circ} 13^{\prime} 15^{\prime \prime} \mathrm{N}, 102^{\circ} 05^{\prime} 11^{\prime \prime} \mathrm{W}$, Sierra-Muñoz 651 (HUAA); 2 km al NE de Asientos, 22 ${ }^{\circ} 14^{\prime} 45^{\prime \prime} \mathrm{N}, 102^{\circ} 04^{\prime} 18^{\prime \prime} \mathrm{W}$, Sierra-Muñoz 689 (HUAA); $500 \mathrm{~m}$ al E de Puerto de la Concepción, $22^{\circ} 12^{\prime} 10^{\prime \prime} \mathrm{N}, 102^{\circ} 07^{\prime} 52^{\prime \prime} \mathrm{W}$, Sierra-Muñoz 765 (HUAA); W de Ojo de Agua de Los Sauces a 3 km al SE de Pilotos,

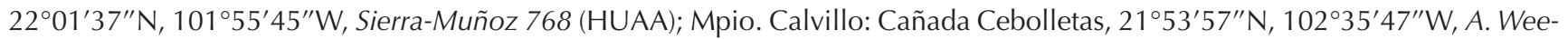

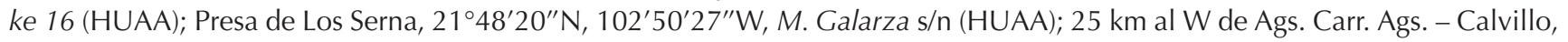

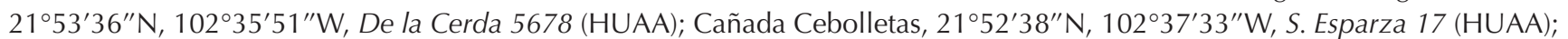
Presa La Codorniz, $21^{\circ} 59^{\prime} 41^{\prime \prime} \mathrm{N}, 102^{\circ} 29^{\prime} 58^{\prime \prime} \mathrm{W}$, R. Tiscareño s/n (HUAA); $6 \mathrm{~km}$ al E del Sauz, $21^{\circ} 54^{\prime} 21^{\prime \prime} \mathrm{N}, 102^{\circ} 34^{\prime} 57^{\prime \prime} \mathrm{W}$, Sierra- $^{\circ}$ Muñoz 412 (HUAA); 8 km al SW de Tapias Viejas, 21 $51^{\prime} 52^{\prime \prime} \mathrm{N}, 102^{\circ} 37^{\prime} 16^{\prime \prime} \mathrm{W}$, Sierra-Muñoz 464 (HUAA); 3 km al SW del Sauz,

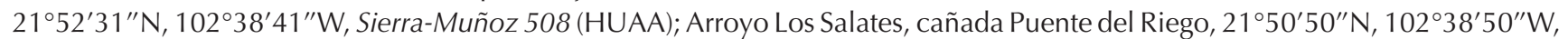

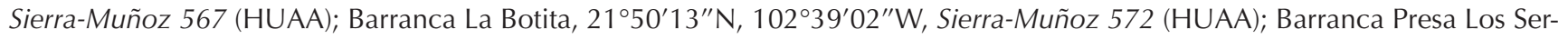

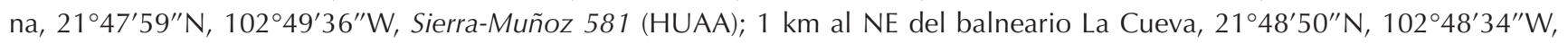
Sierra-Muñoz 621 (HUAA); Barranca Las Moras, $1 \mathrm{~km}$ al SE de la cortina de la presa Las Moras, $21^{\circ} 46^{\prime} 06^{\prime \prime} \mathrm{N}, 102^{\circ} 42^{\prime} 52^{\prime \prime} \mathrm{W}$,

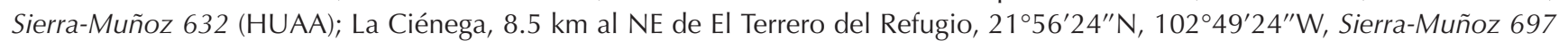
(HUAA); 2 km al NE de Malpaso, 21 ${ }^{\circ} 52^{\prime} 23^{\prime \prime} \mathrm{N}, 102^{\circ} 39^{\prime} 22^{\prime \prime} \mathrm{W}$, Sierra-Muñoz 715 (HUAA); Márgenes de la Presa La Ordeña Vieja, 21 $56^{\prime} 53^{\prime \prime} \mathrm{N}, 102^{\circ} 43^{\prime} 14^{\prime \prime} \mathrm{W}$, G. Murillo 314 (HUAA); $1 \mathrm{~km}$ al E de El Zapote, $21^{\circ} 58^{\prime} 18^{\prime \prime} \mathrm{N}, 102^{\circ} 38^{\prime} 59^{\prime \prime} \mathrm{W}$, Sierra-Muñoz 840

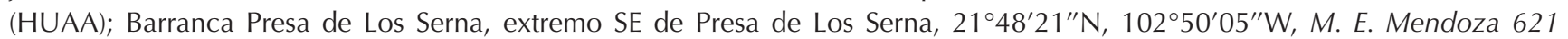
(HUAA); Barranca Cebolletas, $2 \mathrm{~km}$ al W de El Sauz, 21 $53^{\prime} 16^{\prime \prime} \mathrm{N}, 102^{\circ} 35^{\prime} 46^{\prime \prime} \mathrm{W}$, H. Araiza 406 (HUAA); Mpio. Cosío: $1.2 \mathrm{~km}$

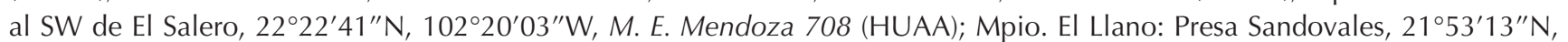

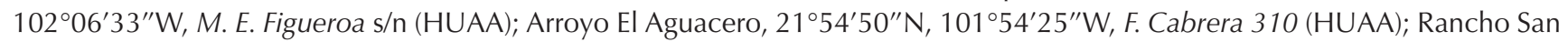
Sebastián a $4.7 \mathrm{~km}$ al SE de Calvillito, $21^{\circ} 50^{\prime} 10^{\prime \prime} \mathrm{N}, 102^{\circ} 08^{\prime} 30^{\prime \prime} \mathrm{W}$, Sierra-Muñoz 748 (HUAA); $1.2 \mathrm{~km}$ al SW de Los Caños,

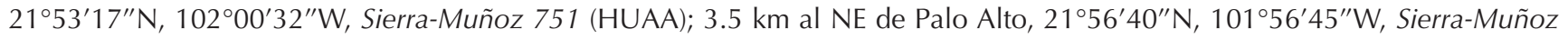

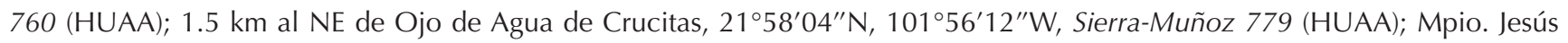
María: $4.5 \mathrm{~km}$ al SW de Jesús María, 21 ${ }^{\circ} 57^{\prime} 47^{\prime \prime} \mathrm{N}, 102^{\circ} 23^{\prime} 18^{\prime \prime} \mathrm{W}$, Sierra-Muñoz 546 (HUAA); Barranca a 4 Km al N de Milpillas,

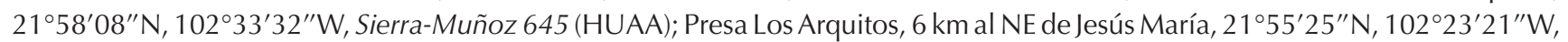

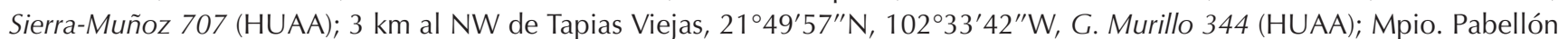
de Arteaga: Arroyo Santiago, 2 km al W de Santiago, $22^{\circ} 07^{\prime} 17^{\prime \prime} \mathrm{N}, 102^{\circ} 21^{\prime} 19^{\prime \prime} \mathrm{W}, \mathrm{M}$. E. Mendoza 585 (HUAA); Mpio. Rincón de Romos: Ajiladero, $22^{\circ} 17^{\prime} 23^{\prime \prime} \mathrm{N}, 102^{\circ} 21^{\prime} 46^{\prime \prime} \mathrm{W}, \mathrm{N}$. Arroyo 27 (HUAA); Arroyo La Boquilla a $500 \mathrm{~m}$ al W de La Boquilla, $22^{\circ} 15^{\prime} 50^{\prime \prime} \mathrm{N}, 102^{\circ} 22^{\prime} 37^{\prime \prime} \mathrm{W}$, J. Martínez 2501 (HUAA); $3.5 \mathrm{~km}$ al W de La Punta, 22 ${ }^{\circ} 19^{\prime} 07^{\prime \prime} \mathrm{N}, 102^{\circ} 19^{\prime} 41^{\prime \prime} \mathrm{W}$, Sierra-Muñoz 855 (HUAA); Arroyo del Ejido Morelos, 4.2 km al NNW de Rincón de Romos, $22^{\circ} 14^{\prime} 12^{\prime \prime} \mathrm{N}, 102^{\circ} 21^{\prime} 40^{\prime \prime} \mathrm{W}$, M. E. Mendoza 567 (HUAA); Mpio. San Francisco de los Romo: 1.5 km al NW de Borrotes, 22 ${ }^{\circ} 02^{\prime} 34^{\prime \prime} \mathrm{N}, 102^{\circ} 13^{\prime} 04^{\prime \prime}$ W, Sierra-Muñoz 790 (HUAA); Mpio. San José de Gracia: Entronque a la Cieneguita, Carretera 19 estatal, $21^{\circ} 10^{\prime} 12^{\prime \prime} \mathrm{N}, 102^{\circ} 26^{\prime} 20^{\prime \prime} \mathrm{W}, \mathrm{C}$. D. Alba 50 (HUAA);

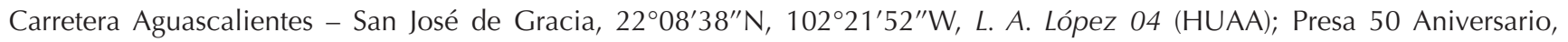
$22^{\circ} 11^{\prime} 36^{\prime \prime} \mathrm{N}, 102^{\circ} 28^{\prime} 11^{\prime \prime} \mathrm{W}$, E. Padilla s/n (HUAA); $11.75 \mathrm{~km}$ al NO de Rincón de Romos, $22^{\circ} 08^{\prime} 49^{\prime \prime} \mathrm{N}, 1^{\circ} 02^{\circ} 21^{\prime} 52^{\prime \prime} \mathrm{W}$, Sierra- $^{\prime}$ Muñoz 555 (HUAA); 3 km al NW de la cortina de la presa El Jocoqui, 22 $08^{\prime} 04^{\prime \prime} \mathrm{N}, 102^{\circ} 22^{\prime} 55^{\prime \prime} \mathrm{W}$, Sierra-Muñoz 563 (HUAA); $700 \mathrm{~m}$ al W de la cortina de la presa Calles, $22^{\circ} 08^{\prime} 17^{\prime \prime} \mathrm{N}, 102^{\circ} 24^{\prime} 47^{\prime \prime} \mathrm{W}, \mathrm{M}$. E. Mendoza 431 (HUAA); Cañada a $500 \mathrm{~m}$ al SE de rancho Viejo, $22^{\circ} 07^{\prime} 17^{\prime \prime} \mathrm{N}, 102^{\circ} 30^{\prime} 38^{\prime \prime} \mathrm{W}$, G. Murillo 272 (HUAA); Barranca El Carrizal, $4.5 \mathrm{~km}$ al NW de San Antonio de los Ríos, $22^{\circ} 11^{\prime} 05^{\prime \prime} \mathrm{N}, 102^{\circ} 30^{\prime} 23^{\prime \prime} \mathrm{W}$, Sierra-Muñoz 681 (HUAA); Boca de Túnel de Potrerillo, 22 ${ }^{\circ} 14^{\prime} 34^{\prime \prime} \mathrm{N}, 1^{\circ} 2^{\circ} 26^{\prime} 43^{\prime \prime} \mathrm{W}$, SierraMuñoz 729 (HUAA); Cortina de la presa 50 Aniversario, $22^{\circ} 11^{\prime} 04^{\prime \prime} \mathrm{N}, 102^{\circ} 27^{\prime} 53^{\prime \prime} \mathrm{W}$, Sierra-Muñoz 744 (HUAA); Margen NW de la presa Calles, $22^{\circ} 09^{\prime} 42^{\prime \prime} \mathrm{N}, 102^{\circ} 27^{\prime} 22^{\prime \prime} \mathrm{W}, \mathrm{M}$. E. Mendoza 545 (HUAA); Mpio. Tepezalá: Arroyo Las Pilas, 22 ${ }^{\circ} 15^{\prime} 32^{\prime \prime} \mathrm{N}$, $102^{\circ} 10^{\prime} 18^{\prime \prime} \mathrm{W}$, F. cabrera 286 (HUAA); Presa Mesillas, $1 \mathrm{~km}$ al SE de Mesillas, $22^{\circ} 18^{\prime} 43^{\prime \prime} \mathrm{N}, 102^{\circ} 09^{\prime} 40^{\prime \prime} \mathrm{W}$, Sierra-Muñoz 668 (HUAA); $7.4 \mathrm{~km}$ al NW de Tepezalá, $22^{\circ} 13^{\prime} 41^{\prime \prime} \mathrm{N}, 102^{\circ} 11^{\prime} 10^{\prime \prime} \mathrm{W}$, Sierra-Muñoz 673 (HUAA); $2.7 \mathrm{~km}$ al SE de Tepezalá, $22^{\circ} 15^{\prime} 50^{\prime \prime} \mathrm{N}, 102^{\circ} 09^{\prime} 07^{\prime \prime} \mathrm{W}$, Sierra-Muñoz 676 (HUAA); 4 km al E de Tepezalá, 22 ${ }^{\circ} 13^{\prime} 13^{\prime \prime} \mathrm{N}, 102^{\circ} 08^{\prime} 06^{\prime \prime} \mathrm{W}$, Sierra-Muñoz 687 (HUAA). Nicotiana obtusifolia. Mpio. Aguascalientes: Camino presa El Maguey, $21^{\circ} 45^{\prime} 27^{\prime \prime} \mathrm{N}, 102^{\circ} 25^{\prime} 58^{\prime \prime} \mathrm{W}$, De la Cerda 7015 (HUAA); Alrededores del Gachupín, 21 $51^{\prime} 48^{\prime \prime} \mathrm{N}, 102^{\circ} 14^{\prime} 14^{\prime \prime} \mathrm{W}$, De la Cerda 1735 (HUAA); Arroyo de Paso Hondo, $21^{\circ} 51^{\prime} 22^{\prime \prime} \mathrm{N}$,

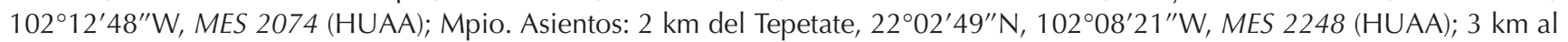
$\mathrm{N}$ de El Llavero, $22^{\circ} 04^{\prime} 08^{\prime \prime} \mathrm{N}, 102^{\circ} 05^{\prime} 59^{\prime \prime} \mathrm{W}$, Sierra-Muñoz 429 (HUAA); NE de Mesa Mesillas, 22 ${ }^{\circ} 19^{\prime} 54^{\prime \prime} \mathrm{N}, 102^{\circ} 09^{\prime} 23^{\prime \prime} \mathrm{W}, \mathrm{G}$.

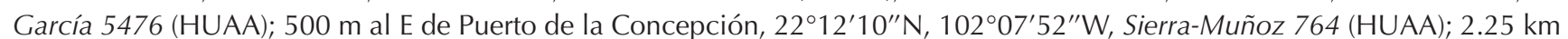

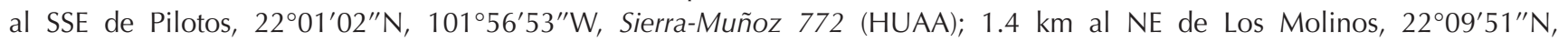

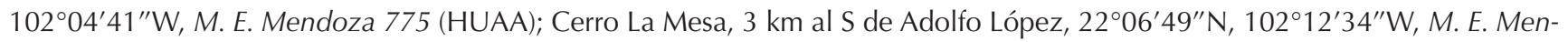


Solanaceae en Aguascalientes, México

Apéndice I. Continuación.

doza 483 (HUAA); 2 km al S de Asientos, 22 ${ }^{\circ} 13^{\prime} 15^{\prime \prime} \mathrm{N}, 102^{\circ} 05^{\prime} 11^{\prime \prime} \mathrm{W}$, Sierra-Muñoz 654 (HUAA); Mpio. Calvillo: Sierra del

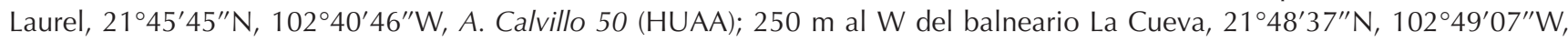

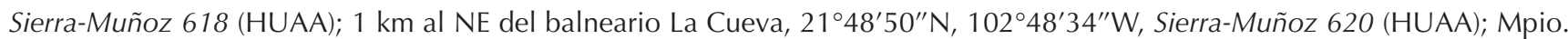

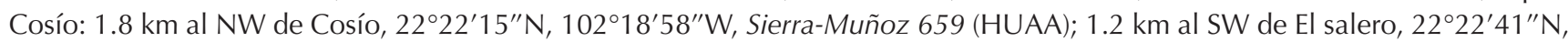
$102^{\circ} 20^{\prime} 03^{\prime \prime} \mathrm{W}$, M. E. Mendoza 709 (HUAA); $1.5 \mathrm{~km}$ al NE de Ojo de Agua de Crucitas, $21^{\circ} 58^{\prime} 04^{\prime \prime} \mathrm{N}, 101^{\circ} 56^{\prime} 12^{\prime \prime} \mathrm{W}$, Sierra-Muñoz 781 (HUAA); Mpio. Pabellón de Arteaga: Faldas del cerro del Chiquihuite, 22 $06^{\prime} 24^{\prime \prime} \mathrm{N}, 102^{\circ} 13^{\prime} 24^{\prime \prime} \mathrm{W}$, O. Rosales \& Croce 206 (HUAA); Mpio. Rincón de Romos: Arroyo La Boquilla a 500 m al W de La Boquilla, 22 $15^{\prime} 50^{\prime \prime} \mathrm{N}, 102^{\circ} 22^{\prime} 37^{\prime \prime} \mathrm{W}$, J. Martínez 2500 (HUAA); Arroyo del Ejido Morelos a $4.5 \mathrm{~km}$ al NNW de Rincón de Romos, $22^{\circ} 14^{\prime} 12^{\prime \prime} \mathrm{N}, 102^{\circ} 21^{\prime} 40^{\prime \prime} \mathrm{W}$, J. Martínez 2490

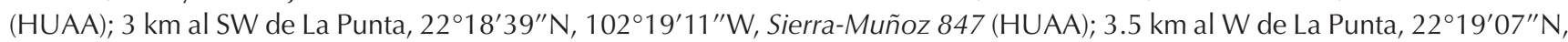
$102^{\circ} 19^{\prime} 41^{\prime \prime} \mathrm{W}$, Sierra-Muñoz 854 (HUAA); 2 km al E de Borrotes, $22^{\circ} 02^{\prime} 04^{\prime \prime} \mathrm{N}, 102^{\circ} 11^{\prime} 14^{\prime \prime} \mathrm{W}$, Sierra-Muñoz 785 (HUAA); 2.3 km

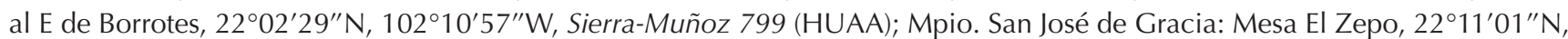

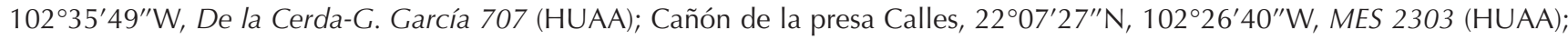

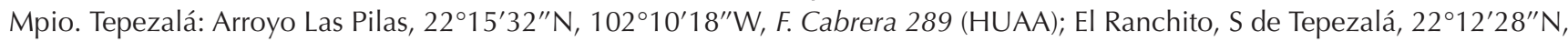
$102^{\circ} 10^{\prime} 02^{\prime \prime} \mathrm{W}$, De la Cerda 7126 (HUAA); $7.4 \mathrm{~km}$ al NW de Tepezalá, 22 $12^{\prime} 41^{\prime \prime} \mathrm{N}, 102^{\circ} 11^{\prime} 10^{\prime \prime}$ W, Sierra-Muñoz 672 (HUAA); $2.7 \mathrm{~km}$ al SE de Tepezalá, 22 ${ }^{\circ} 15^{\prime} 50^{\prime \prime} \mathrm{N}, 102^{\circ} 09^{\prime} 07^{\prime \prime} \mathrm{W}$, Sierra-Muñoz 675 (HUAA); Arroyo Las Pilas, 2 km al NW de la exhacienda Las Pilas, $22^{\circ} 15^{\prime} 59^{\prime \prime} \mathrm{N}, 102^{\circ} 10^{\prime} 50^{\prime \prime} \mathrm{W}$, M. E. Mendoza 740 (HUAA); Arroyo Las Pilas, $1.3 \mathrm{~km}$ al NW de la exhacienda Las Pilas, $22^{\circ} 15^{\prime} 43^{\prime \prime} \mathrm{N}, 102^{\circ} 10^{\prime} 32^{\prime \prime} \mathrm{W}$, M. E. Mendoza 483 (HUAA). Nicotiana plumbaginifolia. Mpio. Aguascalientes: Coto San Nicolás, $21^{\circ} 51^{\prime} 06^{\prime \prime} \mathrm{N}, 102^{\circ} 21^{\prime} 20^{\prime \prime} \mathrm{W}$, S. Villalpando 09 (HUAA); Alrededores de la presa El Niágara, $21^{\circ} 46^{\prime} 49^{\prime \prime} \mathrm{N}, 102^{\circ} 21^{\prime} 56^{\prime \prime} \mathrm{W}$,

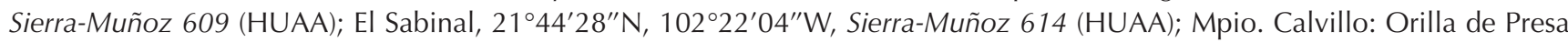
Los Serna, $21^{\circ} 48^{\prime} 12^{\prime \prime} \mathrm{N}, 102^{\circ} 50^{\prime} 46^{\prime \prime} \mathrm{W}$, De la Cerda 6635 (HUAA; IEB). Nicotiana tabacum. Mpio. Calvillo: Barranca Presa Los Serna, De la cerda 7397 (HUAA); Barranca obscura, Sierra del Laurel, K. Landín 151 (HUAA); Barranca La Botita, $3 \mathrm{~km}$ al SE de Malpaso, Sierra-Muñoz 590 (HUAA). Nierembergia angustifolia. Mpio. Aguascalientes: Rancho La Aurora, 17 km al E de Aguascalientes, $21^{\circ} 48^{\prime} 58^{\prime \prime} \mathrm{N}, 102^{\circ} 05^{\prime} 52^{\prime \prime} \mathrm{W}$, De la Cerda 5899 (HUAA; IEB); Presa de San Bartolo, $21^{\circ} 44^{\prime} 38^{\prime \prime} \mathrm{N}, 102^{\circ} 09^{\prime} 14^{\prime \prime} \mathrm{W}, \mathrm{MES}^{\circ}$ 2133 (HUAA); Bordo secundario de la presa San Bartolo, 21 ${ }^{\circ} 44^{\prime} 28^{\prime \prime} \mathrm{N}, 102^{\circ} 07^{\prime} 04^{\prime \prime} \mathrm{W}, \mathrm{M}$. Palacio 22 (HUAA); Presa Las Grullas,

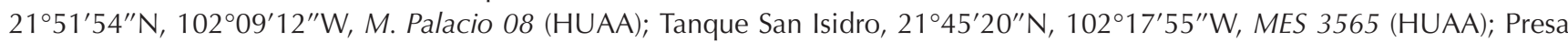
del Muerto, $21^{\circ} 44^{\prime} 10^{\prime \prime} \mathrm{N}, 102^{\circ} 13^{\prime} 39^{\prime \prime} \mathrm{W}$, MES 3642 (HUAA); $500 \mathrm{~m}$ al N de Los Caños, $21^{\circ} 47^{\prime} 30^{\prime \prime} \mathrm{N}, 1^{\circ} 2^{\circ} 28^{\prime} 09^{\prime \prime} \mathrm{W}, \mathrm{G}^{\prime}$. Murillo

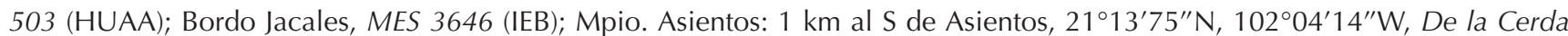
6060 (HUAA); Bordo temporal de Molino Viejo, km 3 carr. Villa Juarez-Asientos, $22^{\circ} 08^{\prime} 34^{\prime \prime} \mathrm{N}, 102^{\circ} 02^{\prime} 17^{\prime \prime} \mathrm{W}, \mathrm{MES} 3610$ (HUAA); Presa El Llavero, $22^{\circ} 04^{\prime} 00^{\prime \prime} \mathrm{N}, 102^{\circ} 05^{\prime} 48^{\prime \prime} \mathrm{W}$, Sierra-Muñoz 432 (HUAA); Bordo al extremo SW de Plutarco Elías Calles,

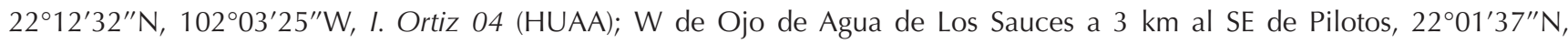
$101^{\circ} 55^{\prime} 45^{\prime \prime} \mathrm{W}$, Sierra-Muñoz 769 (HUAA); 4 km al SSE de Pilotos, 22 $00^{\prime} 21^{\prime \prime} \mathrm{N}, 101^{\circ} 56^{\prime} 26^{\prime \prime} \mathrm{W}$, Sierra-Muñoz 777 (HUAA); Mpio. Cosío: Bordo Zacatequillas al E del poblado Zacatequillas, $22^{\circ} 25^{\prime} 54^{\prime \prime} \mathrm{N}, 102^{\circ} 18^{\prime} 11^{\prime \prime} \mathrm{W}$, O. Rosales 3075 (HUAA); Presa chica

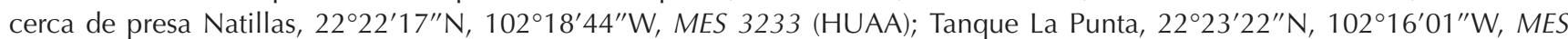

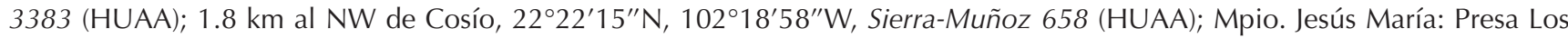

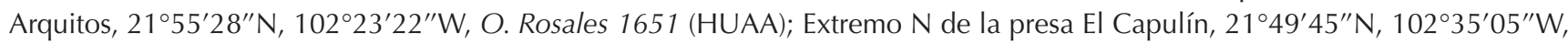

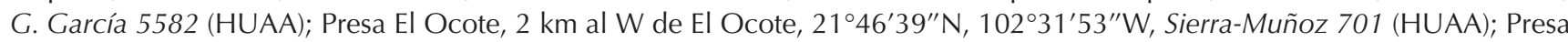
El Capulín, 9 km al NW de El Ocote, $21^{\circ} 49^{\prime} 43^{\prime \prime} \mathrm{N}, 102^{\circ} 35^{\prime} 07^{\prime \prime}$ W, Sierra-Muñoz 706 (HUAA); Presa Los Arquitos, 6 km al NE de

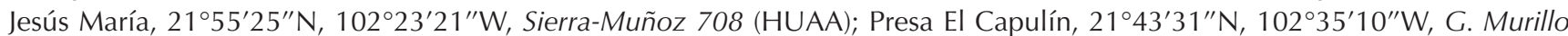

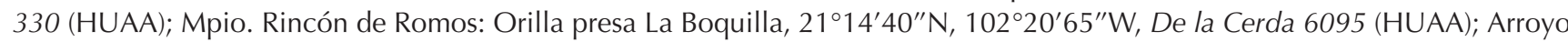

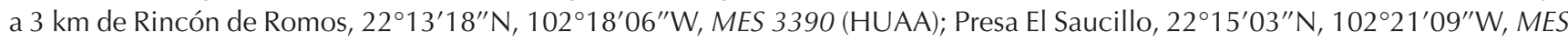
3244 (HUAA); Arroyo del Ejido Morelos, 4.2 km al NNW de Rincón de Romos, 22 ${ }^{\circ} 14^{\prime} 12^{\prime \prime} \mathrm{N}, 102^{\circ} 21^{\prime} 40^{\prime \prime} \mathrm{W}$, M. E. Mendoza 565 (HUAA); Márgenes de la presa de Escaleras, $2.5 \mathrm{~km}$ al NW de Rincón de Romos, $22^{\circ} 14^{\prime} 32^{\prime \prime} \mathrm{N}, 102^{\circ} 20^{\prime} 33^{\prime \prime} \mathrm{W}$, M. E. Mendoza 562 (HUAA); Mpio. San José de Gracia: Presa Calles, 22 ${ }^{\circ} 09^{\prime} 00^{\prime \prime} \mathrm{N}, 102^{\circ} 25^{\prime} 18^{\prime \prime} \mathrm{W}$, Sierra-Muñoz 373 (HUAA); 3 km al NW de la

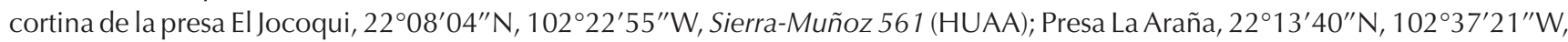
Sierra-Muñoz 713 (HUAA); Cortina de la presa 50 Aniversario, 22 ${ }^{\circ} 11^{\prime} 04^{\prime \prime} \mathrm{N}, 102^{\circ} 27^{\prime} 53^{\prime \prime} \mathrm{W}$, Sierra-Muñoz 741 (HUAA); Margen NW de la presa Calles, $22^{\circ} 09^{\prime} 42^{\prime \prime} \mathrm{N}, 102^{\circ} 27^{\prime} 22^{\prime \prime} \mathrm{W}$, M. E. Mendoza 540 (HUAA). Petunia parviflora. Mpio. Aguascalientes: Jar-

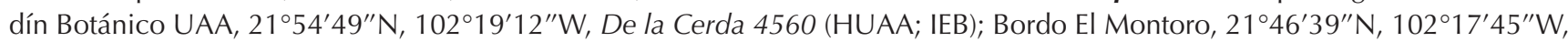
MES 3560 (HUAA); Presa El Muerto al E de peñuelas, 21 ${ }^{\circ} 43^{\prime} 08^{\prime \prime} \mathrm{N}, 102^{\circ} 13^{\prime} 06^{\prime \prime} \mathrm{W}$, O. Rosales 1341 (HUAA); Alrededores de la

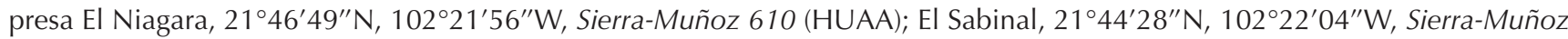
613 (HUAA); Mpio. Asientos: $1 \mathrm{~km}$ al S de Asientos, 22 ${ }^{\circ} 13^{\prime} 48^{\prime \prime} \mathrm{N}, 102^{\circ} 05^{\prime 2} 23^{\prime \prime} \mathrm{W}$, De la Cerda 6061 (HUAA); Presa El Llavero, $22^{\circ} 04^{\prime} 00^{\prime \prime} \mathrm{N}, 102^{\circ} 05^{\prime} 48^{\prime \prime} \mathrm{W}$, Sierra-Muñoz 434 (HUAA); $2 \mathrm{~km}$ al S de Asientos, $22^{\circ} 13^{\prime} 15^{\prime \prime} \mathrm{N}, 102^{\circ} 05^{\prime} 11^{\prime \prime} \mathrm{W}$, Sierra-Muñoz 655 
José CARlos Sierra-MuÑoz ET AL.

Apéndice I. Continuación.

(HUAA); $2 \mathrm{~km}$ al NE de Asientos, $22^{\circ} 14^{\prime} 45^{\prime \prime} \mathrm{N}, 102^{\circ} 04^{\prime} 18^{\prime \prime} \mathrm{W}$, Sierra-Muñoz 690 (HUAA); Barranca Presa de Los Serna,

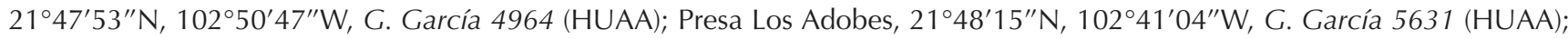
$250 \mathrm{~m}$ al W del balneario La Cueva, 21 ${ }^{\circ} 48^{\prime} 37^{\prime \prime} \mathrm{N}, 102^{\circ} 49^{\prime} 07^{\prime \prime} \mathrm{W}$, Sierra-Muñoz 617 (HUAA); $1 \mathrm{~km}$ al NE del balneario La Cueva,

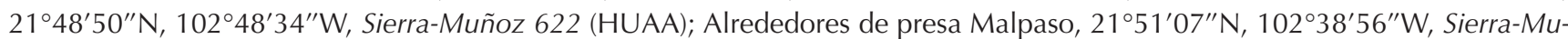
ñoz 722 (HUAA); $2.25 \mathrm{~km}$ al SSE de Pilotos, $22^{\circ} 01^{\prime} 02^{\prime \prime} \mathrm{N}, 101^{\circ} 56^{\prime} 53^{\prime \prime} \mathrm{W}$, Sierra-Muñoz 773 (HUAA); W de Ojo de Agua de Los Sauces a $3 \mathrm{~km}$ al SE de Pilotos, $22^{\circ} 01^{\prime} 37^{\prime \prime} \mathrm{N}, 1^{\circ}{ }^{\circ} 55^{\prime} 45^{\prime \prime} \mathrm{W}$, Sierra-Muñoz 770 (HUAA); Mpio. Cosío: Presa chica cerca de presa

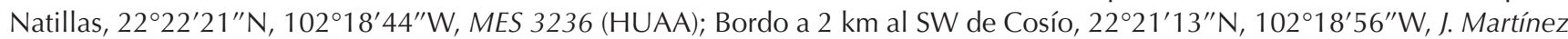
2261 (HUAA); $1.8 \mathrm{~km}$ al NW de Cosío, 22 $2^{\circ} 2^{\prime} 15^{\prime \prime} \mathrm{N}, 102^{\circ} 18^{\prime} 58^{\prime \prime} \mathrm{W}$, Sierra-Muñoz 661 (HUAA); Presa El Ocote, 2 km al W de El

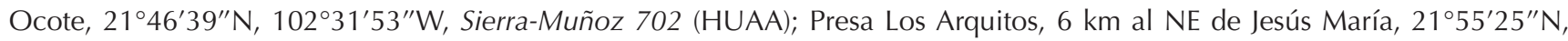

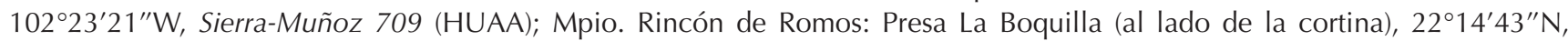
$102^{\circ} 22^{\prime} 37^{\prime \prime} \mathrm{W}$, O. Rosales 3081 (HUAA); La Sangría, 22 $2^{\circ} 13^{\prime} 00^{\prime \prime} \mathrm{N}, 102^{\circ} 17^{\prime} 21^{\prime \prime} \mathrm{W}$, O. Rosales 1825 (HUAA); Presa El Saucillo, $22^{\circ} 15^{\prime} 01^{\prime \prime} \mathrm{N}, 102^{\circ} 21^{\prime} 13^{\prime \prime} \mathrm{W}, \mathrm{MES} 3243$ (HUAA); Arroyo Ejido Morelos, $4.2 \mathrm{~km}$ al NNW de Rincón de Romos, 22 $2^{\circ} 4^{\prime} 12^{\prime \prime} \mathrm{N}$, $102^{\circ} 21^{\prime} 40^{\prime \prime}$ W, M. E. Mendoza 566 (HUAA); Márgenes de la presa de Escaleras, 2.5 km al NW de Rincón de Romos, 22¹4’32”N, 102²0’33"W, M. E. Mendoza 563 (HUAA); Presa La Boquilla, al lado de la cortina, O. Rosales 3081 (INEGI); Mpio. San José de Gracia: Presa Calles, $22^{\circ} 09^{\prime} 00^{\prime \prime} \mathrm{N}, 102^{\circ} 25^{\prime} 18^{\prime \prime} \mathrm{W}$, Sierra-Muñoz 374 (HUAA); $3 \mathrm{~km}$ al N de San José de Gracia, carretera a La Congoja, $22^{\circ} 10^{\prime} 25^{\prime \prime} \mathrm{N}, 102^{\circ} 25^{\prime} 10^{\prime \prime} \mathrm{W}$, Sierra-Muñoz 727 (HUAA); Margen NW de la presa Calles, 22 $2^{\circ} 09^{\prime} 42^{\prime \prime} \mathrm{N}, 102^{\circ} 27^{\prime} 22^{\prime \prime} \mathrm{W}$, M. E. Mendoza 541 (HUAA); Mpio. Tepezalá: 2.7 km al SE de Tepezalá, 22 ${ }^{\circ} 15^{\prime} 50^{\prime \prime} \mathrm{N}, 102^{\circ} 09^{\prime} 07^{\prime \prime}$ W, Sierra-Muñoz 679 (HUAA).

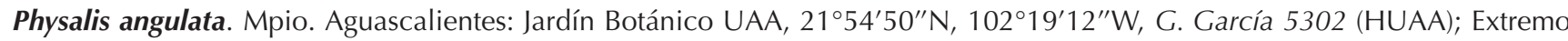
NW de la UAA, $21^{\circ} 54^{\prime} 16^{\prime \prime} \mathrm{N}, 102^{\circ} 19^{\prime} 3.4^{\prime \prime} \mathrm{W}$, G. García 5426 (HUAA); 1 km al S del rancho El Catorce, carr. Ags-Villa Hidalgo, $21^{\circ} 47^{\prime} 56^{\prime \prime} \mathrm{N}, 102^{\circ} 26^{\prime} 48^{\prime \prime} \mathrm{W}$, De la Cerda 5793 (HUAA); $1 \mathrm{~km}$ al SE de Tanque de Los Jiménez, $21^{\circ} 42^{\prime} 03^{\prime \prime} \mathrm{N}, 1^{\circ} 2^{\circ} 21^{\prime} 57^{\prime \prime} \mathrm{W}, \mathrm{Sie}^{\circ}$

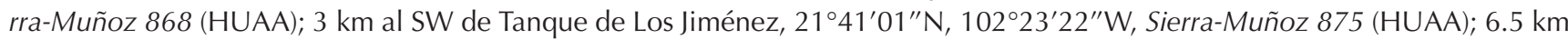

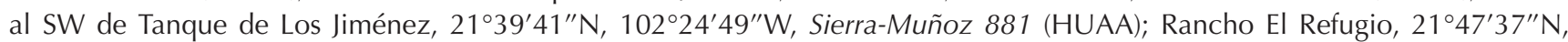
$102^{\circ} 15^{\prime} 10^{\prime \prime} \mathrm{W}$, Sierra-Muñoz 898 (HUAA); Mpio. Calvillo: Crucero Cerro Blanco-presa de Los Serna, 21 ${ }^{\circ} 50^{\prime} 20^{\prime \prime} \mathrm{N}, 1^{\circ} 2^{\circ} 48^{\prime} 28^{\prime \prime} \mathrm{W}$,

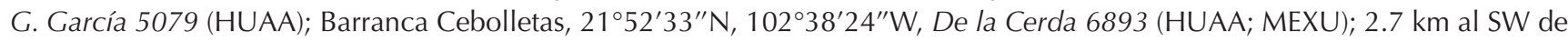

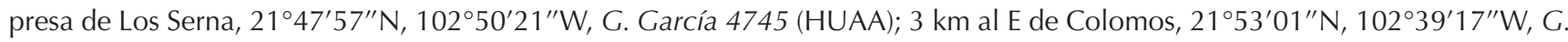

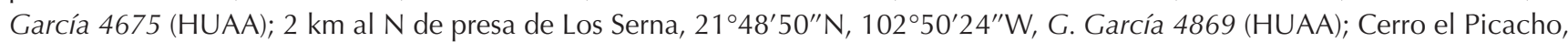

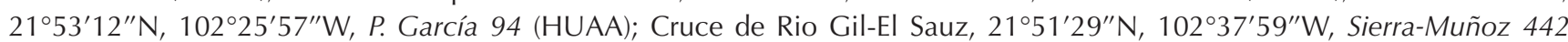

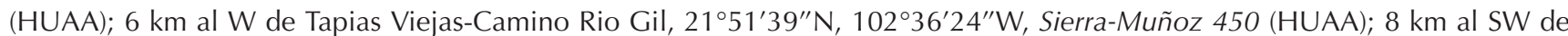

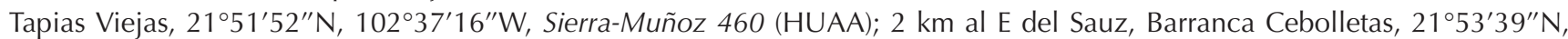

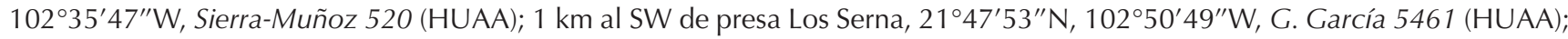

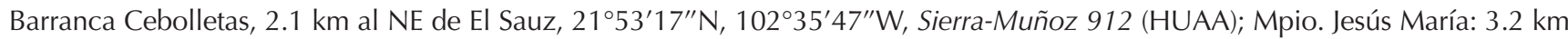
al NW de Gracias a Dios, $21^{\circ} 56^{\prime} 50^{\prime \prime} \mathrm{N}, 102^{\circ} 30^{\prime} 59^{\prime \prime} \mathrm{W}, \mathrm{M}$. E. Mendoza 695 (HUAA); $1.6 \mathrm{~km}$ al SE de Gracias a Dios, 21 ${ }^{\circ} 55^{\prime} 23^{\prime \prime} \mathrm{N}$, 102 $28^{\prime} 36^{\prime \prime}$ W, M. E. Mendoza 686 (HUAA). Physalis chenopodifolia. Mpio. Aguascalientes: 3 km al SW de Tanque de Los Jiménez, $21^{\circ} 41^{\prime} 01^{\prime \prime} \mathrm{N}, 102^{\circ} 23^{\prime} 22^{\prime \prime} \mathrm{W}$, Sierra-Muñoz 874 (HUAA); Mpio. Asientos: $2.25 \mathrm{~km}$ al SSE de Pilotos, $22^{\circ} 01^{\prime} 02^{\prime \prime} \mathrm{N}$,

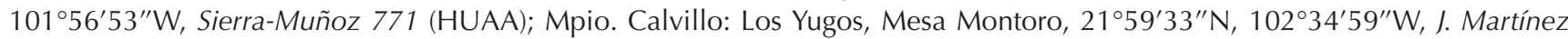

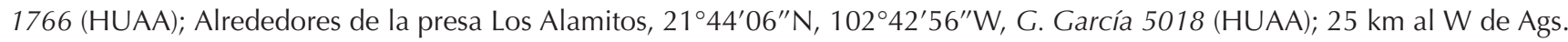

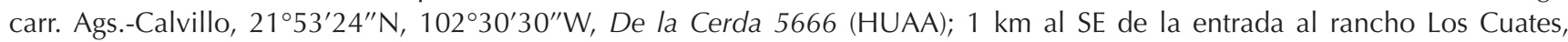

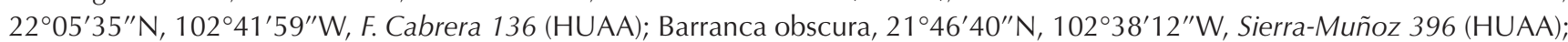
$6 \mathrm{~km}$ al SE de Jaltiche de Arriba, $21^{\circ} 44^{\prime} 31^{\prime \prime} \mathrm{N}, 102^{\circ} 45^{\prime} 00^{\prime \prime} \mathrm{W}$, Sierra-Muñoz 491 (HUAA); Mpio. El Llano: $1 \mathrm{~km}$ al SW de Palo

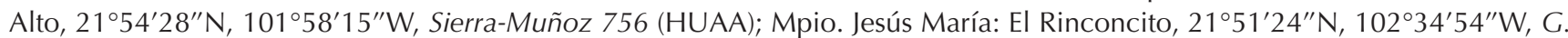
García 4636 (HUAA); Mpio. San José de Gracia: Sierra Fría, parque natural, 1/2 km antes de llegar a La Congoja, por carretera San José de Gracia-La Congoja, O. Vargas 773 (IBUG); Mesa El Águila, Sierra de San Blas de Pabellón, 22 ${ }^{\circ} 11^{\prime} 15^{\prime \prime} \mathrm{N}, 102^{\circ} 33^{\prime} 10^{\prime \prime} \mathrm{W}$, G. García 2243 (HUAA); Laguna Los Sotoles, barranca Montoro, 2205'26”N , 102³0'57"W, G. García 2491 (HUAA); Estación Biológica Agua Zarca, 22 $2^{\circ} 05^{\prime} 34^{\prime \prime} \mathrm{N}, 102^{\circ} 33^{\prime} 08^{\prime \prime} \mathrm{W}$, O. Rosales 2086 (HUAA); Barranca La Pinsión, Sierra de San Blas de Pabe-

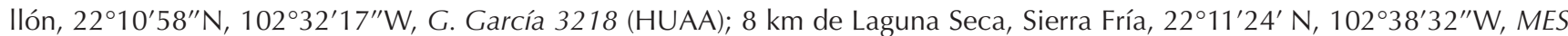
2277 (HUAA). Physalis cinerascens var. cinerascens. Mpio. Aguascalientes: El Sabinal, salto de Los Salado, $21^{\circ} 45^{\prime} 01^{\prime \prime} \mathrm{N}$,

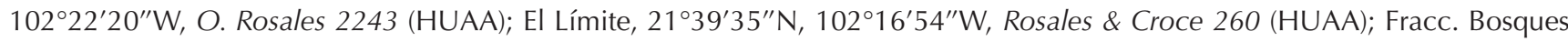

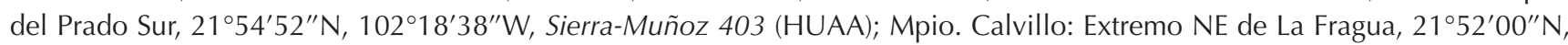

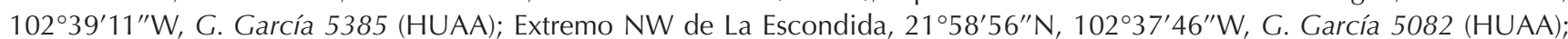
Mpio. Pabellón de Arteaga: $1.5 \mathrm{Km}$ del entronque de Pabellón de Arteaga-Rincón de Romos, $22^{\circ} 11^{\prime} 26^{\prime \prime} \mathrm{N}, 102^{\circ} 18^{\prime} 05^{\prime \prime} \mathrm{W}$, De la

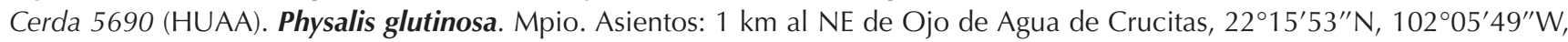


Solanaceae en Aguascalientes, México

Apéndice I. Continuación.

G. García 2946 (HUAA); Mpio. Calvillo: Cerro Blanco, O. Rosales 1869 (HUAA); Rancho El Carrizo, 2 Km al NE del Garruño,

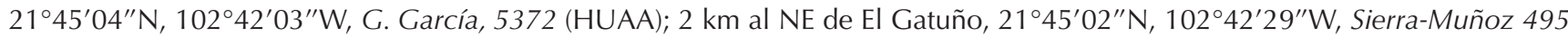
(HUAA); Presa Los Alamitos, 21 ${ }^{\circ} 43^{\prime} 46^{\prime \prime} \mathrm{N}, 102^{\circ} 42^{\prime} 44^{\prime \prime} \mathrm{W}$, Sierra-Muñoz 501 (HUAA); Barranca Montoro, $7.5 \mathrm{Km}$ al NW de Milpillas, $21^{\circ} 59^{\prime} 44^{\prime \prime} \mathrm{N}, 102^{\circ} 35^{\prime} 31^{\prime \prime} \mathrm{W}$, Sierra-Muñoz 636 (HUAA); $2.3 \mathrm{~km}$ al NE de la presa Los Alamitos, $21^{\circ} 44^{\prime} 40^{\prime \prime} \mathrm{N}, 102^{\circ} 41^{\prime} 09^{\prime \prime} \mathrm{W}$, M. E. Mendoza 797 (HUAA); Mpio. Cosío: Tanque La Punta, lado E del poblado La Punta, 22 $2^{\circ} 9^{\prime} 16^{\prime \prime} \mathrm{N}, 102^{\circ} 17^{\prime} 09^{\prime \prime} \mathrm{W}, \mathrm{O}^{\circ}$. Rosales 2044 (HUAA); Mpio. El Llano: Barranca La Palma, $5.35 \mathrm{~km}$ al NE de Palo Alto, $21^{\circ} 56^{\prime} 46^{\prime \prime} \mathrm{N}, 101^{\circ} 54^{\prime} 54^{\prime \prime} \mathrm{W}, \mathrm{M}^{\prime}$. E. Mendoza 597 (HUAA); Mpio. Rincón de Romos: Extremo NW de la Estancia de Mosqueira, 2208’42”N, 102²1'28”W, Sierra-Muñoz 421 (HUAA); Mpio. San José de Gracia: 3 km al E de La Congoja, sobre el camino a San José de Gracia, Rzedowski \& McVaugh 898 (MEXU); Barranca de Juan Francisco, Sierra San Blas de Pabellón, 2211'32”N, 102²1’05”W, G. García 2322 (HUAA; IEB); Camino al cerro de La Ardilla, cuesta La Gloria, 22 ${ }^{\circ} 10^{\prime} 56^{\prime \prime} \mathrm{N}, 102^{\circ} 30^{\prime} 24^{\prime \prime}$ W, De la Cerda-G. García 730 (HUAA); 11.75 km al NW de Rincón de Romos, $22^{\circ} 08^{\prime} 49^{\prime \prime} \mathrm{N}, 102^{\circ} 21^{\prime} 52^{\prime \prime} \mathrm{W}$, Sierra-Muñoz 558 (HUAA); 2.8 km al NW de la cortina de la presa Túnel de Potrerillo, 22 $14^{\prime} 52^{\prime \prime} \mathrm{N}, 102^{\circ} 27^{\prime} 40^{\prime \prime} \mathrm{W}$, J. Martínez 2245 (HUAA); Rancho Sierra Hermosa, 6 km al NW de La Congoja, $22^{\circ} 10^{\prime} 44^{\prime \prime} \mathrm{N}, 102^{\circ} 36^{\prime} 40^{\prime \prime} \mathrm{W}$, Sierra-Muñoz 694 (HUAA); Presa La Araña, 22 ${ }^{\circ} 13^{\prime} 40^{\prime \prime} \mathrm{N}, 102^{\circ} 37^{\prime} 21^{\prime \prime} \mathrm{W}$, Sierra-Muñoz 711 (HUAA);

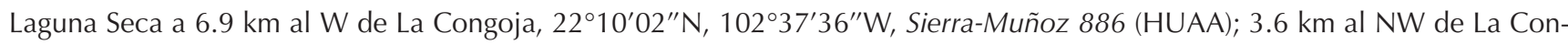
goja, $22^{\circ} 11^{\prime} 41^{\prime \prime} \mathrm{N}, 102^{\circ} 34^{\prime} 32^{\prime \prime} \mathrm{W}$, Sierra-Muñoz 894 (HUAA); Rancho Antrialgo, Barranca Juan Francisco a $4.6 \mathrm{~km}$ al NW de La

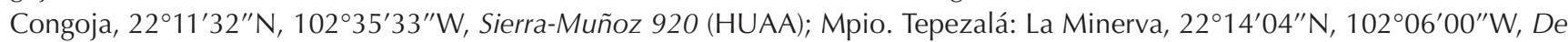
la Cerda-G. García 1427a (HUAA). Physalis hastatula. Mpio. El Llano: 1 km al SW de Palo Alto, Sierra-Muñoz 754 (HUAA); Barranca de La Mina, 6.7 km al NE de Palo Alto, M. E. Mendoza 613 (HUAA); Barranca La Palma, 5.35 km al NE de Palo Alto, M. E. Mendoza 596 (HUAA). Physalis lagascae. Mpio. Calvillo: Cruce de camino Río Gil-El Sauz, Sierra-Muñoz 439 (HUAA); Cruce de camino Río Gil-El Sauz, Sierra-Muñoz 441 (HUAA). Physalis nicandroides. Mpio. Aguascalientes: $1.5 \mathrm{~km}$ al NE de la CFE, carretera a San Bartolo, 21 ${ }^{\circ} 45^{\prime} 38^{\prime \prime} \mathrm{N}, 102^{\circ} 11^{\prime} 51^{\prime \prime} \mathrm{W}$, Sierra-Muñoz 465 (HUAA); Cerro El Picacho, 7 km al poniente de Ags., $21^{\circ} 53^{\prime} 34^{\prime \prime} \mathrm{N}, 102^{\circ} 24^{\prime} 46^{\prime \prime} \mathrm{W}, \mathrm{K}$. Landín 98 (HUAA); $9 \mathrm{~km}$ al SE del cruce carretera 45 a San Bartolo, 21 ${ }^{\circ} 45^{\prime} 04^{\prime \prime} \mathrm{N}, 102^{\circ} 12^{\prime} 47^{\prime \prime} \mathrm{W}$, Sierra-Muñoz 477 (HUAA); Granja Duarte, carr. Ags.-Calvillo, 2152'54”N, 102²1'49”'W, De la Cerda 4797 (HUAA); 1.6 km al $\mathrm{N}$ de la Hacienda San Bartolo, 21 ${ }^{\circ} 45^{\prime} 13^{\prime \prime} \mathrm{N}, 102^{\circ} 11^{\prime} 08^{\prime \prime} \mathrm{W}$, Sierra-Muñoz 813 (HUAA); $6.5 \mathrm{~km}$ al SW de Tanque de Los Jiménez, $21^{\circ} 39^{\prime} 41^{\prime \prime} \mathrm{N}, 102^{\circ} 24^{\prime} 49^{\prime \prime} \mathrm{W}$, Sierra-Muñoz 878 (HUAA); $4.27 \mathrm{~km}$ al SE de Villa de Lic. Jesús Terán, $21^{\circ} 47^{\prime} 08^{\prime \prime} \mathrm{N}, 102^{\circ} 12^{\prime} 06^{\prime \prime} \mathrm{W}$,

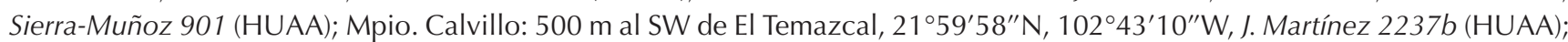

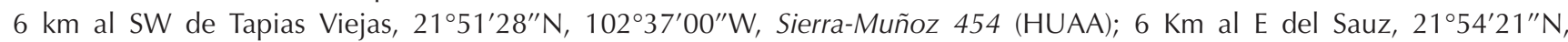

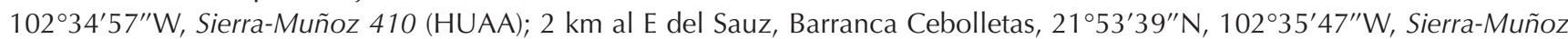

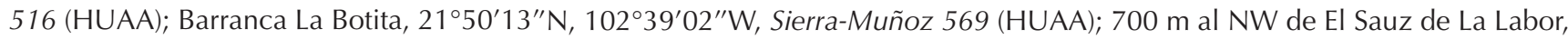
$22^{\circ} 00^{\prime} 06^{\prime \prime} \mathrm{N}, 102^{\circ} 39^{\prime} 07^{\prime \prime} \mathrm{W}$, Sierra-Muñoz 833 (HUAA); Barranca Cebolletas, $2.1 \mathrm{~km}$ al NE de El Sauz, 21 ${ }^{\circ} 53^{\prime} 17^{\prime \prime} \mathrm{N}, 102^{\circ} 35^{\prime} 47^{\prime \prime} \mathrm{W}$,

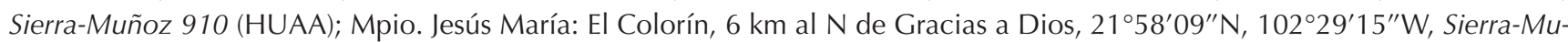

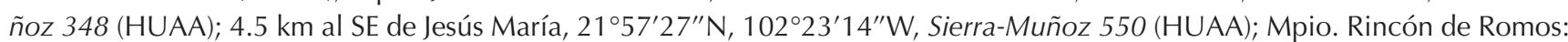
Barranca sierpes al E de Las Camas, $22^{\circ} 14^{\prime} 38^{\prime \prime} \mathrm{N}, 102^{\circ} 26^{\prime} 39^{\prime \prime} \mathrm{W}$, O. Rosales 1193 (HUAA); Extremo NW de la Estancia de Mos-

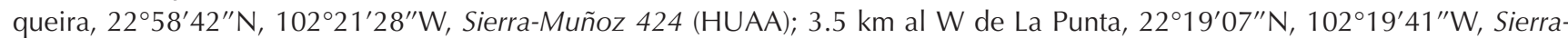
Muñoz 852 (HUAA); Mpio. San José de Gracia: Barranca de Revienta cuartas, Rancho Sierra Hermosa, 22 $2^{\circ} 10^{\prime} 21^{\prime \prime} \mathrm{N}, 102^{\circ} 36^{\prime} 43^{\prime \prime} \mathrm{W}$, G. Murillo 198 (HUAA). Physalis orizabae. Mpio. Aguascalientes: Ladera N del cerro El Picacho, 21 ${ }^{\circ} 53^{\prime} 13^{\prime \prime} \mathrm{N}, 102^{\circ} 25^{\prime} 06^{\prime \prime} \mathrm{W}, \mathrm{De}^{\prime}$ la Cerda-G. García 1026 (HUAA); Rumbo al cerro de Los Gallos, 2139'55”N, 102¹4’42”W, O. Rosales 2015 (HUAA); Ladera

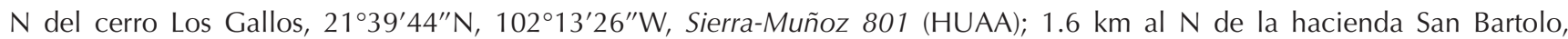
$21^{\circ} 45^{\prime} 13^{\prime \prime} \mathrm{N}, 102^{\circ} 11^{\prime} 08^{\prime \prime} \mathrm{W}$, Sierra-Muñoz 816 (HUAA); Mpio. Asientos: $4 \mathrm{~km}$ al SSE de Pilotos, $22^{\circ} 00^{\prime} 21^{\prime \prime} \mathrm{N}, 101^{\circ} 56^{\prime} 26^{\prime \prime} \mathrm{W}$, Sierra-Muñoz 778 (HUAA); Mpio. Calvillo: Sierra el Laurel, near the Jalisco-Aguascalientes border, ca. 10 miles southeast of Calvilo, McVaugh 18439 (ENCB); El Guarache, $15 \mathrm{~km}$ al N de El Terrero del Refugio, 2204'27”N, 102³9'20”W, G. García 4864

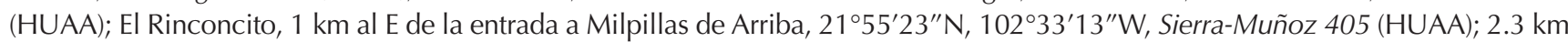

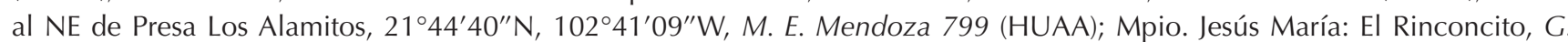

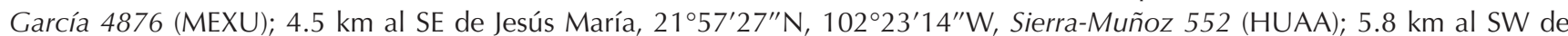
Tapias Viejas, $21^{\circ} 49^{\prime} 00^{\prime \prime} \mathrm{N}, 102^{\circ} 35^{\prime} 05^{\prime \prime} \mathrm{W}$, G. Murillo 338 (HUAA); $3.2 \mathrm{~km}$ al NW de Gracias a Dios, 21 ${ }^{\circ} 56^{\prime} 50^{\prime \prime} \mathrm{N}, 102^{\circ} 30^{\prime} 59^{\prime \prime} \mathrm{W}$, M. E. Mendoza 693 (HUAA); Mpio. San Francisco de los Romo: 4.7 km al W de Hacienda El Garabato, 22 $2^{\circ} 04^{\prime} 57^{\prime \prime} \mathrm{N}, 102^{\circ} 23^{\prime} 10^{\prime \prime} \mathrm{W}$, G. Murillo 453 (HUAA); Mpio. San José de Gracia: 200 m delante de Laguna Seca, parque natural Sierra Fría, O. Vargas 776

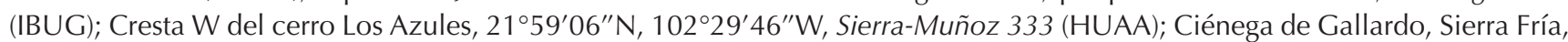
$22^{\circ} 08^{\prime} 16^{\prime \prime} \mathrm{N}, 102^{\circ} 39^{\prime} 87^{\prime \prime} \mathrm{W}$, Adame-Clark-Alfonso 744 (HUAA); Barranca La Tórtola, $1 \mathrm{~km}$ al NW de Playa Mariquitas, $22^{\circ} 16^{\prime} 04^{\prime \prime} \mathrm{N}, 102^{\circ} 37^{\prime} 21^{\prime \prime} \mathrm{W}$, Sierra-Muñoz 354 (HUAA); Rancho Sierra Hermosa, $6 \mathrm{~km}$ al NW de La Congoja, 22 ${ }^{\circ} 10^{\prime} 44^{\prime \prime} \mathrm{N}$,

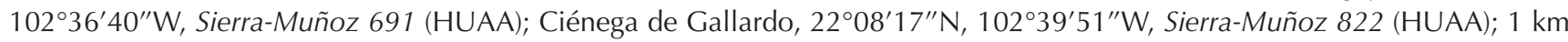


José CARlos Sierra-MuÑoz ET AL.

Apéndice I. Continuación.

al E de Ciénega de Gallardo, 22 $2^{\circ} 08^{\prime} 06^{\prime \prime} \mathrm{N}, 102^{\circ} 34^{\prime} 24^{\prime \prime} \mathrm{W}$, Sierra-Muñoz 824 (HUAA); 2 km al W de Ciénega de Gallardo, $22^{\circ} 08^{\prime} 19^{\prime \prime} \mathrm{N}, 102^{\circ} 40^{\prime} 55^{\prime \prime} \mathrm{W}$, Sierra-Muñoz 829 (HUAA). Physalis patula. Mpio. Aguascalientes: 3 km al NE de Los Caños, $21^{\circ} 48^{\prime} 37^{\prime \prime} \mathrm{N}, 102^{\circ} 27^{\prime} 02^{\prime \prime} \mathrm{W}$, Sierra-Muñoz 604 (HUAA); Granja Duarte, carr. Ags.-Calvillo, De la Cerda 4797 (IEB); Mpio. Asientos: $1.76 \mathrm{~km}$ al SE de El Tule, $22^{\circ} 01^{\prime} 58^{\prime \prime} \mathrm{N}, 102^{\circ} 05^{\prime} 51^{\prime \prime} \mathrm{W}, \mathrm{M}$. E. Mendoza 763 (HUAA); Mpio. Calvillo: $2 \mathrm{~km}$ al SE de presa de Los Serna, $21^{\circ} 48^{\prime} 02^{\prime \prime} \mathrm{N}, 102^{\circ} 49^{\prime} 13^{\prime \prime} \mathrm{W}$, G. Murillo 473 (HUAA); Mpio. El Llano: Barranca de La Mina, $6.7 \mathrm{~km}$ al NE de Palo Alto,

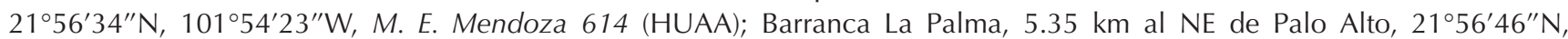

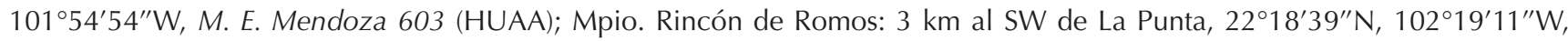
Sierra-Muñoz 842 (HUAA); Mpio. San Francisco de los Romo: 2 km al E de Borrotes, 22 $02^{\prime} 04^{\prime \prime} \mathrm{N}, 102^{\circ} 11^{\prime} 14^{\prime \prime} \mathrm{W}$, Sierra-Muñoz

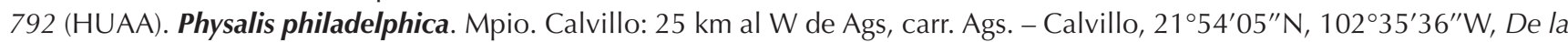

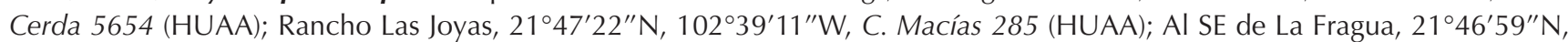

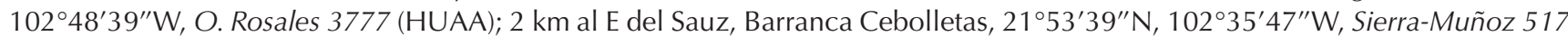

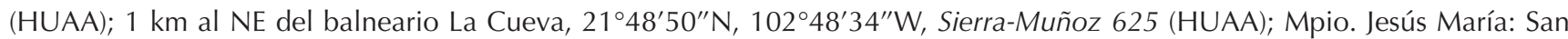

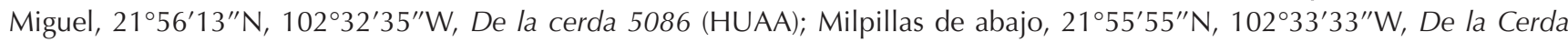

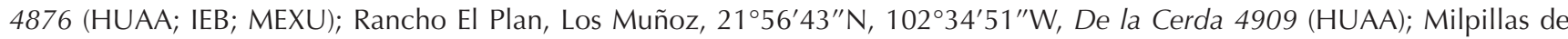
Abajo, De la Cerda 4880 (IEB). Physalis pruinosa. Mpio. Calvillo: $500 \mathrm{~m}$ al S de presa de Los Serna, $21^{\circ} 47^{\prime} 56^{\prime \prime} \mathrm{N}, 102^{\circ} 50^{\prime} 25^{\prime \prime} \mathrm{W}$,

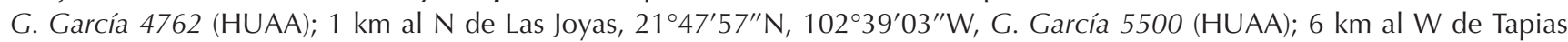

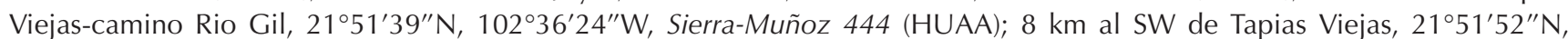
$102^{\circ} 37^{\prime} 16^{\prime \prime} \mathrm{W}$, Sierra-Muñoz 461 (HUAA); $700 \mathrm{~m}$ al NW de El Sauz de La Labor, 22 ${ }^{\circ} 00^{\prime} 06^{\prime \prime} \mathrm{N}, 102^{\circ} 39^{\prime} 07^{\prime \prime} \mathrm{W}$, Sierra-Muñoz 834

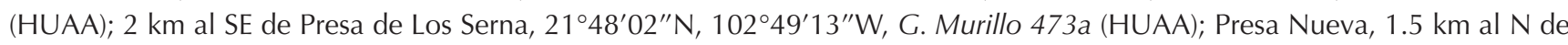
Presa de Los Serna, $21^{\circ} 49^{\prime} 16^{\prime \prime} \mathrm{N}, 102^{\circ} 50^{\prime} 21^{\prime \prime} \mathrm{W}$, G. Murillo 476 (HUAA). Physalis pubescens. Mpio. Aguascalientes: 9 km al SSE

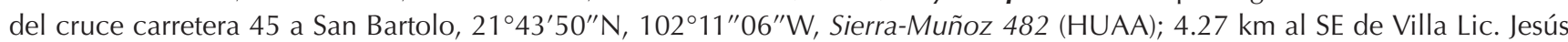

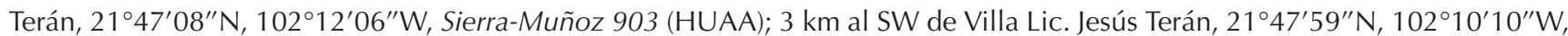
Sierra-Muñoz 905 (HUAA); Mpio. Calvillo: El Pilar, $10 \mathrm{~km}$ al N de El Temazcal, 2203'52”N, 10243'09”W, G. García 3517 (HUAA); 7 km al SE de Jaltiche de Arriba, $21^{\circ} 44^{\prime} 31^{\prime \prime} \mathrm{N}, 102^{\circ} 45^{\prime} 00^{\prime \prime} \mathrm{W}$, Sierra-Muñoz 485 (HUAA). Physalis solanaceus. Mpio. Aguascalientes: $8.1 \mathrm{~km}$ al E de Ags. $1 \mathrm{~km}$ al NE de Calvillito, 21 ${ }^{\circ} 49^{\prime} 56^{\prime \prime} \mathrm{N}, 102^{\circ} 12^{\prime} 04^{\prime \prime} \mathrm{W}$, De la Cerda 5895 (HUAA); $6 \mathrm{~km}$ antes del cerro de Los Gallos, $21^{\circ} 39^{\prime} 47^{\prime \prime} \mathrm{N}, 102^{\circ} 13^{\prime} 47^{\prime \prime} \mathrm{W}$, De la Cerda 1685 (HUAA); $1 \mathrm{~km}$ al S del rancho El Catorce carr. Ags.-Villa

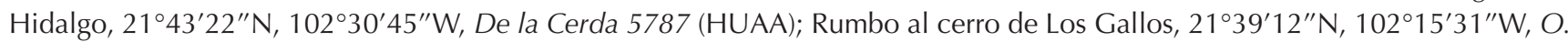

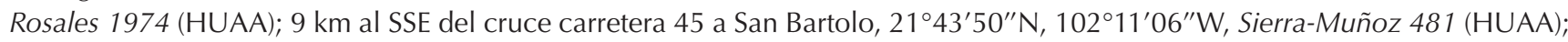

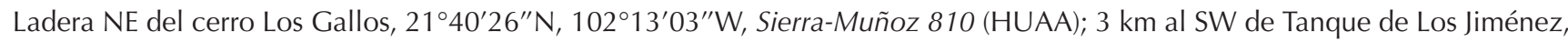
$21^{\circ} 41^{\prime} 01^{\prime \prime} \mathrm{N}, 102^{\circ} 23^{\prime} 22^{\prime \prime} \mathrm{W}$, Sierra-Muñoz 877 (HUAA); $6.5 \mathrm{~km}$ al SW de Tanque de Los Jiménez, $21^{\circ} 39^{\prime} 41^{\prime \prime} \mathrm{N}, 102^{\circ} 24^{\prime} 49^{\prime \prime} \mathrm{W}$,

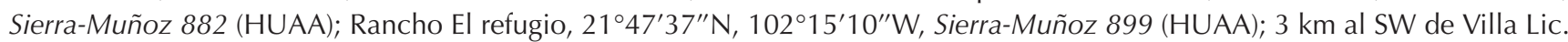

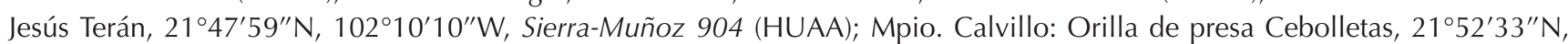

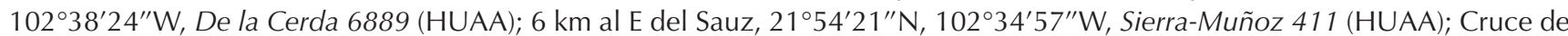

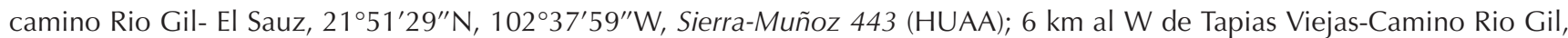
$21^{\circ} 51^{\prime} 39^{\prime \prime} \mathrm{N}, 102^{\circ} 36^{\prime} 24^{\prime \prime} \mathrm{W}$, Sierra-Muñoz 452 (HUAA); $9 \mathrm{~km}$ al SSE del cruce carretera 45 a San Bartolo, 21 $43^{\prime} 50^{\prime \prime} \mathrm{N}$, $102^{\circ} 11^{\prime} 06^{\prime \prime} \mathrm{W}$, Sierra-Muñoz 481 (HUAA); $7 \mathrm{~km}$ al SE de Jaltiche de Arriba, $21^{\circ} 44^{\prime} 31^{\prime \prime} \mathrm{N}, 102^{\circ} 45^{\prime} 00^{\prime \prime} \mathrm{W}$, Sierra-Muñoz 484

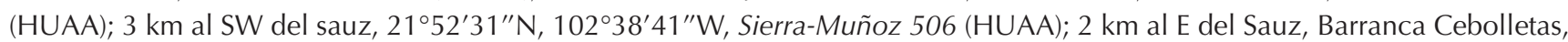

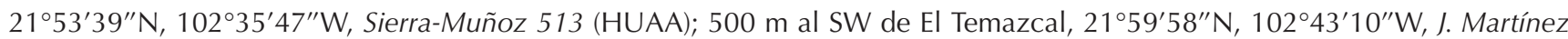
2230 (HUAA); $950 \mathrm{~m}$ al NE del Terrero de la Labor, $22^{\circ} 01^{\prime} 50^{\prime \prime} \mathrm{N}, 102^{\circ} 39^{\prime} 50^{\prime \prime} \mathrm{W}$, I. Ortiz 53 (HUAA); Mpio. Jesús María: El Colo-

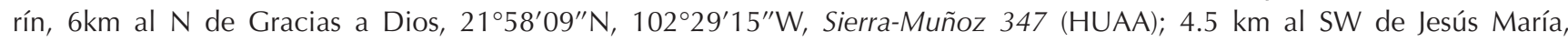
$21^{\circ} 57^{\prime} 47^{\prime \prime} \mathrm{N}, 102^{\circ} 23^{\prime} 18^{\prime \prime} \mathrm{W}$, Sierra-Muñoz 544 (HUAA); Mpio. Pabellón de Arteaga: Ladera W del cerro el Chiquihuitillo, 1 km al NW de la ex hacienda del mezquite, $22^{\circ} 05^{\prime} 15^{\prime \prime} \mathrm{N}, 102^{\circ} 12^{\prime} 17^{\prime \prime} \mathrm{W}$, Sierra-Muñoz 365 (HUAA). Physalis virginiana. Mpio. Pabellón de Arteaga: Ladera W del cerro El Chiquihuitillo, $1 \mathrm{~km}$ al NW de la ex hacienda de EL Mezquite, $22^{\circ} 05^{\prime} 15^{\prime \prime} \mathrm{N}, 102^{\circ} 12^{\prime} 17^{\prime \prime} \mathrm{W}$, Sierra-Muñoz 366 (HUAA); Mpio. Tepezalá: Arroyo Las Pilas, 2 km al W de la ex hacienda Las Pilas, 22 ${ }^{\circ} 15^{\prime} 59^{\prime \prime} \mathrm{N}, 102^{\circ} 10^{\prime} 50^{\prime \prime} \mathrm{W}$,

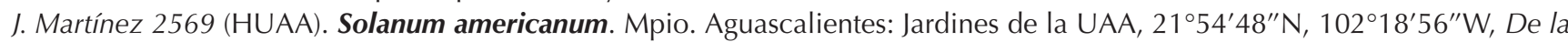
Cerda 4535a (HUAA); Barranca a $5 \mathrm{~km}$ al E de Malpaso, 21 ${ }^{\circ} 49^{\prime} 55^{\prime \prime} \mathrm{N}, 102^{\circ} 38^{\prime} 48^{\prime \prime} \mathrm{W}$, De la Cerda 4549 (HUAA); Presa de San

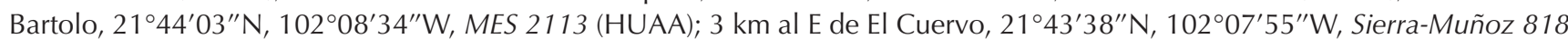

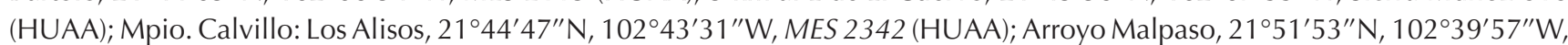
G. Nieto 106 (HUAA); 1 km al SE de Malpaso, Glez.-Adame 280 (HUAA); Barranca Las Moras, $1 \mathrm{~km}$ al SE de la cortina de la

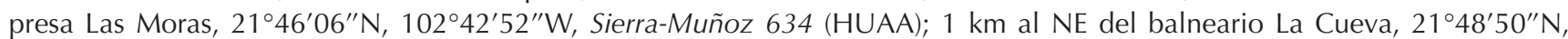
$102^{\circ} 48^{\prime} 34^{\prime \prime} \mathrm{W}$, Sierra-Muñoz 623 (HUAA); $250 \mathrm{~m}$ al W del balneario La Cueva, $21^{\circ} 48^{\prime} 37^{\prime \prime} \mathrm{N}, 102^{\circ} 49^{\prime} 07^{\prime \prime} \mathrm{W}$, Sierra-Muñoz 619 
Solanaceae en Aguascalientes, México

Apéndice I. Continuación.

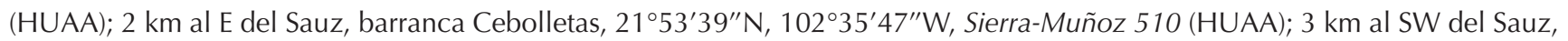

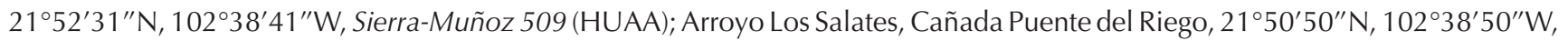

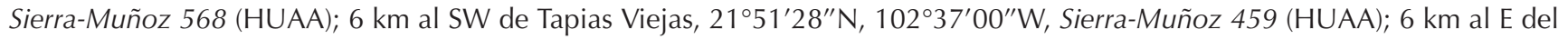

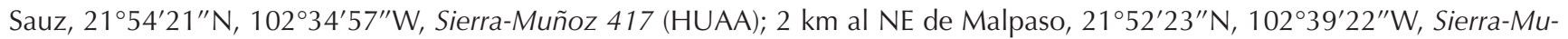

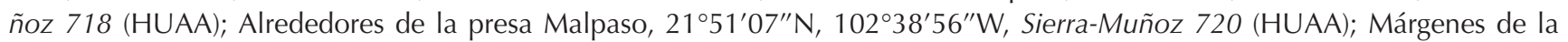

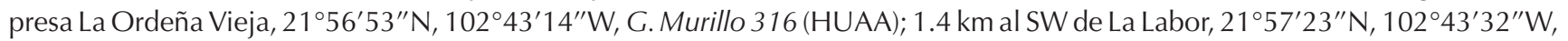
G. García 6139 (HUAA); Mpio. Cosío: 1.8 km al NW de Cosío, 22²2’15” N, 102¹8’58”W, Sierra-Muñoz 663 (HUAA); Mpio.

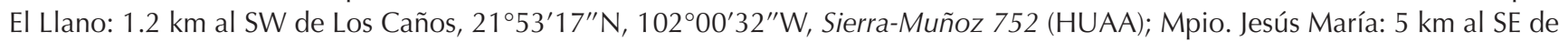

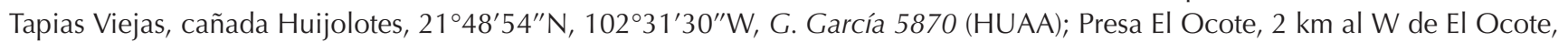

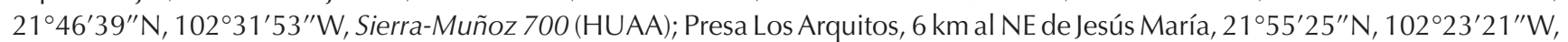

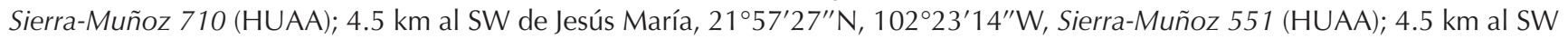
de Jesús María, 1.5 km de la Posta Zootécnica, 21 $57^{\prime} 49^{\prime \prime} \mathrm{N}, 102^{\circ} 23^{\prime} 18^{\prime \prime} \mathrm{W}$, Sierra-Muñoz 545 (HUAA); Mpio. Pabellón de Arteaga: Arroyo Santiago, 2 km al W de Santiago, $22^{\circ} 07^{\prime} 17^{\prime \prime} \mathrm{N}, 102^{\circ} 21^{\prime} 19^{\prime \prime} \mathrm{W}$, M. E. Mendoza 587 (HUAA); Mpio. Rincón de Romos: $3.5 \mathrm{~km}$ al W de La Punta, $22^{\circ} 19^{\prime} 07^{\prime \prime} \mathrm{N}, 102^{\circ} 19^{\prime} 41^{\prime \prime} \mathrm{W}$, Sierra-Muñoz 856 (HUAA); Arroyo La Boquilla, $800 \mathrm{~m}$ al W de La BoquiIla, $22^{\circ} 15^{\prime} 50^{\prime \prime} \mathrm{N}, 102^{\circ} 22^{\prime} 37^{\prime \prime} \mathrm{W}, \mathrm{M}$. E. Mendoza 578 (HUAA); Mpio. San José de Gracia: Río Blanco, 22 $09^{\prime} 24^{\prime \prime} \mathrm{N}, 102^{\circ} 28^{\prime} 52^{\prime \prime} \mathrm{W}$, MES 3166 (HUAA); $700 \mathrm{~m}$ al W de la cortina de la presa Calles, $22^{\circ} 08^{\prime} 17^{\prime \prime} \mathrm{N}, 102^{\circ} 24^{\prime} 47^{\prime \prime} \mathrm{W}, \mathrm{M}$. E. Mendoza 427 (HUAA); $700 \mathrm{~m}$ de la cortina de la presa Calles, $22^{\circ} 08^{\prime} 17^{\prime \prime} \mathrm{N}, 102^{\circ} 24^{\prime} 47^{\prime \prime} \mathrm{W}$, F. Cabrera 336 (HUAA); $3 \mathrm{~km}$ al NW de la cortina de la presa El Jocoqui, $22^{\circ} 08^{\prime} 04^{\prime \prime} \mathrm{N}, 102^{\circ} 22^{\prime} 55^{\prime \prime} \mathrm{W}$, Sierra-Muñoz 559 (HUAA); Boca de Túnel de Potrerillo, 22 $2^{\circ} 14^{\prime} 34^{\prime \prime} \mathrm{N}, 102^{\circ} 26^{\prime} 43^{\prime \prime} \mathrm{W}$, Sie- $^{\circ}$

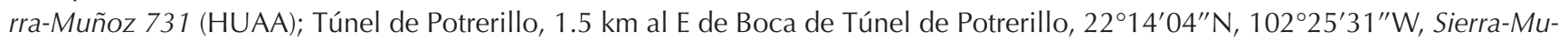
ñoz 734 (HUAA); Cortina de la presa 50 Aniversario, $22^{\circ} 11^{\prime} 04^{\prime \prime} \mathrm{N}, 102^{\circ} 27^{\prime} 53^{\prime \prime} \mathrm{W}$, Sierra-Muñoz 743 (HUAA); Margen NW de la presa Calles, $22^{\circ} 09^{\prime} 42^{\prime \prime} \mathrm{N}, 102^{\circ} 27^{\prime} 22^{\prime \prime} \mathrm{W}$, M. E. Mendoza 544 (HUAA); Mpio. Tepezalá: Presa Mesillas, 1 km al SE de Mesillas, $22^{\circ} 18^{\prime} 43^{\prime \prime} \mathrm{N}, 102^{\circ} 09^{\prime} 40^{\prime \prime} \mathrm{W}$, Sierra-Muñoz 670 (HUAA). Solanum cardiophyllum. Mpio. Aguascalientes: $1.5 \mathrm{~km}$ al NE de la CFE, carretera a San Bartolo, Sierra-Muñoz 466 (HUAA); 1.6 km al N de Hacienda San Bartolo, Sierra-Muñoz 815 (HUAA). Solanum corymbosum. Mpio. Tepezalá: 3 km al SW de Tepezalá, G. García 4080 (HUAA); 2.7 km al SE de Tepezalá, Sierra-Muñoz 678 (HUAA). Solanum dasyadenium. Mpio. Aguascalientes: Ladera N del cerro Los Gallos, $21^{\circ} 39^{\prime} 44^{\prime \prime} \mathrm{N}, 1^{\circ} 2^{\circ} 13^{\prime} 26^{\prime \prime} \mathrm{W}$, Sierra-Mu- $^{\prime}$ ñoz 805 (HUAA); Rancho El Refugio, 21 ${ }^{\circ} 47^{\prime} 37^{\prime \prime} \mathrm{N}, 102^{\circ} 15^{\prime} 10^{\prime \prime} \mathrm{W}$, Sierra-Muñoz 897 (HUAA); Mpio. Calvillo: Cañada del Temazcal, $0.5 \mathrm{~km}$ al S de presa El Temazcal, 21 $58^{\prime} 54^{\prime \prime} \mathrm{N}, 102^{\circ} 43^{\prime} 13^{\prime \prime} \mathrm{W}$, J. Martínez 1683 (HUAA); Barranca Montoro, $7.5 \mathrm{~km}$ al NW de Milpillas, $21^{\circ} 59^{\prime} 44^{\prime \prime} \mathrm{N}, 102^{\circ} 35^{\prime} 31^{\prime \prime} \mathrm{W}$, Sierra-Muñoz 637 (HUAA); Barranca Las Moras, $1 \mathrm{~km}$ al SE de la cortina de la presa

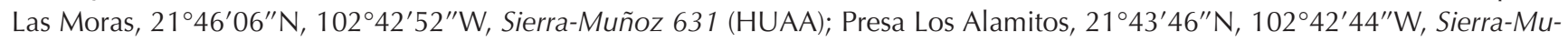

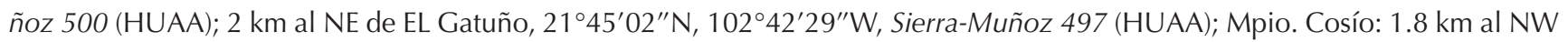
de Cosío, 22 $2^{\circ} 22^{\prime} 15^{\prime \prime} \mathrm{N}, 102^{\circ} 18^{\prime} 58^{\prime \prime} \mathrm{W}$, Sierra-Muñoz 662 (HUAA); Mpio. Pabellón de Arteaga: Arroyo Santiago, 2 km al W de Santiago, $22^{\circ} 07^{\prime} 17^{\prime \prime} \mathrm{N}, 102^{\circ} 21^{\prime} 19^{\prime \prime} \mathrm{W}$, M. E. Mendoza 586 (HUAA); Mpio. San José de Gracia: Presa Calles, $22^{\circ} 09^{\prime} 00^{\prime \prime} \mathrm{N}$, $102^{\circ} 25^{\prime} 18^{\prime \prime} \mathrm{W}$, MES 2304 (HUAA); $700 \mathrm{~m}$ al E de la cortina de la presa Calles, $22^{\circ} 08^{\prime} 17^{\prime \prime} \mathrm{N}, 102^{\circ} 24^{\prime} 47^{\prime \prime} \mathrm{W}, \mathrm{G}^{\circ}$ García 5941 (HUAA); $700 \mathrm{~m}$ al W de la estación Biológica Agua Zarca, 22 ${ }^{\circ} 05^{\prime} 03^{\prime \prime} \mathrm{N}, 102^{\circ} 33^{\prime} 13^{\prime \prime} \mathrm{W}$, Sierra-Muñoz 531 (HUAA); Boca de Túnel de Potrerillo, 22 ${ }^{\circ} 14^{\prime} 34^{\prime \prime} \mathrm{N}, 102^{\circ} 26^{\prime} 43^{\prime \prime} \mathrm{W}$, Sierra-Muñoz 728 (HUAA); Túnel de Potrerillo, $1.5 \mathrm{~km}$ al E de Boca de Túnel de Potrerillo, $22^{\circ} 14^{\prime} 04^{\prime \prime} \mathrm{N}, 102^{\circ} 25^{\prime} 31^{\prime \prime} \mathrm{W}$, Sierra-Muñoz 733 (HUAA); Rancho Antrialgo, Barranca Juan Francisco a $4.6 \mathrm{~km}$ al NW de La Congoja, $22^{\circ} 11^{\prime} 32^{\prime \prime} \mathrm{N}, 102^{\circ} 35^{\prime} 33^{\prime \prime}$ W, Sierra-Muñoz 919 (HUAA); Mpio. Tepezalá: Presa Mesillas, 1 km al SE de Mesillas, $22^{\circ} 18^{\prime} 43^{\prime \prime} \mathrm{N}, 102^{\circ} 09^{\prime} 40^{\prime \prime} \mathrm{W}$, Sierra-Muñoz 667 (HUAA). Solanum deflexum. Mpio. Calvillo: Lomeríos a $3 \mathrm{~km}$ al SE de Presa de Los Serna, $21^{\circ} 47^{\prime} 54^{\prime \prime} \mathrm{N}, 102^{\circ} 49^{\prime} 04^{\prime \prime} \mathrm{W}$, J. Martínez 2056 (HUAA); $600 \mathrm{~m}$ al SSE de El Sauz de La Labor, 21 ${ }^{\circ} 59^{\prime} 35^{\prime \prime} \mathrm{N}, 102^{\circ} 38^{\prime} 52^{\prime \prime} \mathrm{W}$,

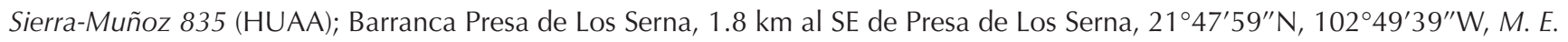
Mendoza 618 (HUAA). Solanum demissum. Mpio. San José de Gracia: Playa Cebolletas, Monte Grande de Sierra fría, $22^{\circ} 07^{\prime} 32^{\prime \prime} \mathrm{N}$, 102 38'10"W, G. García 3209 (HUAA; IEB); 1 km al W de Puerto del Aire, 22 ${ }^{\circ} 16^{\prime} 50^{\prime \prime} \mathrm{N}, 102^{\circ} 36^{\prime} 38^{\prime \prime}$ W, Sierra-Muñoz 360 (HUAA). Solanum dulcamaroides. Mpio. Calvillo: 1 km al W del Sauz de la Labor, De la Cerda-G. García 849 (HUAA). Solanum

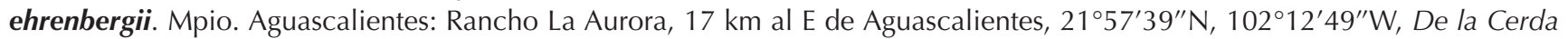

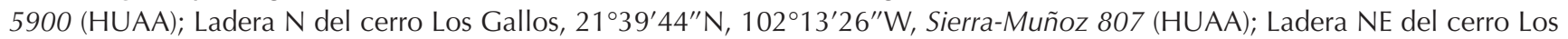

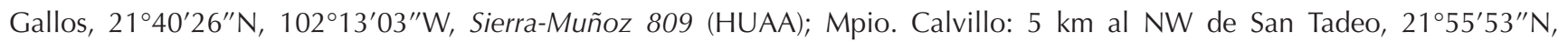

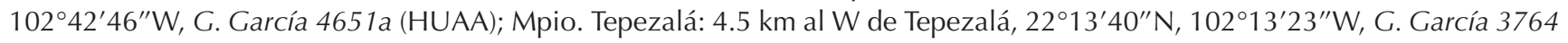
(HUAA). Solanum elaeagnifolium. Mpio. Aguascalientes: El Salto de Los Salado, $21^{\circ} 42^{\prime} 47^{\prime \prime} N, 1^{\circ} 22^{\circ} 34^{\prime \prime}$ W, M. Croce s.n.

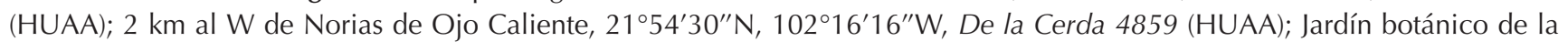

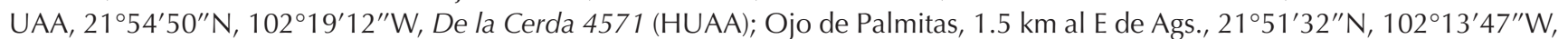

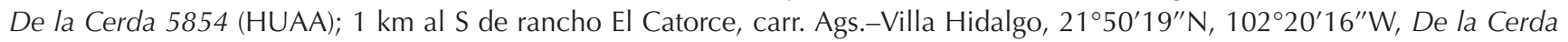


José CARlos Sierra-MuÑoz ET AL.

Apéndice I. Continuación.

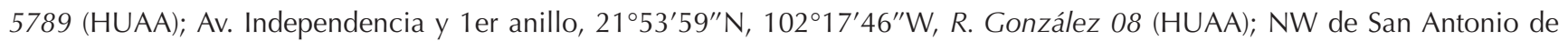
Peñuelas, $21^{\circ} 40^{\prime} 50^{\prime \prime} \mathrm{N}, 102^{\circ} 18^{\prime} 34^{\prime \prime} \mathrm{W}$, De la Cerda 5341 (HUAA); $1.5 \mathrm{~km}$ al NE de la CFE, carretera a San Bartolo, $21^{\circ} 45^{\prime} 38^{\prime \prime} \mathrm{N}$, $102^{\circ} 11^{\prime} 51^{\prime \prime} \mathrm{W}$, Sierra-Muñoz 468 (HUAA); Alrededores de la presa El Niagara, 21 $46^{\prime} 49^{\prime \prime} \mathrm{N}, 102^{\circ} 21^{\prime} 56^{\prime \prime} \mathrm{W}$, Sierra-Muñoz 612

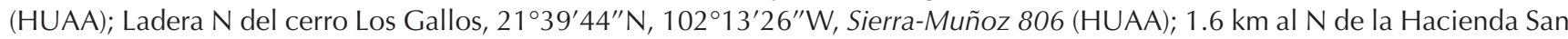

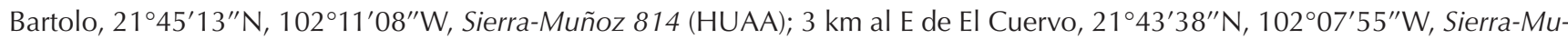

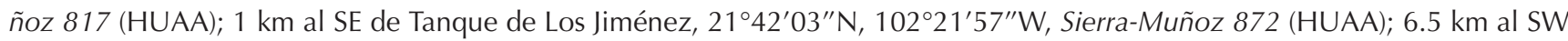
de Tanque de Los Jiménez, $21^{\circ} 39^{\prime} 41^{\prime \prime} \mathrm{N}, 102^{\circ} 24^{\prime} 49^{\prime \prime} \mathrm{W}$, Sierra-Muñoz 880 (HUAA); Mpio. Asientos: Presa Los Alcantares, Villa Juárez, $22^{\circ} 06^{\prime} 11^{\prime \prime} \mathrm{N}, 102^{\circ} 02^{\prime} 34^{\prime \prime} \mathrm{W}$, A. Lugo 14 (HUAA); Bordo al extremo SW de Plutarco Elías Calles, 22 $2^{\circ} 12^{\prime} 32^{\prime \prime} \mathrm{N}, 102^{\circ} 03^{\prime} 25^{\prime \prime} \mathrm{W}$, I. Ortiz 02 (HUAA); 6 km de San Gil. Valle del Chicalote, $22^{\circ} 09^{\prime} 59^{\prime \prime} \mathrm{N}, 102^{\circ} 04^{\prime} 23^{\prime \prime} \mathrm{W}, \mathrm{MES} 2223$ (HUAA); $500 \mathrm{~m}$ al E de Puerto de La Concepción, $22^{\circ} 12^{\prime} 10^{\prime \prime} \mathrm{N}, 102^{\circ} 07^{\prime} 52^{\prime \prime} \mathrm{W}$, Sierra-Muñoz 766 (HUAA); $2.25 \mathrm{~km}$ al SSE de Pilotos, 22 $01^{\prime} 02^{\prime \prime} \mathrm{N}, 1^{\circ} 01^{\circ} 56^{\prime} 53^{\prime \prime} \mathrm{W}$, Sierra-Muñoz 775 (HUAA); $1.4 \mathrm{~km}$ al NE de Los Molinos, 22 $2^{\circ} 09^{\prime} 51^{\prime \prime} \mathrm{N}, 102^{\circ} 04^{\prime} 41^{\prime \prime} \mathrm{W}$, M. E. Mendoza 776 (HUAA); $1 \mathrm{~km}$ al NW de Pino Suárez, $22^{\circ} 10^{\prime} 25^{\prime \prime} \mathrm{N}, 102^{\circ} 06^{\prime} 14^{\prime \prime} \mathrm{W}$, M. E. Mendoza 767 (HUAA); Mpio. Calvillo: El rinconcito, 1 km al E de la entrada

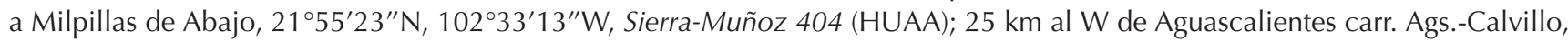

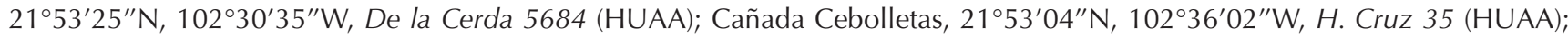

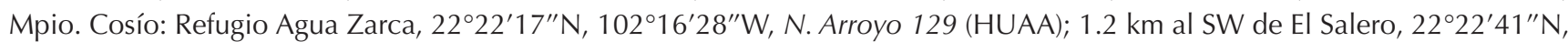
$102^{\circ} 20^{\prime} 03^{\prime \prime} \mathrm{W}$, M. E. Mendoza 704 (HUAA); Mpio. El Llano: $1.7 \mathrm{~km}$ al NE del Tule, 21 ${ }^{\circ} 54^{\prime} 45^{\prime \prime} \mathrm{N}, 102^{\circ} 55^{\prime} 06^{\prime \prime} \mathrm{W}, \mathrm{F}$. Cabrera 305 (HUAA); Rancho San Sebastián a $4.7 \mathrm{~km}$ al SE de Calvillito, 21 $50^{\circ} 10^{\prime \prime} \mathrm{N}, 102^{\circ} 08^{\prime} 30^{\prime \prime} \mathrm{W}$, Sierra-Muñoz 749 (HUAA); $1.2 \mathrm{~km}$ al SW de Los Caños, $21^{\circ} 53^{\prime} 17^{\prime \prime} \mathrm{N}, 102^{\circ} 00^{\prime} 32^{\prime \prime} \mathrm{W}$, Sierra-Muñoz 750 (HUAA); $3.5 \mathrm{~km}$ al NE de Palo Alto, 21 ${ }^{\circ} 56^{\prime} 40^{\prime \prime} \mathrm{N}, 101^{\circ} 56^{\prime} 45^{\prime \prime} \mathrm{W}$, Sierra-Muñoz 761 (HUAA); $1.5 \mathrm{~km}$ al NE de Ojo de Agua de Crucitas, 21 ${ }^{\circ} 58^{\prime} 04^{\prime \prime} \mathrm{N}, 101^{\circ} 56^{\prime} 12^{\prime \prime} \mathrm{W}$, Sierra-Muñoz 780 (HUAA);

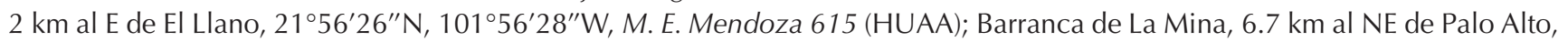

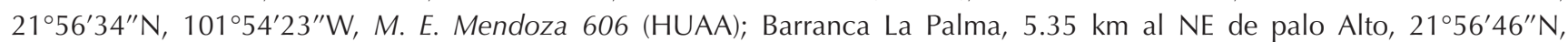

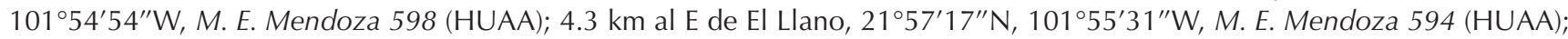

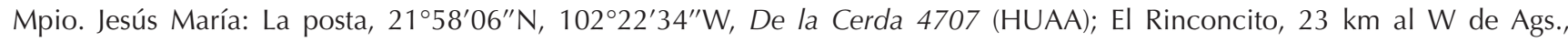
$21^{\circ} 55^{\prime} 25^{\prime \prime} \mathrm{N}, 102^{\circ} 32^{\prime} 47^{\prime \prime} \mathrm{W}$, De la Cerda 5647 (HUAA); San Isidro, carr. Calvillo - Tapias Viejas, $22^{\circ} 01^{\prime} 10^{\prime \prime} \mathrm{N}, 1^{\circ} 2^{\circ} 08^{\prime} 16^{\prime \prime} \mathrm{W}, \mathrm{De}^{\prime}$

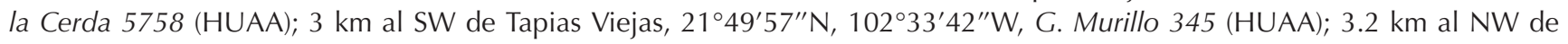

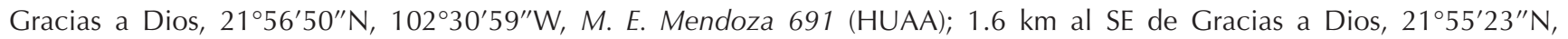
$102^{\circ} 28^{\prime} 36^{\prime \prime} \mathrm{W}$, M. E. Mendoza 690 (HUAA); $2.8 \mathrm{~km}$ al SE de Gracias a Dios, $21^{\circ} 54^{\prime} 51^{\prime \prime} \mathrm{N}, 102^{\circ} 28^{\prime} 06^{\prime \prime} \mathrm{W}, \mathrm{M}$. E. Mendoza 685

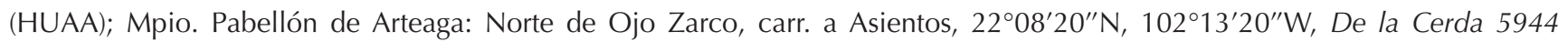

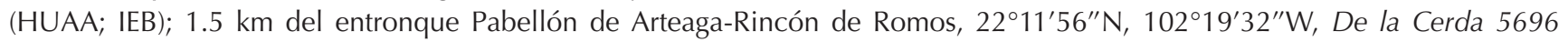
(HUAA; IEB); Arroyo Santiago, $2 \mathrm{~km}$ al W de Santiago, $22^{\circ} 07^{\prime} 17^{\prime \prime} \mathrm{N}, 102^{\circ} 21^{\prime} 19^{\prime \prime} \mathrm{W}, \mathrm{M}$. E. Mendoza 582 (HUAA); $1 \mathrm{~km}$ al W de Santiago, $22^{\circ} 07^{\prime} 00^{\prime \prime} \mathrm{N}, 102^{\circ} 20^{\prime} 52^{\prime \prime} \mathrm{W}$, M. E. Mendoza 580 (HUAA); Mpio. Rincón de Romos: 3 km al SW de La Punta, 22 ${ }^{\circ} 18^{\prime} 39^{\prime \prime} \mathrm{N}$,

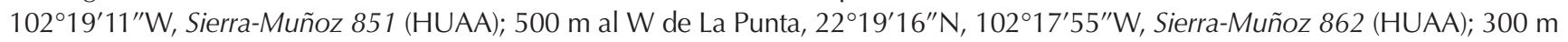
al N de Escaleras, $22^{\circ} 15^{\prime} 26^{\prime \prime} \mathrm{N}, 102^{\circ} 19^{\prime} 54^{\prime \prime} \mathrm{W}$, Sierra-Muñoz 863 (HUAA); Mpio. San Francisco de los Romo: 2 km al SE de San

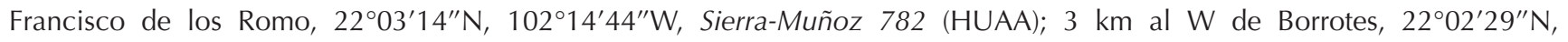
$102^{\circ} 13^{\prime} 58^{\prime \prime} \mathrm{W}$, Sierra-Muñoz 785 (HUAA); 2 km al W de Borrotes, 2202'12”N, 102¹3'29”W, Sierra-Muñoz 788 (HUAA); 1.5 km al NW de Borrotes, $22^{\circ} 02^{\prime} 34^{\prime \prime} \mathrm{N}, 102^{\circ} 13^{\prime} 04^{\prime \prime}$ W, Sierra-Muñoz 791 (HUAA); Mpio. San José de Gracia: 1 km al NW de San Antonio de los Ríos, $22^{\circ} 09^{\prime} 56^{\prime \prime} \mathrm{N}, 102^{\circ} 28^{\prime} 58^{\prime \prime} \mathrm{W}$, De la Cerda 5720 (HUAA; IEB); 3 km al N de San José de Gracia, carretera a La Congoja, $22^{\circ} 10^{\prime} 25^{\prime \prime} \mathrm{N}, 102^{\circ} 25^{\prime} 10^{\prime \prime} \mathrm{W}$, Sierra-Muñoz 724 (HUAA); Mpio. Tepezalá: $1 \mathrm{~km}$ al W de Tepezalá, 22 ${ }^{\circ} 13^{\prime} 31^{\prime \prime} \mathrm{N}$, $102^{\circ} 11^{\prime} 27^{\prime \prime} \mathrm{W}$, De la Cerda 4740 (HUAA); 2.7 km al SE de Tepezalá, 22 ${ }^{\circ} 15^{\prime} 50^{\prime \prime} \mathrm{N}, 102^{\circ} 09^{\prime} 07^{\prime \prime} \mathrm{W}$, Sierra-Muñoz 677 (HUAA); 4 km al E de Tepezalá, $22^{\circ} 13^{\prime} 13^{\prime \prime} \mathrm{N}, 102^{\circ} 08^{\prime} 06^{\prime \prime} \mathrm{W}$, Sierra-Muñoz 688 (HUAA); Carr. Tepezalá-Puerto de la Concepción a 3 km al SE de Tepezalá, 22 $2^{\circ} 12^{\prime} 30^{\prime \prime} \mathrm{N}, 102^{\circ} 08^{\prime} 24^{\prime \prime} \mathrm{W}$, Sierra-Muñoz 763 (HUAA); Cañada Arroyo Hondo, $1.2 \mathrm{~km}$ al W de Arroyo Hondo, $22^{\circ} 11^{\prime} 16^{\prime \prime} \mathrm{N}, 102^{\circ} 12^{\prime} 09^{\prime \prime} \mathrm{W}, \mathrm{M}$. E. Mendoza 650 (HUAA); Arroyo Las Pilas, $2 \mathrm{~km}$ al NW de la exhacienda Las Pilas, $22^{\circ} 15^{\prime} 59^{\prime \prime} \mathrm{N}$, $102^{\circ} 10^{\prime} 50^{\prime \prime} \mathrm{W}$, M. E. Mendoza 644 (HUAA); Arroyo Las Pilas, $1.3 \mathrm{~km}$ al NW de la exhacienda Las Pilas, $22^{\circ} 15^{\prime} 43^{\prime \prime} \mathrm{N}, 102^{\circ} 10^{\prime} 32^{\prime \prime} \mathrm{W}$, M. E. Mendoza 634 (HUAA). Solanum erianthum. Mpio. Calvillo: Cañada del Temazcal, $0.5 \mathrm{~km}$ al S de presa El Temazcal,

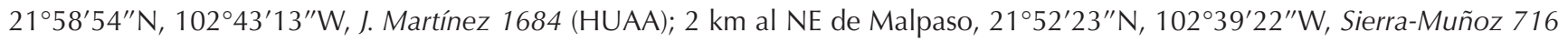
(HUAA); Alrededores de la presa Malpaso, $2^{\circ} 51^{\prime} 07^{\prime \prime} \mathrm{N}, 102^{\circ} 38^{\prime} 56^{\prime \prime} \mathrm{W}$, Sierra-Muñoz 723 (HUAA). Solanum ferrugineum. Mpio.

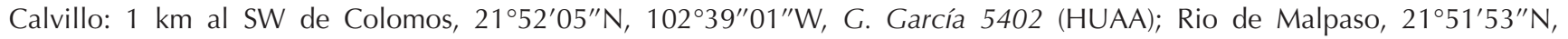

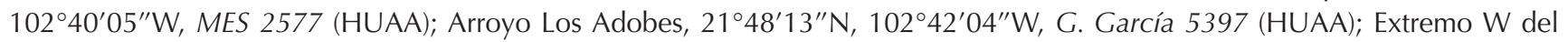
Tepozán, 21 $1^{\circ} 56^{\prime} 20^{\prime \prime} \mathrm{N}, 102^{\circ} 44^{\prime} 13^{\prime \prime} \mathrm{W}$, De la Cerda-G. García 1307 (HUAA); Arroyo Las Moras al N de la Presa Las Moras,

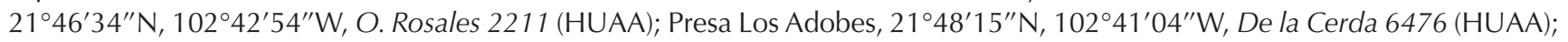
Barranca Las Moras, $1 \mathrm{~km}$ al SE de la cortina de la presa Las Moras, $21^{\circ} 46^{\prime} 06^{\prime \prime} \mathrm{N}, 102^{\circ} 42^{\prime} 52^{\prime \prime} \mathrm{W}$, Sierra-Muñoz 627 (HUAA); $1 \mathrm{~km}$ 
Solanaceae en Aguascalientes, México

Apéndice I. Continuación.

al SE de Mesa Grande, $21^{\circ} 46^{\prime} 40^{\prime \prime} \mathrm{N}, 102^{\circ} 42^{\prime} 56^{\prime \prime} \mathrm{W}$, Sierra-Muñoz 635 (HUAA); Parte media de la barranca La Botita, 4 km al SE

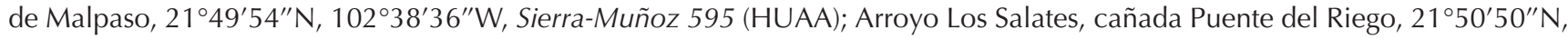

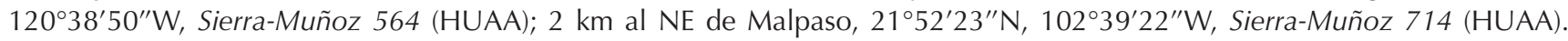
Solanum grayi var. grandiflorum. Mpio. Calvillo: $3 \mathrm{~km}$ al SW del Sauz, 21 $52^{\prime} 31^{\prime \prime} \mathrm{N}, 102^{\circ} 38^{\prime} 41^{\prime \prime} \mathrm{W}$, Sierra-Muñoz 505 (HUAA);

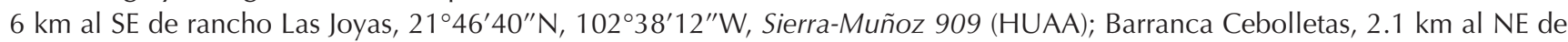

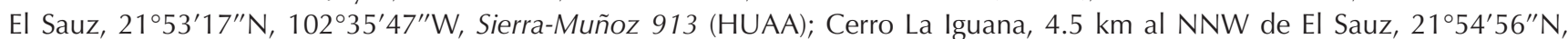
102 38'36"W, Sierra-Muñoz 916 (HUAA); 2.7 km al SW de Presa de los Serna, barranca 3 Cruces, G. García 4751 (IEB). Solanum

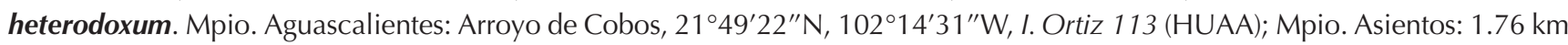
al SE de El Tule, $22^{\circ} 01^{\prime} 58^{\prime \prime} \mathrm{N}, 102^{\circ} 05^{\prime} 51^{\prime \prime} \mathrm{W}, \mathrm{M}$. E. Mendoza 762 (HUAA); Cosío: $2 \mathrm{~km}$ al SW de Cosío, 22 $2^{\circ} 21^{\prime} 13^{\prime \prime} \mathrm{N}, 102^{\circ} 18^{\prime} 56^{\prime \prime} \mathrm{W}$, J. Martínez 2268 (HUAA). Solanum houstonii. Mpio. Calvillo: 2 km al SE de Jaltiche de Arriba, cañada El Tambor, G. García 5289 (HUAA). Solanum lycopersicum. Mpio. Calvillo: 500 m al W de Colomos, 2152'52”N, 102³9’15”W, G. García 5577 (HUAA);

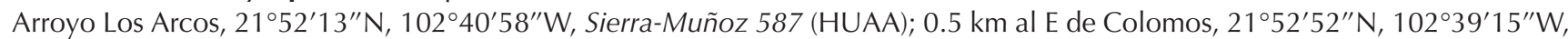
J. Martínez 1354 (INEGI). Solanum nigrescens. Mpio. Aguascalientes: Cerro de Los Gallos, 2139'32”N, 102 ${ }^{\circ} 13^{\prime} 59^{\prime \prime} \mathrm{W}, \mathrm{O}^{\circ}$. Rosa-

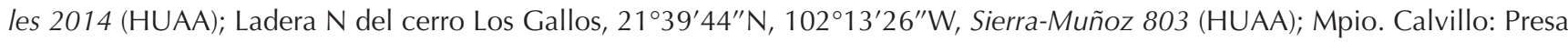
Los Alamitos, límite con Jalisco, Sierra del Laurel, $21^{\circ} 43^{\prime} 58^{\prime \prime} \mathrm{N}, 102^{\circ} 42^{\prime} 33^{\prime \prime} \mathrm{W}$, O. Rosales 3813 (HUAA); 1 km al SE de la entra-

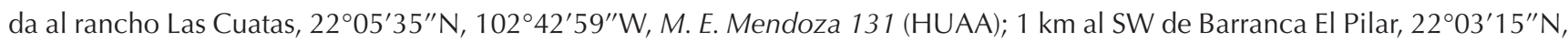
$102^{\circ} 44^{\prime} 07^{\prime \prime} \mathrm{W}$, M. E. Mendoza 149 (HUAA); A $2.3 \mathrm{~km}$ al NE de presa Los Alamitos, $21^{\circ} 44^{\prime} 40^{\prime \prime} \mathrm{N}, 102^{\circ} 41^{\prime} 09^{\prime \prime} \mathrm{W}$, M. E. Mendoza

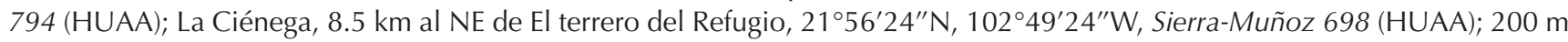
al E de la cortina de la presa Los Alamitos, $21^{\circ} 43^{\prime} 46^{\prime \prime} \mathrm{N}, 102^{\circ} 42^{\prime} 44^{\prime \prime} \mathrm{W}$, Sierra-Muñoz 502 (HUAA); 2 km al NE de El Gatuño,

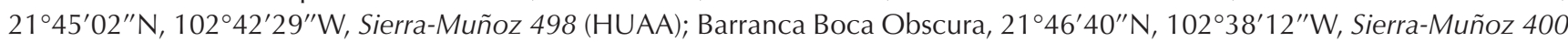
(HUAA); Mpio. El Llano: Barranca de La Mina, $6.7 \mathrm{~km}$ al NE de Palo Alto, 21 ${ }^{\circ} 56^{\prime} 34^{\prime \prime} \mathrm{N}, 101^{\circ} 54^{\prime} 23^{\prime \prime} \mathrm{W}$, M. E. Mendoza 612

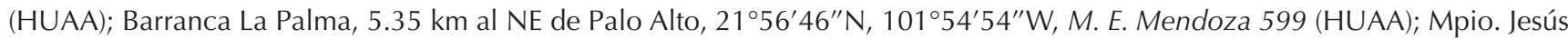

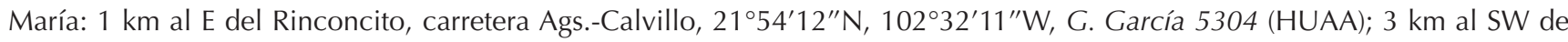

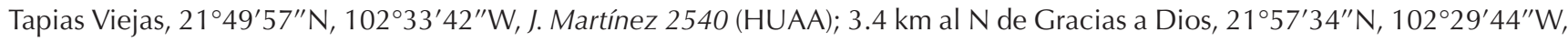
M. E. Mendoza 700 (HUAA); Mpio. Rincón de Romos: 3 km al SW de La Punta, 22 ${ }^{\circ} 18^{\prime} 39^{\prime \prime} \mathrm{N}, 102^{\circ} 19^{\prime} 11^{\prime \prime} \mathrm{W}$, Sierra-Muñoz 846

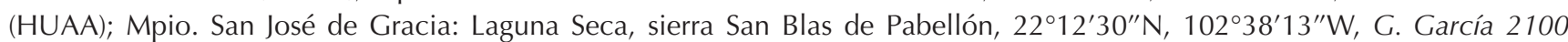
(HUAA; IEB); Cañada a $1.2 \mathrm{~km}$ al SE de Potrero de Los López, 22 ${ }^{\circ} 04^{\prime} 51^{\prime \prime} \mathrm{N}, 102^{\circ} 30^{\prime} 53^{\prime \prime} \mathrm{W}$, J. Martínez 2445 (HUAA); $600 \mathrm{~m}$ al

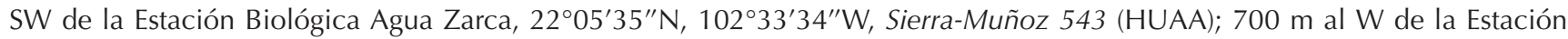

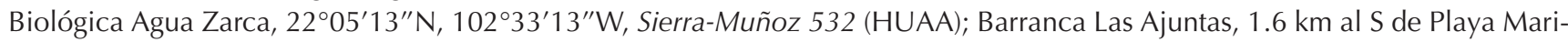

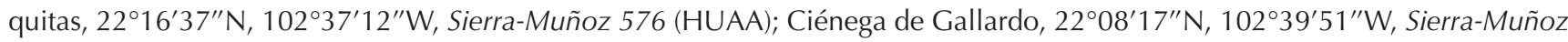
823 (HUAA); 1 km al E de Ciénega de Gallardo, 22 $08^{\prime} 06^{\prime \prime} \mathrm{N}, 102^{\circ} 39^{\prime} 24^{\prime \prime} \mathrm{W}$, Sierra-Muñoz 826 (HUAA); Laguna Seca a 6.9 km al W de La Congoja, $22^{\circ} 10^{\prime} 02^{\prime \prime} \mathrm{N}, 102^{\circ} 37^{\prime} 36^{\prime \prime} \mathrm{W}$, Sierra-Muñoz 888 (HUAA); Rancho Antrialgo, Barranca Juan Francisco a 4.6 km

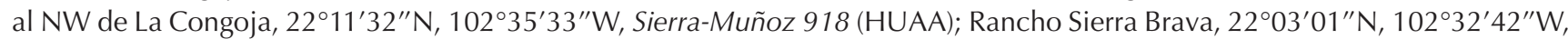
M. E. Mendoza 755 (HUAA). Solanum polyadenium. Mpio. Calvillo: $3.5 \mathrm{~km}$ al NW de Mesa del Roble, G. Murillo 496 (HUAA).

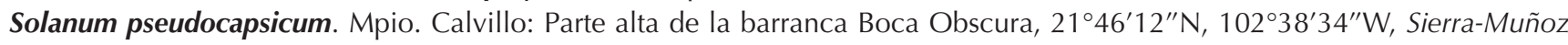
296 (HUAA); Barranca Las Moras, 1 km al SE de la cortina de la presa Las Moras, $21^{\circ} 46^{\prime} 06^{\prime \prime} \mathrm{N}, 102^{\circ} 42^{\prime} 52^{\prime \prime} \mathrm{W}$, Sierra-Muñoz 630 (HUAA); 7 km al NE de Jaltiche de Arriba, 21 ${ }^{\circ} 45^{\prime} 05^{\prime \prime} \mathrm{N}, 102^{\circ} 45^{\prime} 02^{\prime \prime} \mathrm{W}$, Sierra-Muñoz 493 (HUAA); Arroyo Los Salates, cañada Puente del Riego, $21^{\circ} 50^{\prime} 50^{\prime \prime} \mathrm{N}, 102^{\circ} 38^{\prime} 50^{\prime \prime} \mathrm{W}$, Sierra-Muñoz 564 (HUAA). Solanum rostratum. Mpio. Aguascalientes: 2 km al W de San Francisco de los Viveros, $2^{\circ} 54^{\prime} 44^{\prime \prime} \mathrm{N}, 102^{\circ} 06^{\prime} 29^{\prime \prime} \mathrm{W}$, De la Cerda 4988 (HUAA); Ojo de Palmitas, 1.5 km al E de Ags.,

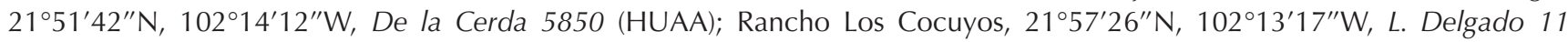
(HUAA); $1.5 \mathrm{~km}$ al NE de la CFE, carretera a San Bartolo, 21 ${ }^{\circ} 45^{\prime} 38^{\prime \prime} \mathrm{N}, 102^{\circ} 11^{\prime} 51^{\prime \prime} \mathrm{W}$, Sierra-Muñoz 470 (HUAA); 9 km al SSE del cruce carretera 45 a San Bartolo, $21^{\circ} 43^{\prime} 50^{\prime \prime} \mathrm{N}, 102^{\circ} 11^{\prime} 06^{\prime \prime} \mathrm{W}$, Sierra-Muñoz 479 (HUAA); 3 km al NE de Los Caños,

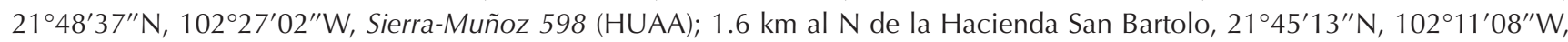
Sierra-Muñoz 812 (HUAA); 1 km al SE de Tanque de Los Jiménez, 21 ${ }^{\circ} 42^{\prime} 03^{\prime \prime} \mathrm{N}, 102^{\circ} 21^{\prime} 57^{\prime \prime} \mathrm{W}$, Sierra-Muñoz 870 (HUAA); 6.5 km

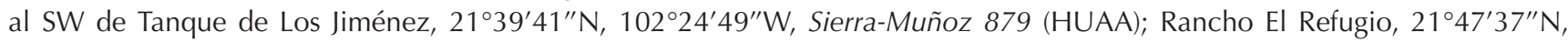
$102^{\circ} 15^{\prime} 10^{\prime \prime} \mathrm{W}$, Sierra-Muñoz 900 (HUAA); 4.27 km al SE de Villa Lic. Jesús Terán, $21^{\circ} 47^{\prime} 08^{\prime \prime} \mathrm{N}, 102^{\circ} 12^{\prime} 06^{\prime \prime} \mathrm{W}$, Sierra-Muñoz 902

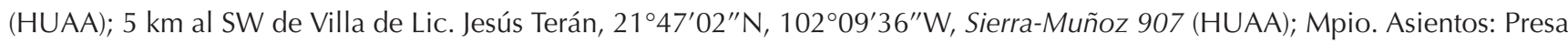
Los Alcantares, Villa Juárez, $22^{\circ} 06^{\prime} 16^{\prime \prime} \mathrm{N}, 102^{\circ} 02^{\prime} 31^{\prime \prime} \mathrm{W}$, P. García 35 (HUAA); Bordo al extremo SW de Plutarco Elías Calles,

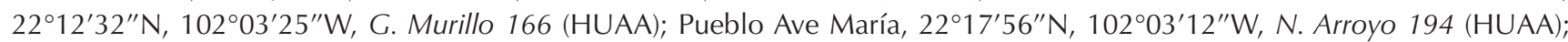

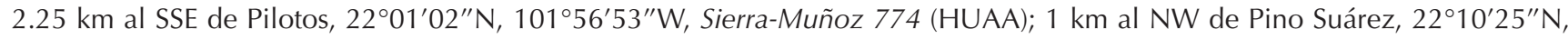
$102^{\circ} 06^{\prime} 14^{\prime \prime}$ W, M. E. Mendoza 769 (HUAA); Mpio. Calvillo: Cañada Cebolletas, $21^{\circ} 52^{\prime} 45^{\prime \prime} \mathrm{N}, 102^{\circ} 37^{\prime} 24^{\prime \prime}$ W, G. Nieto 24 (HUAA); 
José CARlos Sierra-Muñoz et AL.

Apéndice I. Continuación.

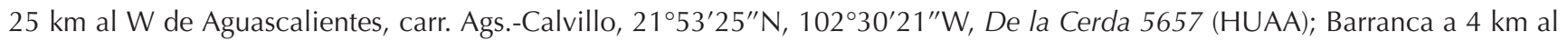

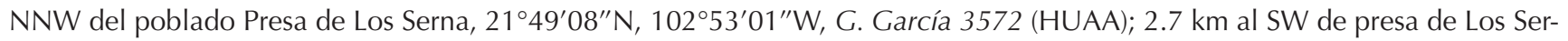

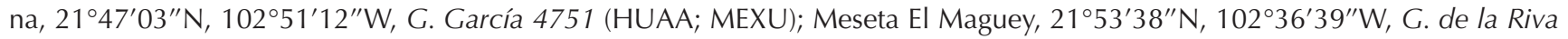

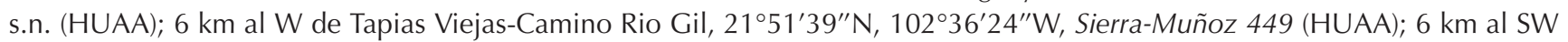

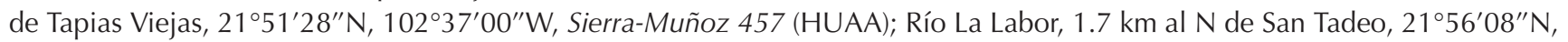
$102^{\circ} 42^{\prime} 06^{\prime \prime} \mathrm{W}$, G. Murillo 324 (HUAA); $950 \mathrm{~m}$ al NE de El Terrero de la Labor, $22^{\circ} 01^{\prime} 50^{\prime \prime} \mathrm{N}, 102^{\circ} 39^{\prime} 50^{\prime \prime} \mathrm{W}, \mathrm{I}$. Ortiz 52 (HUAA);

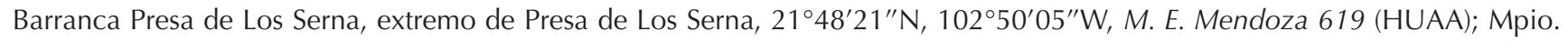

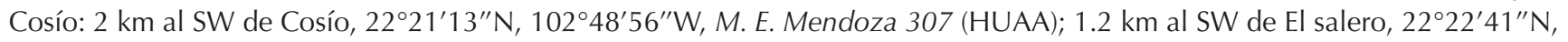
$102^{\circ} 20^{\prime} 03^{\prime \prime} \mathrm{W}$, M. E. Mendoza 714 (HUAA); Mpio. El Llano: $500 \mathrm{~m}$ al N de Las Flores, $21^{\circ} 54^{\prime} 44^{\prime \prime} \mathrm{N}, 102^{\circ} 07^{\prime} 12^{\prime \prime} \mathrm{W}$, De la Cerda

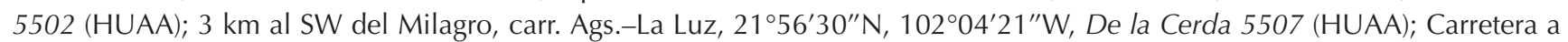

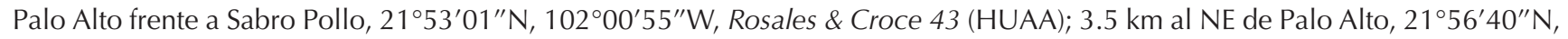
$101^{\circ} 56^{\prime} 45^{\prime \prime} \mathrm{W}$, Sierra-Muñoz 759 (HUAA); Mpio. Jesús María: San Isidro, carr. Calvillo - Tapias Viejas, 22 $2^{\circ} 01^{\prime} 12^{\prime \prime} \mathrm{N}, 102^{\circ} 08^{\prime} 07^{\prime \prime} \mathrm{W}$,

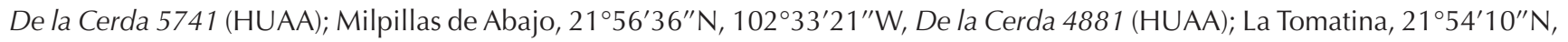

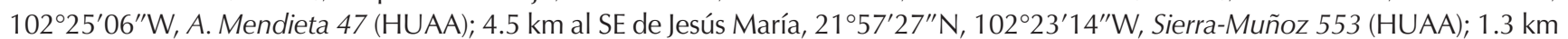

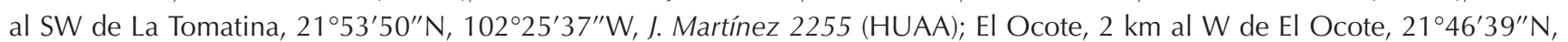

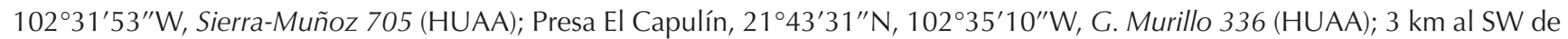

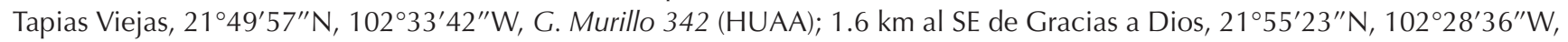

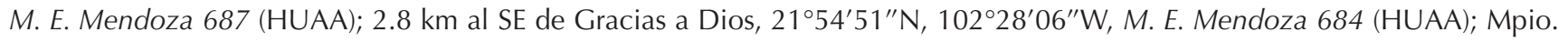
Pabellón de Arteaga: Norte de Ojo Zarco, carr. a Asientos, $22^{\circ} 08^{\prime} 01^{\prime \prime} \mathrm{N}, 102^{\circ} 15^{\prime} 19^{\prime \prime} \mathrm{W}$, De la Cerda 5933 (HUAA); $1.5 \mathrm{~km}$ del entronque Pabellón de Arteaga-Rincón de Romos, $22^{\circ} 11^{\prime} 46^{\prime \prime} \mathrm{N}, 102^{\circ} 18^{\prime} 44^{\prime \prime} \mathrm{W}$, De la Cerda 5689 (HUAA; IEB); Alrededores de la presa El Garabato, $22^{\circ} 05^{\prime} 05^{\prime \prime} \mathrm{N}, 102^{\circ} 20^{\prime} 41^{\prime \prime} \mathrm{W}, \mathrm{M}$. E. Mendoza 589 (HUAA); Mpio. Rincón de Romos: El Salitrillo, 22 ${ }^{\circ} 11^{\prime} 28^{\prime \prime} \mathrm{N}$, $102^{\circ} 17^{\prime} 46^{\prime \prime} \mathrm{W}$, De la Cerda 5493 (HUAA); Extremo NW de la Estancia de Mosqueira, 22 $08^{\prime} 42^{\prime \prime} \mathrm{N}, 102^{\circ} 21^{\prime} 28^{\prime \prime} \mathrm{W}$, Sierra-Muñoz

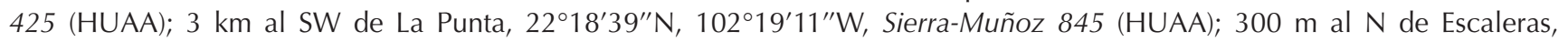
$22^{\circ} 15^{\prime} 26^{\prime \prime} \mathrm{N}, 102^{\circ} 19^{\prime} 54^{\prime \prime} \mathrm{W}$, Sierra-Muñoz 865 (HUAA); Arroyo La Boquilla, $500 \mathrm{~m}$ al W de La Boquilla, 22 $2^{\circ} 15^{\prime} 50^{\prime \prime} \mathrm{N}$, $102^{\circ} 22^{\prime} 37^{\prime \prime} \mathrm{W}$, M. E. Mendoza 577 (HUAA); Mpio. San Francisco de los Romo: 2 km al E de Borrotes, 22 $02^{\prime} 04^{\prime \prime} \mathrm{N}, 102^{\circ} 11^{\prime} 14^{\prime \prime} \mathrm{W}$, Sierra-Muñoz 794 (HUAA); Mpio. San José de Gracia: 6.8 km al NNE de San Antonio de Los Ríos, 22 $2^{\circ} 10^{\prime} 44^{\prime \prime} \mathrm{N}, 102^{\circ} 29^{\prime} 09^{\prime \prime} \mathrm{W}$, De la Cerda 5134 (HUAA); Barranca Revienta Cuartas, Rancho Sierra Hermosa, 22 $10^{\prime} 21^{\prime \prime} \mathrm{N}, 102^{\circ} 36^{\prime} 43^{\prime \prime} \mathrm{W}$, G. Murillo 205 (HUAA); 3 km al N de San José de Gracia, carretera a La Congoja, 22 ${ }^{\circ} 10^{\prime} 25^{\prime \prime} \mathrm{N}, 102^{\circ} 25^{\prime} 10^{\prime \prime} \mathrm{W}$, Sierra-Muñoz 726 (HUAA); Boca de Túnel de Potrerillo, 22 ${ }^{\circ} 14^{\prime} 34^{\prime \prime} \mathrm{N}, 102^{\circ} 26^{\prime} 43^{\prime \prime} \mathrm{W}$, Sierra-Muñoz 730 (HUAA); Cortina de la presa 50 Aniversario, $22^{\circ} 11^{\prime} 04^{\prime \prime} \mathrm{N}$, $102^{\circ} 27^{\prime} 53^{\prime \prime} \mathrm{W}$, Sierra-Muñoz 746 (HUAA); $2.3 \mathrm{~km}$ al NE de rancho Sierra Brava, 22 $03^{\prime} 40^{\prime \prime} \mathrm{N}, 102^{\circ} 32^{\prime} 26^{\prime \prime} \mathrm{W}, \mathrm{M}$. E. Mendoza 758 (HUAA); Rancho Sierra Brava, 22 $03^{\prime} 01^{\prime \prime} \mathrm{N}, 102^{\circ} 32^{\prime} 42^{\prime \prime} \mathrm{W}, \mathrm{M}$. E. Mendoza 754 (HUAA); Mpio. Tepezalá: El Carmen, 4.5 km al W de Tepezalá, $22^{\circ} 13^{\prime} 57^{\prime \prime} \mathrm{N}, 102^{\circ} 11^{\prime} 13^{\prime \prime} \mathrm{W}$, De la Cerda 5978 (HUAA); Presa Mesillas, $1 \mathrm{~km}$ al SE de Mesillas, 22 ${ }^{\circ} 18^{\prime} 43^{\prime \prime} \mathrm{N}$,

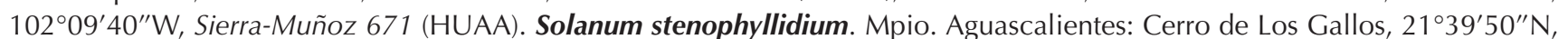

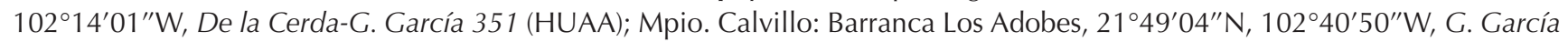
4700 (HUAA); 2 km al E de El Sauz, 21 $51^{\prime} 54^{\prime \prime} \mathrm{N}, 102^{\circ} 36^{\prime} 24^{\prime \prime} \mathrm{W}$, G. García 4629 (HUAA); $6 \mathrm{~km}$ al SE de Jaltiche de Arriba, $21^{\circ} 44^{\prime} 31^{\prime \prime} \mathrm{N}, 102^{\circ} 45^{\prime} 00^{\prime \prime} \mathrm{W}$, Sierra-Muñoz 489 (HUAA); $7 \mathrm{~km}$ al SE de Jaltiche de Arriba, $21^{\circ} 44^{\prime} 31^{\prime \prime} \mathrm{N}, 1^{\circ} 02^{\circ} 45^{\prime} 00^{\prime \prime} \mathrm{W}$, Sierra- $^{-}$ Muñoz 486 (HUAA); 6 km al E del Sauz, 21 $54^{\prime} 21^{\prime \prime} \mathrm{N}, 102^{\circ} 34^{\prime} 57^{\prime \prime} \mathrm{W}$, Sierra-Muñoz 413 (HUAA); Barranca Boca Obscura,

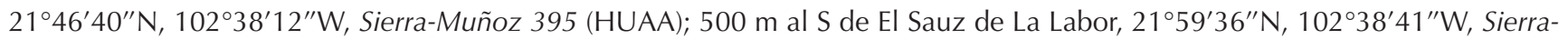

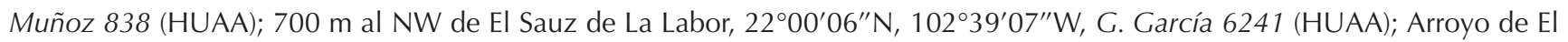
Chivero, $2.2 \mathrm{~km}$ al SE de El terrero del Refugio, $21^{\circ} 51^{\prime} 29^{\prime \prime} \mathrm{N}, 102^{\circ} 49^{\prime} 21^{\prime \prime} \mathrm{W}, \mathrm{M}$. E. Mendoza 628 (HUAA); $1 \mathrm{~km}$ al SE de El Terre-

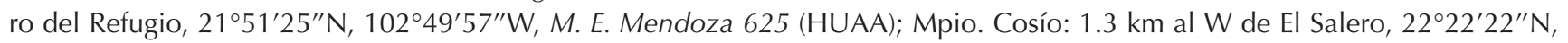
$102^{\circ} 19^{\prime} 27^{\prime \prime} \mathrm{W}$, Rosales \& Croce 115 (HUAA); $1.20 \mathrm{~km}$ al SW de El Salero, 22 $22^{\prime} 41^{\prime \prime} \mathrm{N}, 102^{\circ} 20^{\prime} 03^{\prime \prime} \mathrm{W}$, M. E. Mendoza 711

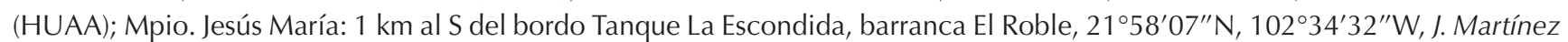

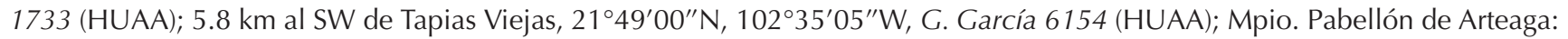

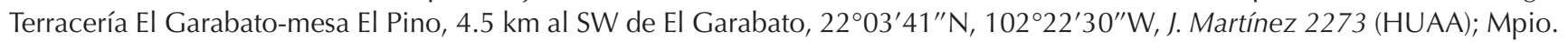

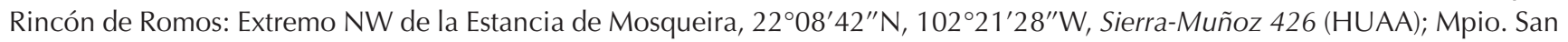

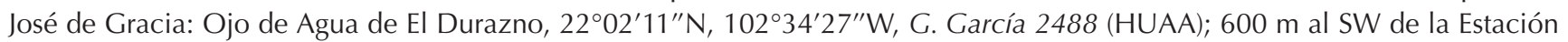

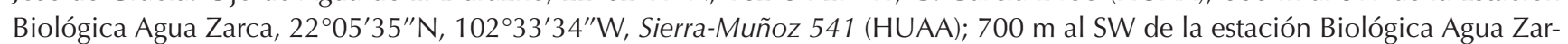

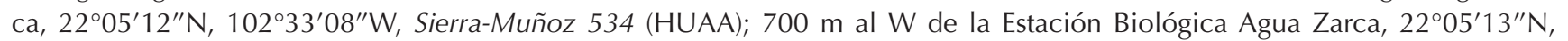

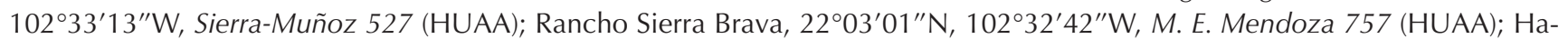
cienda Santa Rosa, $22^{\circ} 01^{\prime} 47^{\prime \prime} N$, 102 $34^{\prime} 22^{\prime \prime}$ W, M. E. Mendoza 744 (HUAA). Solanum stoloniferum. Mpio. Aguascalientes: Ex- 
Solanaceae en Aguascalientes, México

Apéndice I. Continuación.

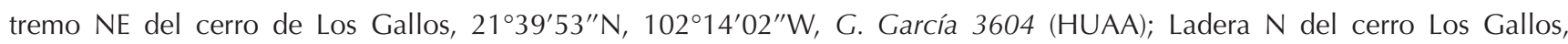
$21^{\circ} 39^{\prime} 44^{\prime \prime} \mathrm{N}, 102^{\circ} 13^{\prime} 26^{\prime \prime} \mathrm{W}$, Sierra-Muñoz 804 (HUAA); Mpio. Calvillo: Sierra el Laurel, near the Jalisco-Aguascalientes border, ca. 10 miles southeast of Calvilo, McVaugh 18359, 18400, 18426 (ENCB); Sur de la presa Los Alamitos, 3 km al E de Los Alisos, $21^{\circ} 43^{\prime} 47^{\prime \prime} \mathrm{N}, 102^{\circ} 42^{\prime} 29^{\prime \prime} \mathrm{W}$, G. García 4335 (HUAA); Presa Los Alamitos, límites con Jalisco, Sierra del Laurel, $21^{\circ} 43^{\prime} 58^{\prime \prime} \mathrm{N}$,

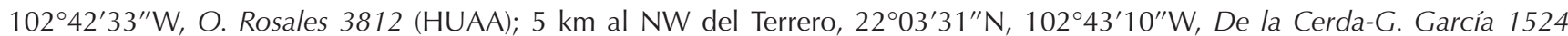
(HUAA); El Pilar, $12 \mathrm{~km}$ al N del Temazcal, 22 ${ }^{\circ} 04^{\prime} 39^{\prime \prime} \mathrm{N}, 102^{\circ} 42^{\prime} 57^{\prime \prime} \mathrm{W}$, G. García 5034 (HUAA); $1 \mathrm{~km}$ al SE de la entrada al

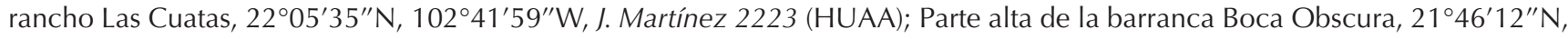

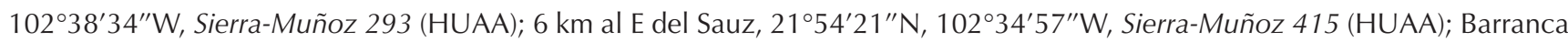

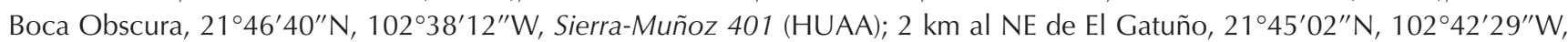

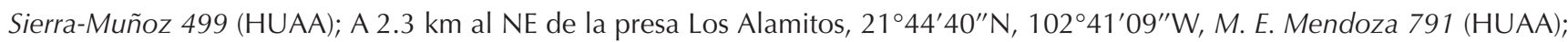

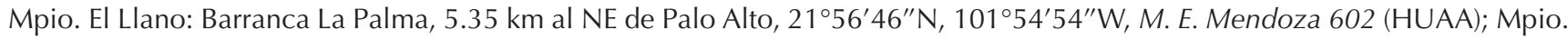

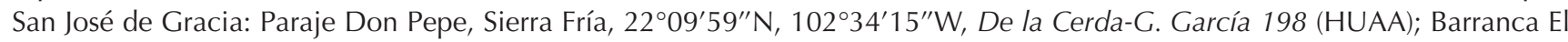

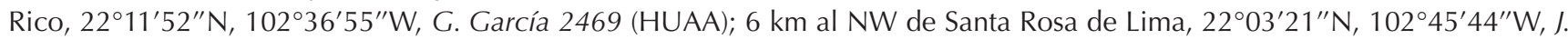
Martínez 1783 (HUAA); Ciénega de Gallardo, 22 $08^{\prime} 17^{\prime \prime} \mathrm{N}, 102^{\circ} 39^{\prime} 51^{\prime \prime} \mathrm{W}$, Sierra-Muñoz 819 (HUAA); 1 km al E de Ciénega de Gallardo, $22^{\circ} 08^{\prime} 06^{\prime \prime} \mathrm{N}, 102^{\circ} 39^{\prime} 24^{\prime \prime} \mathrm{W}$, Sierra-Muñoz 825 (HUAA); Entrada al Zepo a $4.3 \mathrm{~km}$ al NW de La Congoja, 22 ${ }^{\circ} 10^{\prime} 52^{\prime \prime} \mathrm{N}$, $102^{\circ} 35^{\prime} 49^{\prime \prime} \mathrm{W}$, Sierra-Muñoz 844 (HUAA); Laguna Seca a $6.9 \mathrm{~km}$ al W de La Congoja, 22 ${ }^{\circ} 10^{\prime} 02^{\prime \prime} \mathrm{N}, 102^{\circ} 37^{\prime} 36^{\prime \prime} \mathrm{W}$, Sierra-Muñoz 887 (HUAA); 1 km al NW de La Congoja, 22 ${ }^{\circ} 10^{\prime 2} 3^{\prime \prime} \mathrm{N}, 102^{\circ} 33^{\prime} 56^{\prime \prime} \mathrm{W}$, Sierra-Muñoz 891 (HUAA); 3.6 km al NW de La Congoja, $22^{\circ} 11^{\prime} 41^{\prime \prime} \mathrm{N}, 102^{\circ} 34^{\prime} 32^{\prime \prime} \mathrm{W}$, Sierra-Muñoz 895 (HUAA); Arroyo La Mesa del Amo, entre cerro La Calavera y cerro El Colorín, $21^{\circ} 58^{\prime} 32^{\prime \prime} \mathrm{N}, 102^{\circ} 30^{\prime} 56^{\prime \prime} \mathrm{W}$, Sierra-Muñoz 334 (HUAA); Hacienda Santa Rosa, 22 $01^{\prime} 47^{\prime \prime} \mathrm{N}, 102^{\circ} 34^{\prime} 22^{\prime \prime} \mathrm{W}$, M. E. Mendoza 743

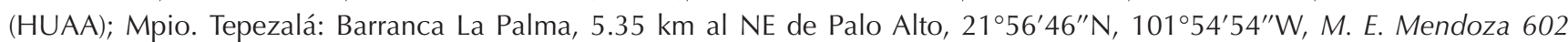
(HUAA); Arroyo Las Pilas, $1.3 \mathrm{~km}$ al NW de la exhacienda Las Pilas, $22^{\circ} 15^{\prime} 43^{\prime \prime} \mathrm{N}, 102^{\circ} 10^{\prime} 32^{\prime \prime} \mathrm{W}$, M. E. Mendoza 636 (HUAA). Solanum trifidum. Mpio. Calvillo: El Salto, Sierra del Laurel, G. García 3687 (HUAA); Mesa El Ocote, 3 km al NW de El Terrero

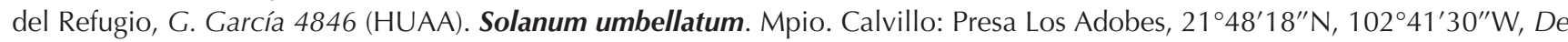

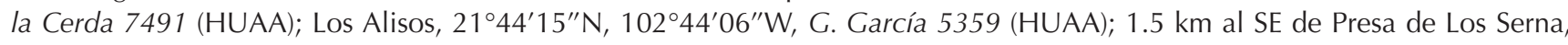

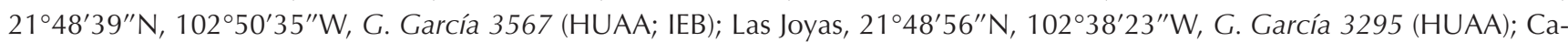
ñada El Guencho, $21^{\circ} 45^{\prime} 21^{\prime \prime} \mathrm{N}, 102^{\circ} 46^{\prime} 19^{\prime \prime} \mathrm{W}$, O. Rosales 3841 (HUAA); Rancho Las Joyas, Sierra del Laurel, $21^{\circ} 47^{\prime} 22^{\prime \prime} \mathrm{N}$, 102 $39^{\prime} 11^{\prime \prime} \mathrm{W}$, J. Martínez 1239 (HUAA; IEB); $6 \mathrm{~km}$ al SE de Jaltiche de Arriba, $21^{\circ} 44^{\prime} 31^{\prime \prime} \mathrm{N}, 102^{\circ} 45^{\prime} 00^{\prime \prime} \mathrm{W}$, Sierra-Muñoz 488

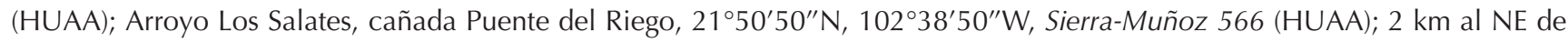

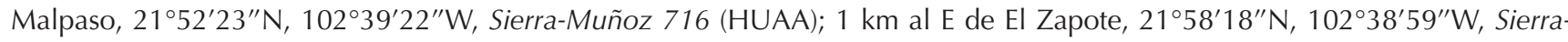
Muñoz 839 (HUAA); A 2.3 km al NW de Mesa del Roble, 214ㄴ $45^{\prime \prime} \mathrm{N}, 102^{\circ} 44^{\prime} 20^{\prime \prime} \mathrm{W}$, G. Murillo 490 (HUAA). 